\title{
Speech meets script - fMRI studies on the integration of letter and speech sounds
}

Citation for published version (APA):

van Atteveldt, N. M. (2006). Speech meets script - fMRI studies on the integration of letter and speech sounds. [Doctoral Thesis, Maastricht University]. Datawyse / Universitaire Pers Maastricht. https://doi.org/10.26481/dis.20060630na

Document status and date:

Published: 01/01/2006

DOI:

10.26481/dis.20060630na

Document Version:

Publisher's PDF, also known as Version of record

\section{Please check the document version of this publication:}

- A submitted manuscript is the version of the article upon submission and before peer-review. There can be important differences between the submitted version and the official published version of record.

People interested in the research are advised to contact the author for the final version of the publication, or visit the DOI to the publisher's website.

- The final author version and the galley proof are versions of the publication after peer review.

- The final published version features the final layout of the paper including the volume, issue and page numbers.

Link to publication

\footnotetext{
General rights rights.

- You may freely distribute the URL identifying the publication in the public portal. please follow below link for the End User Agreement:

www.umlib.nl/taverne-license

Take down policy

If you believe that this document breaches copyright please contact us at:

repository@maastrichtuniversity.nl

providing details and we will investigate your claim.
}

Copyright and moral rights for the publications made accessible in the public portal are retained by the authors and/or other copyright owners and it is a condition of accessing publications that users recognise and abide by the legal requirements associated with these

- Users may download and print one copy of any publication from the public portal for the purpose of private study or research.

- You may not further distribute the material or use it for any profit-making activity or commercial gain

If the publication is distributed under the terms of Article $25 \mathrm{fa}$ of the Dutch Copyright Act, indicated by the "Taverne" license above, 


\section{Speech meets script}

fMRI studies on the integration of letters and speech sounds 
ISBN 10: 90-5278-540-6

ISBN 13: 978-90-5278-540-0

Copyright $(2006$ by Nienke van Atteveldt. All rights reserved. 


\section{Spraak ontmoet schrift}

\section{fMRI studies naar de integratie van letters en spraakklanken}

\section{PROEFSCHRIFT}

ter verkrijging van de graad van doctor aan de Universiteit Maastricht, op gezag van de Rector Magnificus, Prof. Mr. G. P. M. F. Mols volgens het besluit van het College van Decanen, in het openbaar te verdedigen op vrijdag 30 juni 2006 om 14.00 uur

$$
\text { door }
$$

Nienke Mariëlla van Atteveldt

Geboren op 23 september 1977 te IJsselstein

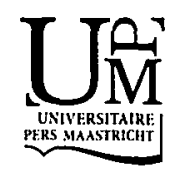


Promotor:

Prof. dr. R. Goebel

Copromotores:

Dr. L. Blomert

Dr. E. Formisano

Beoordelingscommissie:

Prof. dr. G. Kok

(Voorzitter)

Prof. dr. B. M. L. F. de Gelder

(Universiteit van Tilburg)

Prof. dr. P. Hagoort

(F.C. Donders Centre for Cognitive Neuroimaging, Nijmegen)

Dr. B. Jansma

Dr. A. Sack

The research in this dissertation thesis was supported by the Ter Meulen Fund, Royal Netherlands Academy of Arts and Sciences (KNAW) and the Foundation De Drie Lichten in The Netherlands. 
PLOTSELING REALISEERDE IK MIJ HOE COMPLEX DE SITUATIE WAS

Marks en Kampstra, Maastricht

Voor mijn ouders 
CHAPTER 2 Integration of letters and speech sound in the 27 human brain

CHAPTER 3 The effect of temporal asynchrony on the integration of letters and speech sounds

CHAPTER 4 Top-down task effects overrule automatic multisensory responses to letter-sound pairs in auditory association cortex 
Chapter 1

General introduction 
In today's technological information society, the ability to read is essential for social and economic success. Because demands for literacy are constantly increasing, the consequences for those who fail in successful literacy acquisition aggravate accordingly (National Reading Council, 1998; Snowling, 2000). Scientific studies of reading, therefore, form a highly relevant and timely field of research. In alphabetic scripts, such as English or Dutch, letters and speech sounds are the basic elements of correspondence between spoken and written language. Investigations of the mechanism by which letters and speech sounds are associated in the brain are therefore important to increase our understanding of the neural basis of literacy.

In this thesis, a series of fMRI experiments will be presented in which the neural correlates of letter-speech sound association in literate adults were investigated. The first main aim of these experiments was to increase insight in the neural basis of alphabetic reading, by providing a functional neuro-anatomical mechanism for letterspeech sound integration in the normally developed and highly automated system. Therefore, the introduction will start with providing background information on the relation between spoken and written language, to underline the importance of letterspeech sound correspondences in alphabetic scripts.

The second main aim concerns cross-modal information processing. Letter-speech sound correspondences are a special kind of cross-modal associations because they are not naturally, but artificially related and over-learned during reading acquisition and practice. Speech also has a natural visual component: the lip/ mouth movements of the speaker (hereafter referred to as audiovisual speech). Since most research as yet focused on naturally related cross-modal information, the present investigations of the artificially related letters and speech sounds provide novel insights in cross-modal information processing in the human brain. In this introduction, an overview of audiovisual speech integration will be presented as an interesting background for the present experiments on letter-sound integration. Furthermore, different factors that may play a role in efficient letter-sound integration will be discussed. Next, background information will be provided on functional magnetic resonance imaging (fMRI) and finally, the rationale and specific aims of the different experiments in this thesis will be outlined.

\subsection{On the relation between spoken and written language}

It has been said that "reading is parasitic on speech" (Mattingly, 1972), and that learning to read is "an unnatural act" (Gough and Hillinger, 1980). These statements refer to the difference in naturalness between spoken and written language. Spoken language precedes written language, both in phylogenetic and ontogenetic development. In the time course of evolution, spoken language is as old as the human species, while writing systems are cultural inventions that only exist since a few thousands years, and are of common use only since the last century. As a consequence, brain mechanisms for spoken language are a product of biological evolution, while the artificial nature and short history of writing systems make the existence of a naturally adapted specialized brain mechanism for written language unlikely (Gleitman and Rozin, 1977; Liberman, 1992). At the same time, literacy has become extremely important since its short existence. Since the last few decennia, $100 \%$ of the population 
is expected to be literate (National Reading Council, 1998). Considering the artificial nature of written language, meeting this expectation logically poses a serious challenge during individual cognitive development. Before turning to individual development, the origins of writing in human culture will be considered in more detail.

\section{The origins of writing: from rock paintings to the alphabet}

The function of a writing system is to systematically represent spoken language in a less transient form, enabling communication across place and time (Snowling, 2000). The emergence of written language changed communication so drastically that it is impossible to imagine a life today without it; think about newspapers, manuals, textbooks, (e)mail, and so on. The main goal of reading is to extract meaning from written text. The earliest written signs, e.g. rock paintings, were direct expressions of concepts or meanings (semasiography). Since then, various types of script have been invented and used, progressing from meaning-based (logographic) scripts, to the more analytic speech-based (phonographic) alphabetic scripts. In logographic scripts, of which Chinese is the only pure example in current use, symbols represent meaningful units of speech (morphemes or words), with the consequence that many different symbols are needed and have to be memorized (total counts of Chinese logograms approach 90,000). In alphabetic scripts like Dutch or English, symbols represent speech at the more abstract level of single speech sounds (phonemes). The number of symbols in alphabetic scripts (letters of the alphabet) is limited, but at the cost of losing relation to the meanings that have to be conveyed (Gleitman and Rozin, 1977; Liberman, 1992; Treiman and Kessler, 2005). The universality of speech-based writing systems nowadays suggests that they may be more efficient in representing the endless amount of messages that can be conveyed by spoken language (Gleitman and Rozin, 1977; Perfetti and Sandak, 2000).

To sum up, in speech-based alphabetic scripts, spoken and written language are basically related at the level of letters and speech sounds. Because these units are in itself meaningless, this is an abstract level of encoding. Moreover, speech sound units have to be extracted from the continuous speech stream in order to form letter-sound mappings, which is a difficult phonological skill that is not learned automatically (Gleitman and Rozin, 1977).

\section{Literacy acquisition in alphabetic scripts}

As a recapitulation of evolutionary development, spoken language also precedes written language during individual development. Spoken language is acquired automatically and without effort, while literacy is acquired only after a long period of instruction, and is usually learned at an older age (Eden and Moats, 2002), if learned at all. The essential step in becoming literate is to discover how a writing system encodes the already acquired spoken language system. In alphabetic scripts, this is called the alphabetic principle (Byrne et al., 1996). Different developmental theories are in agreement that, although many other factors play a role, learning the letter-sound correspondences of a language is a crucial step in literacy acquisition (Ehri, 2005). Only after mastering the alphabetic principle, all words can be decoded systematically. In this view, learning to read is a cross-modal (audiovisual) task, since visual symbols have to be associated to auditory units of speech (Snowling, 2000). 
As already mentioned, discovering how print encodes speech in alphabetic scripts is difficult and does not occur automatically, because the relevant auditory units are highly abstract and difficult to isolate from the continuous speech stream (Treiman and Kessler, 2005). Especially important for a basic understanding of the alphabetic principle is phoneme awareness: insight that spoken words consist of phonemes, and that letters in written words represent these phonemes (Shaywitz and Shaywitz, 2005; Vellutino et al., 2004). That this insight does not develop automatically during spoken language acquisition is illustrated by studies showing that illiterate adults lack such phonemic awareness (Morais and Kolinsky, 2005).

Letter-sound correspondences seem especially hard to learn and automate in developmental dyslexics (Fox, 1994; Snowling, 1980). Although many different causes have been hypothesized, a consensus has been reached that the core deficit in developmental dyslexia is a phonological processing deficit (Ramus, 2003; Shaywitz and Shaywitz, 2005; Vellutino et al., 2004). Recent electrophysiological studies provide evidence for deviant neural processing of speech sounds in dyslexia (Bonte and Blomert, 2004; Schulte-Korne et al., 2001). A crucial question is how a phonological processing deficit leads to a reading impairment. An often suggested hypothesis is that the phonological deficit impairs the ability to segment spoken words into phonemes, and therefore to link letters to the corresponding phonemes (see e.g. Shaywitz and Shaywitz, 2005; Vellutino et al., 2004). Although this is highly likely, it has hardly ever been tested empirically. The recently suggested hypothesis of allophonic speech perception in dyslexia (Serniclaes et al., 2004) directly predicts consequences for letter-sound mappings that can be tested empirically. This hypothesis is based on the finding that dyslexics show worse discrimination between but better discrimination within phoneme categories (Serniclaes et al., 2001). In other words, dyslexics are more sensitive to different variants of the same phoneme (allophones), which is likely to disturb the formation of the categorical mappings between phonemes and letters (Blomert, 2005).

More support for the importance of mastering letter-sound correspondences for adequate reading achievements is provided by the results of various training strategies and intervention studies. It has been shown that intervention programs that train phonemic awareness are able to improve reading ability (Torgesen, 2005; Vellutino et al., 2004), but are most effective if they include explicit training of letter-sound correspondences (Bus and van IJzendoorn, 1999; Foorman et al., 1998; Simos et al., 2002).

Considering the artificial and abstract nature of alphabetic writing systems, and the phonological skills needed before literacy can be acquired successfully, it is quite surprising that the majority of children learn to read with relative ease (Snowling, 2000). For example, in The Netherlands, the prevalence rate of developmental dyslexia among school children has recently been estimated to be only $3.6 \%$. Moreover, $90 \%$ of school children learn the letter-sound correspondences of Dutch within a few months (Blomert, 2005). These achievements seem remarkable given that our brains are not evolutionarily adapted to process written language. An interesting possible explanation is that the association of written and spoken language may benefit from the phylogenetically older brain mechanism for integrating speech with the corresponding lip/ mouth movements of the speaker. 


\section{Letters and speech sounds form the basis of literacy skills}

We have seen that learning the correspondences between letters and speech sounds is an essential step in literacy acquisition. Skilled reading most probably still involves automatic activation of phonological representations (National Reading Council, 1998; Perfetti and Sandak, 2000; Snowling and Hulme, 2005). This means that after successful acquisition of the alphabetic principle and many years of reading experience, letter-speech sound associations are over-learned paired associates in literate adults. In the present thesis, the neural integration of letters and speech sounds was investigated in literate adults, to increase our understanding of the normally developed, highly automated system. This may serve as a basis for further research aimed at elucidating the development and automation of letter-sound mappings.

It should be noted that the terms graphemes and phonemes are often used to indicate the smallest units of the written and spoken language systems, graphemes being the written representations of phonemes. However, there is no consensus on how exactly phonemes are represented by graphemes. This may, for example, depend on the characteristics of a given language (Hanna et al., 1966; Henderson, 1985; Rey et al., 2000). In the experiments reported in this thesis, we used single letters of the Dutch orthography that represent single speech sound units (e.g., letter "a" and sound $/ \mathrm{a} /$; letter " $r$ " and sound $/ \mathrm{r} /$ ). Thus, all used letter-sound pairs are direct mappings between Dutch orthography and phonology at the most basic level. Therefore, the simplified terms letters and speech sounds will be used throughout this thesis.

To conclude, in alphabetic scripts, spoken and written language are basically related at the level of letters and speech sounds. To gain more insight in the neural basis of literacy skills, letter-speech sound associations are the most fundamental level of connections between written and spoken language to study. As mentioned before, associations between letters and speech sounds are cross-modal (audiovisual) associations. To learn and use these cross-modal associations, the brain has to integrate visual and auditory information, as will further be discussed in the next section.

\subsection{The merging of the senses}

In addition to parallel information processing in specialized sensory systems, information provided by the different senses has to be synthesized in the brain to produce a coherent percept of the environment. This "merging of the senses" (Stein and Meredith, 1993) is called cross-modal or multisensory integration. Information from the different senses complements and confirms each other, so cross-modal integration produces richer and more adequate percepts. This improves the reliability of perception and enhances detection, localization and identification of external events. Furthermore, it enables compensation for degraded senses, for example, lip reading improves speech comprehension in noisy environments (Sumby and Pollack, 1954). Next to unifying percepts of the environment, cross-modal integration permits the learning of relations between stimuli in different modalities (McIntosh et al., 1998; Stein and Meredith, 1993). For example, the sound of a barking dog raises the expectancy of seeing the dog. Especially this second function is relevant for the present thesis, since mappings 
between letters and speech sounds are cross-modal associations that have to be learned during reading acquisition.

In the case of a barking dog or a talking person, relations between sensory events in different modalities are defined in a natural way. For example, a talking person simultaneously emits speech sounds and corresponding visible movements of the face (Bertelson and de Gelder, 2004). Alternatively, cross-modal associations can also be related artificially: letters and speech sounds exhibit no natural corresponding features but are related in an arbitrary way. Spoken language is associated with visual information in both ways: naturally with face movements (audiovisual speech), and artificially with written language. While much research has focused on audiovisual speech, the neural mechanism for integrating speech and written language is largely unknown. The mechanism for audiovisual speech integration as revealed by other neuroimaging studies will be presented to serve as a background for the present experiments on letter-speech sound integration. Next, different factors that determine cross-modal integration (binding factors) will be discussed, to provide a background for studying the critical factors in successful integration of letters and speech sounds.

\section{A natural and an artificial visual component of speech}

As mentioned above, speech is associated with a visual component both naturally (audiovisual speech), as well as artificially (written language). At the behavioral level, it has been reported that speech perception can be influenced both by lip movements and by letters. Sumby and Pollack (1954) showed that lip reading can improve speech perception, especially in situations when the auditory input is degraded. More extremely, lip reading can also change the auditory speech percept, as is shown by the McGurk effect (McGurk and MacDonald, 1976). Improvement of speech perception by print has also been demonstrated, at the level of words (Frost et al., 1988), syllables (Massaro et al., 1988), and phonemes (Dijkstra et al., 1989). An interesting next question concerns the neural mechanisms by which speech is associated with both visual components in the brain.

The neural mechanism for audiovisual speech integration has been investigated extensively over the past decade. Results of these neuroimaging studies suggest that the perceptual gain experienced when perceiving multimodal speech is accomplished by enhancement of the neural activity in the relevant sensory cortices. The left posterior superior temporal sulcus (STS) has been advanced as the heteromodal site that integrates visual and auditory speech information, and modulates the modality-specific cortices via back-projections (Calvert et al., 1999; Calvert et al., 2000). This feedback mechanism suggested by Calvert and colleagues was supported by other neuroimaging findings on audiovisual speech perception (Sams et al., 1991; Sekiyama et al., 2003; Wright et al., 2003) and lip reading (Calvert et al., 1997; Calvert and Campbell, 2003; Paulesu et al., 2003). Interestingly, temporal coherence appears to be of limited importance for audiovisual speech signals to be integrated (Massaro and Cohen, 1993; Massaro et al., 1996; Munhall et al., 1996; Munhall and Vatikiotis-Bateson, 2004).

The neural mechanism that mediates the integration of letters and speech sounds is less clear. Raij and colleagues (2000) measured magnetic brain responses to unimodally and bimodally presented letters and speech sounds using magnetoencephalography (MEG). Their findings suggest that the STS is also involved in audiovisual letter integration. Furthermore, the results provide timing information that supports a 
feedback mechanism: convergence and interaction effects started at $225 \mathrm{~ms}$, while differential interaction effects for matching and nonmatching letters and speech sounds did not occur until $380-450 \mathrm{~ms}$. More evidence for the involvement of the STS/STG in letter-sound integration is provided by a recent learning experiment using fMRI (Hashimoto and Sakai, 2004). Learning of new letter-sound mappings in skilled readers involved a network of inferior temporal and parieto-occipital regions, while the STG/MTG was active during processing of already acquired letter-sound correspondences. The fMRI study reported in chapter 2 provides more detailed insight in the exact location and functional role of brain areas involved in letter-sound integration. The results reported in chapter 2 also provide a basis for investigating the role of different factors in the neural integration of letters and speech sounds.

\section{Factors that determine cross-modal integration}

For unraveling how information from different sensory modalities is integrated, a principle question is how the brain knows which information provided by the different senses belongs together. Different binding factors have been demonstrated to play a role in cross-modal integration. These factors can be defined either by features of the stimuli themselves (stimulus-related), or imposed by top-down task instructions (taskrelated). The relative importance of these different binding factors may be weighted differently, depending on the nature and complexity of the relation between the multisensory inputs, and the purpose of integration (Calvert et al., 1998, see also chapter 3).

Stimulus-related binding factors include temporal and spatial correspondence and shared information content (meaning/identity information). In the natural environment, multimodal inputs provide temporally and spatially coincident information about the same event. Spatial and temporal correspondence are therefore key determinants for cross-modal binding, which is demonstrated for multisensory neurons in the superior colliculus and cortex in the cat (Meredith et al., 1987; Stein and Wallace, 1996) as well as in primates (Wallace et al., 1996). In these studies, simple transient stimuli such as light flashes and noise bursts are commonly used (for a review, see Stein and Meredith, 1993). When the complexity of multisensory information increases, information content of the unisensory inputs may serve as an additional binding factor (Calvert et al., 1998; Laurienti et al., 2004; Pourtois and de Gelder, 2002).

In the case of letters and speech sounds, the visual and auditory signals do not emanate from the same environmental event, but are related artificially. Since inherently corresponding temporal or spatial features are lacking, artificially related information is initially (during the learning process) related exclusively by information content (i.e., meaning/identity). However, the role of different stimulus-related factors in the binding of over-learned and automated artificial associations is unclear. In chapter 3, the relative importance of information content (identity congruency) and temporal proximity in the integration of letters and speech sounds in literate adults was investigated.

Next to stimulus-related factors, task-related factors also have a clear effect on multisensory integration. For example, Anderson and colleagues (2004) showed for identical stimuli that the occurrence and nature of multisensory effects (indicated by illusory fusions and fissions of beeps and flashes) were strongly influenced by different task instructions. The strong task effects indicated that visual and auditory stimuli 
where not necessarily automatically integrated in the same way under different task conditions. More generally speaking, demands of laboratory tasks may contaminate automatic perceptual integration processes and thereby complicate the interpretation of experimental results (de Gelder and Bertelson, 2003). Therefore, it is of high importance to compare integration with and without active task demands. Neurophysiological measures are ideal dependent variables for this purpose, because they allow neural integration to be compared directly between passive (without task demands) integration and active (with task demands) integration of identical stimuli. A comparison of passive and active integration of letters and speech sounds will be reported in chapter 4 .

\section{Letters and speech sounds: neural mechanism and binding factors}

To conclude, ample evidence is already provided for a neural mechanism of audiovisual speech processing relying on integration in the heteromodal STS/STG and back projections to sensory cortices. The mechanism by which the brain associates the artificially related letters and speech sounds is not clear yet, although a few studies suggest the involvement of the STS and support the possibility of a similar feedback mechanism. Furthermore, the role of different stimulus- and task-related factors in the integration of the artificially related, over-learned associations between letters and speech sounds has not yet been investigated.

To elucidate these open issues, the fMRI experiments in this thesis aimed to provide more insight in the neural mechanism of letter-speech sound integration, and the role of different binding factors in this process. In chapter 2 , a functional neuro-anatomical mechanism for letter-speech sound integration will be reported. This proposed mechanism will be used as a basis for investigating the role of different binding factors in letter-sound integration: the effect of temporal asynchrony will be reported in chapter 3; the effect of different stimulus presentation mode and task instructions will be reported in chapter 4. The empirical method used in this thesis is functional Magnetic Resonance Imaging (fMRI). A short introduction to this neuroimaging method will be provided next, followed by how fMRI was used in the present experiments to investigate the issues stated above.

\section{3 fMRI of letter-speech sound integration}

Functional imaging methods map the neuro-anatomical localizations of dynamic brain changes during sensory, motor or cognitive activities (Matthews, 2001). The advent of fMRI revolutionized the study of mental processes, because it provided a non-invasive method to study neural activity simultaneously in the whole brain with millimeter resolution. For a better understanding of the experiments reported in this thesis, a short description will be given of how fMRI measures brain activation.

\section{fMRI: What do we measure?}

Although evidence for the direct coupling of neural activity and the fMRI signal has been provided recently (Logothetis, 2003), fMRI is an indirect measure of neural activity because it measures the hemodynamic response to neural activation, rather than neural activity itself. Neural activity occurs when information is transferred in the 
brain. It comprises energy-requiring electrical (axonal and dendritic current flows) and chemical activities (neurotransmitter release and uptake at synaptic contacts). Most energy is used at synapses. Since energy production in neural tissue depends on oxidative metabolism, increased synaptic activity leads to a greater local demand for oxygen delivery. Neural activity is therefore accompanied by increased local blood flow, whereby the increased delivery of oxygen exceeds the increase in oxygen utilization. This leads to a higher ratio of oxy- to deoxyhemoglobin, which provides the main imaging contrast used in $\mathrm{FMRI}$ : the "blood oxygenation level dependent" or "BOLD" contrast. How is the BOLD contrast generated and used in functional imaging?

In a typical fMRI experiment, a subject is placed in the bore of a magnet generating a static magnetic field ( 3 Tesla in the present experiments, which is 60,000 times the strength of the earth's magnetic field (Bandettini et al., 2000)). Hydrogen protons in water molecules in the subject's body (i.e., in the brain tissue), possess a nuclear spin and therefore behave as magnetic dipoles when they are placed in a static magnetic field. Absorption or emission of energy in the radiofrequency (RF) range can change the energy state of the protons, if applied at the resonance (or Larmor) frequency. During functional imaging, RF pulses are applied to excite protons. When the RF field is switched off, protons relax to their low energy states by emission of RF energy that constitutes the MR signal. This relaxation process is characterized by different time constants, of which the transverse relaxation constant $\mathrm{T} 2 *$ (which reflects the rate of signal decay in the presence of local magnetic field inhomogeneities) is used in BOLD fMRI. Oxy- and deoxyhemoglobin differ in magnetic susceptibility, i.e., in interactions with the local magnetic field. The local magnetic field surrounding the protons will be more distorted by the paramagnetic deoxyhemoglobin than by the diamagnetic oxyhemoglobin, causing $\mathrm{T} 2 *$ to decay faster at higher ratios of deoxygenated hemoglobin, and thereby reducing the measured signal. As neural activity leads to a localized increase in oxygenated blood, the proportion of paramagnetic deoxyhemoglobin is reduced, and the MR signal from the protons in the surrounding brain tissue will be increased (Bandettini et al., 2000; Matthews, 2001).

To determine where in the brain the measured signal changes come from, location is encoded during scanning. The resonance frequency of protons is proportional to the strength of the static magnetic field, which can be used to encode position. By superimposing small magnetic field gradients on the static magnetic field along different directions, the resonance frequency of protons and the phase of the emitted signal are unique to each volume unit (voxel) in the 3D grid that is being scanned. This frequency and phase information can be converted into a spatially informative image by inverse Fourier transformation. The timing of applied gradients and RF pulses is called the pulse sequence. In the present experiments, an echo-planar imaging (EPI) sequence was used, which is the most common fast imaging method for fMRI. With EPI, it is possible to acquire whole-brain scans within a few seconds. This enables a relatively fast sampling of the time-course of MR signal changes during sensory stimulation or performance on cognitive or motor tasks. After appropriate statistical analysis, maps can be created of the dynamic brain changes related tot the probed sensory, motor or cognitive activities (Bandettini et al., 2000; Huettel et al., 2004; Matthews, 2001). 


\section{fMRI in the present experiments}

As discussed in $\$ 1.2$, functional imaging studies of the processing and integration of letters and speech sounds are sparse. In the present thesis, fMRI was used to investigate the functional neuroanatomy of the integration of letters and speech sounds with high spatial accuracy, and the critical factors in successful processing of letter-sound associations. Over the past decade, fMRI has been used more and more to explore where and how the human brain combines what we see, hear, feel, taste and smell (Calvert and Lewis, 2004). However, the optimal way to use fMRI to infer cross-modal integration is a matter of heavy debate (see Beauchamp, 2005 and Laurienti et al., 2005 for recent reviews). Detailed discussions of our methodological approaches in the context of this more general debate will be provided in the following empirical chapters. Here, a simplified summary will be given of the rationale of how we used fMRI to address the current research aims.

In the reported experiments, subjects were presented with letters or speech sounds in isolation (unimodal presentation) and letter-sound pairs (bimodal presentation). Lettersound pairs either had the same (congruent) or a different (incongruent) identity. The fMRI time-series measured during the unimodal and bimodal presentation of letters and speech sounds were analyzed to infer where and how cross-modal integration occurred. Two different, complementary analytical approaches were used. First, we compared the fMRI response evoked by bimodal stimulation to each unimodal condition, to find regions responding stronger to letter-sound pairs than to letters and speech sounds presented alone. If such a region also responded significantly both to letters and to speech sounds presented in isolation, it was a plausible candidate for performing cross-modal integration. Second, we contrasted responses to congruent pairs with the responses to incongruent pairs. If certain brain regions respond differentially to congruent and incongruent letter-sound pairs, we can be sure that the brain has successfully compared the visual and auditory inputs.

Although the exact underlying neuronal computations remain speculative, these two main approaches provide fMRI signatures of letter-speech sound integration. Based on the results yielded by both approaches, we propose a functional neuro-anatomical mechanism for letter-speech sound integration in chapter 2 . In the subsequent experiments, the revealed fMRI signatures of letter-sound integration were used to determine the effects of temporal asynchrony (chapter 3), stimulus presentation mode (chapter 4), and task instruction (chapter 4) on the integration of letters and speech sounds in the relevant brain regions. More specifically, the role of these different factors in letter-sound integration was tested by either disrupting a binding factor (e.g. temporal synchrony in chapter 3 ), or changing a factor drastically (e.g. task demands in chapter 4).

\subsection{Summary: research aims and outline of the thesis}

As stated at the beginning of this general introduction, scientific studies of reading form a highly relevant and timely field of research. However, essential knowledge concerning the neural basis of literacy acquisition is still lacking. Although biological correlates of reading and its development have been studied extensively over the past 
few decades (Price and McCrory, 2005), very few studies focused on the basic elements of literacy acquisition: the correspondences between letters and speech sounds of a language. Investigations of the functional neuro-anatomy and critical factors in successful processing of letter-sound associations is therefore crucial for a better understanding of the neural processes underlying reading ability.

\section{General research aims of the thesis}

The series of experiments in the present thesis address two main aims:

1. To increase our understanding of the neural basis of literacy in alphabetic scripts;

2. To provide novel insights in cross-modal information processing in the human brain.

For these purposes, fMRI was used to elucidate the functional neuro-anatomical mechanism for letter-speech sound integration in the normally developed and highly automated system (chapter 2). This will contribute to a better understanding of the neural basis of alphabetic reading. Furthermore, we used fMRI to investigate the role of different stimulus- and task-related factors in letter-sound integration (chapters 3 and 4). This will determine the critical factors in successful processing of letter-sound associations. All experiments will provide fundamental insights in how the brain integrates artificially associated cross-modal information.

In addition, the results of the fMRI experiments in this thesis will have more indirect, but very relevant implications for future studies. Knowledge about the neural mechanism for letter-speech sound integration in literate adults provides a basis for follow-up research concerning the development and automation of letter-sound associations during literacy acquisition. Furthermore, it may provide a basis to test whether letter-sound mappings develop abnormally in developmental dyslexia. An interesting potential application in the context of developmental dyslexia is that the present experiments may provide a basis to biologically validate training effects. In $\S 1.1$, it was mentioned that training programs are able to improve reading ability, but are most effective if they include letter-sound training. It would be very interesting to find out whether neural letter-sound processing is changed into the normal pattern after a period of training.

\section{Specific research aims addressed in the following chapters}

Chapter 2 investigates the functional neuroanatomy of the integration of letters and speech sounds. In the reported fMRI experiment, subjects passively listened to and/or viewed unimodally or bimodally presented speech sounds and letters; bimodal stimuli were either congruent or incongruent (i.e. same/different identity). Because the stimuli were perceived passively, the results of this study provide a "default" neural mechanism, for the automatic/mandatory integration of simultaneously presented letters and speech sounds.

Chapter 3 investigates how the functional neuroanatomical mechanism of the integration of letters and speech sounds reported in chapter 2 is influenced by temporal asynchrony of the letters and speech sounds. In the reported fMRI experiment, both the temporal relation (stimulus onset asynchrony, SOA) and content congruency (same/different identity) between letters and speech sounds were manipulated within 
the same experimental design. The results extend the default integration mechanism of letters and speech sounds provided by chapter 2, by adding the role of temporal proximity.

Chapter 4 investigates the effects of stimulus presentation mode and task-related factors on the neural mechanism for letter-speech sound integration. In the experiments reported in chapters 2 and 3, subjects were instructed to carefully view/listen to the stimuli without performing any active task. This means that the incoming information is integrated based on stimulus-related properties only, i.e. identity congruency and temporal proximity. However, as we have seen in $\S 1.2$, top-down factors generated by task instructions also seem to have a clear effect on multisensory integration. In the fMRI and behavioral experiments reported in chapter 4, the experimental design was changed from blocked to random stimulus presentation, and an active task was included: subjects were instructed to perform a same/different matching task on lettersound pairs that were presented simultaneously or at different temporal offsets. The results of chapter 4 complete the results of chapters 2 and 3 , by demonstrating the effects of different experimental designs and task demands.

In chapter 5, the findings of the experiments reported in chapters 2-4 and their implications for literacy and cross-modal processing are discussed, as are the more indirect implications. Furthermore, the present fMRI results will be compared to what is reported in the literature on audiovisual speech integration. Finally, the speculative suggestion that the naturally evolved mechanism for audiovisual speech integration could provide a neural foundation for the coupling of written language to speech will be discussed. 


\section{References}

Andersen, T. S., Tiippana, K., and Sams, M. (2004). Factors influencing audiovisual fission and fusion illusions. Cognitive Brain Research 21, 301-308.

Bandettini, P. A., Birn, R. M., and Donahue, K. M. (2000). Functional MRI: background, methodology, limits, and implementation. In Handbook of Psychophysiology, J. T. Cacioppo, L. G. Tassinary, and G. G. Berntson, eds. (Cambridge University Press).

Beauchamp, M. (2005). Statistical criteria in fMRI studies of multisensory integration. Neuroinformatics 3, 93-113.

Bertelson, P., and de Gelder, B. (2004). The psychology of multimodal perception. In Crossmodal space and crossmodal attention, C. Spence, and J. Driver, eds. (Oxford, Oxford University Press).

Blomert, L. (2005). Dyslexie in Nederland - theorie, praktijk en beleid (Dyslexia in The Netherlands - theory, practice and policy). (Amsterdam, Uitgeverij Nieuwezijds).

Bonte, M. L., and Blomert, L. (2004). Developmental dyslexia: ERP correlates of anomalous phonological processing during spoken word recognition. Cognitive Brain Research 21, 360376.

Bus, A. G., and van IJzendoorn, M. H. (1999). Phonological awareness and early reading: a metaanalysis of experimental training studies. Journal of Educational Psychology 91, 403-4l4.

Byrne, B., Fielding-Barnsley, R., and Ashley, L. (1996). What does a child bring to the task of learning to read? A summary of the New England Reading Acquisition Projects. Australian Journal of Psychology 48, 119-123.

Calvert, G. A., Brammer, M. J., Bullmore, E. T., Campbell, R., Iversen, S. D., and David, A. S. (1999). Response amplification in sensory-specific cortices during crossmodal binding. Neuroreport 10, 2619-2623.

Calvert, G. A., Brammer, M. J., and Iversen, S. D. (1998). Crossmodal identification. Trends in Cognitive Sciences 2, 247-253.

Calvert, G. A., Bullmore, E. T., Brammer, M. J., Campbell, R., Williams, S. C., McGuire, P. K., Woodruff, P. W., Iversen, S. D., and David, A. S. (1997). Activation of auditory cortex during silent lipreading. Science 276, 593-596.

Calvert, G. A., and Campbell, R. (2003). Reading Speech from Still and Moving Faces: The Neural Substrates of Visible Speech. Journal of Cognitive Neuroscience 15, 57-71.

Calvert, G. A., Campbell, R., and Brammer, M. J. (2000). Evidence from functional magnetic resonance imaging of crossmodal binding in the human heteromodal cortex. Current Biology 10 , 649-657.

Calvert, G. A., and Lewis, J. W. (2004). Hemodynamic Studies of Audiovisual Interactions. In The Handbook of Multisensory Processes, G. A. Calvert, C. Spence, and B. E. Stein, eds. (Cambridge, MA, The MIT Press).

de Gelder, B., and Bertelson, P. (2003). Multisensory integration, perception and ecological validity. Trends in Cognitive Sciences 7, 460-467.

Dijkstra, A., Schreuder, R., and Frauenfelder, U. H. (1989). Grapheme context effects on phonemic processing. Language and Speech 32, 89-108.

Eden, G. F., and Moats, L. (2002). The role of neuroscience in the remediation of students with dyslexia. Nature Neuroscience 5, 1080-1084.

Ehri, L. C. (2005). Development of sight word reading: phases and findings. In The Science of Reading: A Handbook, M. J. Snowling, and C. Hulme, eds. (Oxford, Blackwell publishing).

Foorman, B. R., Francis, D. J., Fletcher, J. M., Schatschneider, C., and Mehta, P. (1998). The role of instruction in learning to read: preventing reading failure in at-risk children. Journal of Educational Psychology 39, 37-55. 
Fox, E. (1994). Grapheme-phoneme correspondence in dyslexic and matched control readers. British Journal of Psychology 85, 41-53.

Frost, R., Repp, B. H., and Katz, L. (1988). Can speech perception be influenced by simultaneous presentation of print? Journal of Memory and Language 27, 741-755.

Gleitman, L. R., and Rozin, P. (1977). The structure and acquisition of reading l: relations between orthographies and the structure of language. In Towards a psychology of reading: the proceedings of the CUNY conferences, A. Reber, and D. Scarborough, eds. (Hillsdale, N.J, Lawrence Erlbaum Associates).

Gough, P. B., and Hillinger, M. L. (1980). Learning to read: an unnatural act. Bulletin of the Orton Society: an interdisciplinary journal of specific language disability 30, 179-196.

Habib, M. (2003). Rewiring the dyslexic brain. Trends in Cognitive Sciences 7, 2003.

Hairston, W. D., Burdette, J. H., Flowers, D. L., Wood, F. B., and Wallace, M. T. (2005). Altered temporal profile of visual-auditory multisensory interactions in dyslexia. Experimental Brain Research 166, 474-480.

Hanna, P. R., Hanna, J. A., Hodges, R. E., and Rudorf, E. G. (1966). Phoneme-grapheme correspondences as cues for spelling improvement (Washington DC, US Dept. of Health, Education and Welfare).

Hashimoto, R., and Sakai, K. L. (2004). Learning letters in adulthood: direct visualization of cortical plasticity for forming a new link between orthography and phonology. Neuron 42, 311 1-322.

Henderson, L. (1985). On the use of the term 'grapheme'. Language and Cognitive Processes $I, 135-$ 148.

Huettel, S. A., Song, A. W., and McCarthy, G. (2004). Functional magnetic resonance imaging (Sunderland, MA, Sinauer Associates, Inc).

Laurienti, P. J., Kraft, R. A., Maldjian, J. A., Burdette, J. H., and Wallace, M. T. (2004). Semantic congruence is a critical factor in multisensory behavioral performance. Experimental Brain Research $158,405-414$.

Laurienti, P. J., Perrault, T. J., Stanford, T. R., Wallace, M. T., and Stein, B. E. (2005). On the use of superadditivity as a metric for characterizing multisensory integration in functional neuroimaging studies. Experimental Brain Research 1166, 289-297.

Liberman, A. M. (1992). The relation of speech to reading and writing. In Orthography, Phonology, Morphology and Meaning, R. Frost, and L. Katz, eds. (Amsterdam, Elsevier Science Publishers B.V.).

Logothetis, N. K. (2003). The underpinnings of the BOLD functional magnetic resonance imaging signal. The Journal of Neuroscience 23, 3963-3971.

Massaro, D. W., and Cohen, M. M. (1993). Perceiving asynchronous bimodal speech in consonantvowel and vowel syllables. Speech Communication 13, 127-134.

Massaro, D. W., Cohen, M. M., and Smeele, P. M. (1996). Perception of asynchronous and conflicting visual and auditory speech. Journal of the Acoustical Society of America 100, 17771786.

Massaro, D. W., Cohen, M. M., and Thompson, L. A. (1988). Visible language in speech perception: lipreading and reading. Visible Language 22.

Matthews, P. (2001). An introduction to functional magnetic resonance imaging of the brain. In Functional MRI: An introduction to the methods, P. Jezzard, P. M. Matthews, and S. M. Smith, eds. (Oxford, Oxford University Press).

Mattingly, I. G. (1972). Reading, the linguistic process, and linguistic awareness. In Language by ear and by eye: The relationship between speech and reading, J. F. Kavanagh, and I. G. Mattingly, eds. (Cambridge, MA, MIT Press).

McGurk, H., and MacDonald, J. (1976). Hearing lips and seeing voices. Nature 263, 747. 
McIntosh, A. R., Cabeza, R. E., and Lobaugh, N. J. (1998). Analysis of Neural Interactions Explains the Activation of Occipital Cortex by an Auditory Stimulus. Journal of Neurophysiology 80 , 2790-2796.

Meredith, M. A., Nemitz, J. W., and Stein, B. E. (1987). Determinants of multisensory integration in superior colliculus neurons. I. Temporal factors. Journal of Neuroscience 7, 3215-3229.

Morais, J., and Kolinsky, R. (2005). Literacy and cognitive change. In The science of reading - a handbook, M. Snowling, and C. Hulme, eds. (Oxford, Blackwell Publishing).

Munhall, K., Gribble, P., Sacco, L., and Ward, M. (1996). Temporal constraints on the McGurk effect. Perception \& Psychophysics 58, 351-362.

Munhall, K., and Vatikiotis-Bateson, E. (2004). Spatial and Temporal Constraints on Audiovisual Speech Perception. In The Handbook of Multisensory Processes, G. A. Calvert, C. Spence, and B. E. Stein, eds. (Cambridge, MA, The MIT Press), pp. 177-188.

National Reading Council (1998). Preventing reading difficulties in young children (Washington, DC, National Academy Press).

Paulesu, E., Perani, D., Blasi, V., Silani, G., Borghese, N. A., De Giovanni, U., Sensolo, S., and Fazio, F. (2003). A Functional-Anatomical Model for Lipreading. Journal of Neurophysiology 90, 2005-2013.

Perfetti, C. A., and Sandak, R. (2000). Reading optimally builds on spoken language: Implications for deaf readers. Journal of Deaf Studies and Deaf Education 5, 32-50.

Pourtois, G., and de Gelder, B. (2002). Semantic factors influence multisensory pairing: a transcranial magnetic stimulation study. Neuroreport 13, 1567-1573.

Price, C., and McCrory, E. (2005). Functional brain imaging studies of skilled reading and developmental dyslexia. In The science of reading - a handbook, M. Snowling, and C. Hulme, eds. (Oxford, Blackwell Publishing).

Raij, T., Uutela, K., and Hari, R. (2000). Audiovisual integration of letters in the human brain. Neuron 28, 617-625.

Ramus, F. (2003). Developmental dyslexia: specific phonological deficit or general sensorimotor dysfunction? Current Opinion in Neurobiology 13, 212-218.

Rey, A., Ziegler, J. C., and Jacobs, A. M. (2000). Graphemes are perceptual reading units. Cognition $75, \mathrm{~B} 1-12$.

Sams, M., Aulanko, R., Hamalainen, M., Hari, R., Lounasmaa, O. V., Lu, S. T., and Simola, J. (1991). Seeing speech: visual information from lip movements modifies activity in the human auditory cortex. Neuroscience Letters 127, 141-145.

Schulte-Korne, G., Deimel, W., Bartling, J., and Remschmidt, H. (2001). Speech perception deficit in dyslexic adults as measured by mismatch negativity (MMN). International Journal of Psychophysiology 40, 77-87.

Sekiyama, K., Kanno, I., Miura, S., and Sugita, Y. (2003). Auditory-visual speech perception examined by $\mathrm{FMRI}$ and PET. Neuroscience Research 47, 277-287.

Serniclaes, W., Sprenger-Charolles, L., Carre, R., and Demonet, J. F. (2001). Perceptual discrimination of speech sounds in developmental dyslexia. Journal of Speech Language and Hearing Research 44, 384-399.

Serniclaes, W., Van Heghe, S., Mousty, P., Carre, R., and Sprenger-Charolles, L. (2004). Allophonic mode of speech perception in dyslexia. Journal of Experimental Child Psychology 87, 336-361.

Shaywitz, S. E., and Shaywitz, B. A. (2005). Dyslexia (Specific Reading Disability). Biological Psychiatry 57, 1301-1309.

Simos, P. G., Fletcher, J. M., Bergman, E., Breier, J. I., Foorman, B. R., Castillo, E. M., Davis, R. N., Fitzgerald, M., and Papanicolaou, A. C. (2002). Dyslexia-specific brain activation profile becomes normal following successful remedial teaching. Neurology 58, 1203-1213.

Snowling, M. (2000). Dyslexia (Oxford, Blackwell Publishers). 
Snowling, M., and Hulme, C., eds. (2005). The science of reading: a handbook (Oxford, Blackwell Publishing).

Snowling, M. J. (1980). The development of grapheme-phoneme correspondence in normal and dyslexic readers. Journal of Experimental Child Psychology 29, 294-305.

Stein, B. E., and Meredith, M. A. (1993). The merging of the senses (Cambridge, MA, MIT Press).

Stein, B. E., and Wallace, M. T. (1996). Comparisons of cross-modality integration in midbrain and cortex. Progress in Brain Research 112, 289-299.

Sumby, W. H., and Pollack, I. (1954). Visual contribution to speech intelligibility in noise. The Journal of the Acoustical Society of America 26, 212-215.

Torgesen, J. K. (2005). Recent discoveries on remedial interventions for children with dyslexia. In The science of reading - a handbook, M. Snowling, and C. Hulme, eds. (Oxford, Blackwell Publishing).

Treiman, R., and Kessler, B. (2005). Writing systems and spelling development. In The science of reading - a handbook, M. Snowling, and C. Hulme, eds. (Oxford, Blackwell Publishing).

Vellutino, F. R., Fletcher, J. M., Snowling, M. J., and Scanlon, D. M. (2004). Specific reading disability (dyslexia): what have we learned in the past four decades? Journal of Child Psychology and Psychiatry 45, 2-40.

Wallace, M. T., Wilkinson, L. K., and Stein, B. E. (1996). Representation and integration of multiple sensory inputs in primate superior colliculus. Journal of Neurophysiology 76, 1246-1266.

Wright, T. M., Pelphrey, K. A., Allison, T., McKeown, M. J., and McCarthy, G. (2003). Polysensory Interactions along Lateral Temporal Regions Evoked by Audiovisual Speech. Cerebral Cortex 13, 1034-1043. 


\section{Chapter 2}

\section{Integration of letters and speech sounds in the human brain}

This chapter is based on:

Van Atteveldt, N., Formisano, E., Goebel, R., and Blomert, L. (2004). Integration of letters and speech sounds in the human brain. Neuron 43, 271-282. 


\section{Abstract}

Most people acquire literacy skills with remarkable ease even though the human brain is not evolutionarily adapted to this relatively new cultural phenomenon. Associations between letters and speech sounds form the basis of reading in alphabetic scripts. We investigated the functional neuroanatomy of the integration of letters and speech sounds using functional magnetic resonance imaging (fMRI). Letters and speech sounds were presented unimodally and bimodally in congruent or incongruent combinations. Analysis of single-subject data and group data aligned on the basis of individual cortical anatomy revealed that letters and speech sounds are integrated in heteromodal superior temporal cortex. Interestingly, responses to speech sounds in a modality-specific region of the early auditory cortex were modified by simultaneously presented letters. These results suggest that efficient processing of culturally defined associations between letters and speech sounds relies on neural mechanisms similar to those naturally evolved for integrating audiovisual speech. 


\section{Introduction}

Reading is essential to social and economic success in the present technological society (National Reading Council, 1998). In contrast to spoken language, which is a product of biological evolution, reading and writing are cultural inventions from the last few thousand years, and only relevant for most people since a few hundred years (Liberman, 1992). An intriguing question is, therefore, how it is possible that most people acquire literacy skills with such remarkable ease even though a naturally evolved brain mechanism for reading is unlikely to exist. An interesting hypothesis is that evolutionarily adapted brain systems for spoken language provide a neural foundation for reading ability, which is illustrated by the low literacy levels in deaf people (Perfetti and Sandak, 2000).

Nowadays most written languages are speech-based alphabetic scripts, in which speech sound units (phonemes) are represented by visual symbols (letters, or graphemes). Learning the correspondences between letters and speech sounds of a language is therefore a crucial step in reading acquisition, failure of which is thought to account for reading problems in developmental dyslexia (Frith, 1985). However, in the normal situation, letter-speech sound associations are learned and used with high efficiency. At least $90 \%$ of school children learn the letter-sound correspondences without exceptional effort within a few months (Blomert, 2002), which is a remarkable achievement since our brains are not phylogenetically adapted to the requirements for acquiring written language.

Associations between sensory events in different modalities can either be defined by natural relations (e.g., the shape and sound of a natural object), or by more artificial relations. In contrast to the culturally defined associations between letters and speech sounds (Raij et al., 2000), lip reading is based on naturally developed associations of speech with visual information (Paulesu et al., 2003). Therefore, it seems a plausible assumption that the perception of speech and the inherently linked lip movements (hereafter referred to as audiovisual speech) emerged simultaneously during evolution, shaping the brain for integrating this audiovisual information.

At the behavioral level, it has been reported that speech perception can be influenced both by lip movements and by letters. Sumby and Pollack (1954) showed that lip reading can improve speech perception, especially in situations when the auditory input is degraded. More extremely, lip reading can also change the auditory speech percept, as is shown in the McGurk effect (McGurk and MacDonald, 1976). Improvement of speech perception by simultaneous presentation of print has been demonstrated at the level of words (Frost et al., 1988) and syllables (Massaro et al., 1988). Dijkstra and colleagues reported facilitation and inhibition effects on auditorily presented phoneme identity decisions by congruent and incongruent letter primes respectively, suggesting activation of phoneme representations by letters (Dijkstra et al., 1989).

A neural mechanism for the integration of audiovisual speech has been suggested by Calvert and colleagues (Calvert et al., 1999; Calvert et al., 2000) and supported by other neuroimaging findings on audiovisual speech perception (Sams et al., 1991; Sekiyama et al., 2003; Wright et al., 2003) and lip reading (Calvert et al., 1997; Calvert and Campbell, 2003; Paulesu et al., 2003). Results of these studies suggest that the perceptual gain experienced when perceiving multimodal speech is accomplished by 
enhancement of the neural activity in the relevant sensory cortices. The left posterior superior temporal sulcus (STS) has been advanced as the heteromodal site that integrates visual and auditory speech information and modulates the modality-specific cortices by back-projections (Calvert et al., 1999; Calvert et al., 2000). Modalityspecific regions involved in this mechanism are the visual motion processing area V5 and auditory association areas in superior temporal cortex. In addition to this interplay between STS and sensory cortices, frontal and parietal regions seem to be involved, although activation of these regions is less consistent between the different studies. Interestingly, the involvement of the left posterior STS in the integration of auditory and visual nonlinguistic information has also been reported recently (Beauchamp et al., 2004; Calvert et al., 2001). These results suggest that the STS has a more general role in the integration of cross-modal identity information.

As opposed to the integration of lip movements and speech sounds, the neural mechanism that mediates the integration of letters and speech sounds is less clear. As yet, the only neuroimaging study that directly investigated this issue using multimodal stimulation was conducted by Raij and colleagues, who measured magnetic brain responses to unimodally and bimodally presented letters and speech sounds using magnetoencephalography (MEG) (Raij et al., 2000). They report a sequence of processes by which letters and speech sounds may be integrated, starting with modality-specific activation (60-120 ms after stimulus onset) in corresponding sensory cortices, followed by convergence of auditory and visual activations (around $225 \mathrm{~ms}$ ) in lateral midtemporal cortex. Interaction of auditory and visual responses in the right temporo-occipital-parietal junction started at $280 \mathrm{~ms}$, differential interaction effects for matching and nonmatching letters and speech sounds were observed in the STS at 380$540 \mathrm{~ms}$. Although the time course of letter-speech sound integration provided by this study is highly informative, the spatial resolution of MEG limits the accuracy by which the exact locations of brain areas responsible for the different processes can be determined.

In the present study, we use functional magnetic resonance imaging (fMRI) at 3 Tesla to investigate the functional neuroanatomy of the integration of letters and speech sounds with high spatial resolution. Subjects passively listened to and/or viewed unimodally or bimodally presented speech sounds and letters; bimodal stimuli were either congruent or incongruent. The use of a passive perception task has been shown to be efficient by other cross-modal fMRI studies (Calvert et al., 2000; Wright et al., 2003).

The unimodal conditions were used to find brain areas responding to either letters or speech sounds (modality-specific regions), and areas responding to both (convergence regions). The bimodal conditions were used to identify regions responding more to bimodal than to unimodal stimuli (integration), and regions that differently respond to congruent and incongruent combinations of letters and speech sounds (congruency effects). We analyzed our data with different approaches. First, we searched for regions that responded stronger to bimodal ( $A V$, audiovisual) stimulation than to each unimodal condition (V, visual; $\mathrm{A}$, auditory) $[(\mathrm{AV}>\mathrm{A}) \cap(\mathrm{AV}>\mathrm{V})]$ (Beauchamp et al, 2004; Calvert et al., 1999; Hadjikhani and Roland, 1998). Second, we followed the assumption that integration is performed on converging inputs from each modality by multisensory neurons (Meredith, 2002; Raij et al., 2000), by including the constraint 
that integration sites should also respond significantly to both unimodal conditions in isolation [(AV > A $) \cap(A V>V) \cap A \cap V]$. Third, we determined interaction effects $[A V \neq(A+V)]$ in individually defined regions of interest. Finally, we explored the effects of the relatedness of letters and speech sounds by contrasting the congruent and incongruent bimodal conditions.

Functional MRI results are typically presented as group activation maps whereby the individual data are transformed into a standard space and averaged. The high intersubject variability in brain anatomy limits the spatial accuracy of group maps produced in this way. We avoided this limitation in two ways. First, our experimental design and visualization methods allowed us to analyze and present data from individual subjects. Second, we analyzed the group data aligned on the basis of individual cortical anatomy (see Experimental Procedures). A major advantage of cortex-based intersubject alignment above more traditional methods of spatial normalization is that it increases statistical power while preserving high anatomical accuracy in the group results.

Another important aspect of the present study is that the stimuli were presented in silent intervals between subsequent volume scans (see Experimental Procedures). This method minimizes the effects of the acoustic noise produced by fast gradient switching during functional imaging on experimentally evoked auditory activation (Jäncke et al., 2002; Shah et al., 2000). This allows a highly accurate investigation of the contribution of auditory brain regions to cross-modal integration processes and more particularly the processing of letter-speech sound associations.

\section{Results}

\section{Group results - Unimodal conditions}

Figure 2.1A shows the result of the multi-subject multiple linear regression analysis after cortex-based alignment of anatomical and functional data (see Experimental procedures). The statistical map shows the activation for the unimodal predictors versus baseline $[t=8.2, \mathrm{q}(\mathrm{FDR})<0.000]$, color-coded for relative contribution of unimodal visual (green), unimodal auditory (red), and similar contribution of the visual and auditory predictors (yellow) to explaining the signal variance. Unimodal presentation of letters activated the lateral and inferior occipital-temporal cortex; unimodal presentation of speech sounds activated Heschl's gyrus (primary auditory cortex), and regions of the superior temporal gyrus (STG) and the STS. More posteriorly located regions of the STS and STG were activated by both unimodal conditions.

Figure 2.1B shows averaged time courses of the BOLD response corresponding to the regions shown in the relative contribution map (Figure 2.1A). Time courses are shown for three regions with different response patterns: a region in the auditory cortex (Heschl's gyrus) showing modality-specific auditory activation [unimodal auditory > unimodal visual, $\mathrm{q}(\mathrm{FDR})<0.001$ ], a region in posterior STS/STG showing significant response to both unimodal conditions, and a region in the visual cortex (fusiform gyrus) showing modality-specific visual activation [unimodal visual $>$ unimodal auditory, $\mathrm{q}(\mathrm{FDR})<0.001]$. The time courses confirm that the responses in auditory and 
visual cortices were highly modality specific, while the posterior STS/STG showed a heteromodal response pattern. These heteromodal regions in STS and STG are candidate regions for multisensory convergence.

\section{Group results - Bimodal conditions}

The first step in identifying integration sites was to find regions that responded stronger to bimodal stimulation than to each unimodal condition, by performing the conjunction analysis of (bimodal congruent $>$ unimodal auditory) $\cap$ (bimodal congruent $>$ unimodal visual). This conjunction analysis will be referred to as $C 2$. The second step was to constrain this analysis by including the requirement that integration sites should also respond significantly to each modality in isolation. We identified such regions with a conjunction analysis of the following contrasts: (bimodal congruent $>$ unimodal auditory) $\cap$ (bimodal congruent $>$ unimodal visual) $\cap$ (unimodal auditory $>$ baseline) $\cap$ (unimodal visual $>$ baseline). This conjunction analysis will be referred to as $C 4$. To explore the effects of the relatedness of letters and speech sounds, we examined the contrast of bimodal congruent versus bimodal incongruent. This contrast analysis will be referred to as congruency contrast.

Figure 2.2 shows the results of the multisubject random-effect analyses of the congruency contrast $[\mathrm{t}(15)=3.75, \mathrm{q}(\mathrm{FDR})<0.05]$ and the $\mathrm{C} 2$ and $\mathrm{C} 4$ analyses (shown at the same $t$ value). As is shown in Figure $2.2 \mathrm{~A}$ and $2.2 \mathrm{C}$ (green activation maps), the $\mathrm{C} 4$ analysis identified three superior temporal brain areas in the left hemispheres and one in the right hemisphere. The coordinates of corresponding regions in Talairach space are listed in Table 2.1. The averaged BOLD response time course (Figure 2.2B, STS/ STG) indicates that the response to bimodal stimulation was stronger than to auditory and visual stimulation, and the response to both unimodal conditions stronger than baseline. It should be noted that the activation maps of the $\mathrm{C} 2$ and $\mathrm{C} 4$ analyses completely overlap (green regions), except for three regions in the anterior temporal cortex (shown in yellow): the planum polare (PP) bilaterally (left $[-40,-3,-2]$ and right $[47,4,0])$ and left anterior STS $(-49,-11,-7))$ are significant only in the C2 analysis. As is shown in the time course of the BOLD response (fig. 2.2B, PP/ aSTS), only the response to congruent bimodal stimulation is significant in these anterior temporal areas.

The congruency contrast also revealed superior temporal regions in both hemispheres (Figure 2.2A and 2.2D). However, these regions were located more superiorly and anteriorly on the STG, on Heschl's sulcus (HS) and posterior from Heschl's sulcus on the planum temporale (PT) (Duvernoy, 1999; Kim et al., 2000). The BOLD response time courses of these regions (Figure 2.2B, PT/ HS) reveal a highly interesting response pattern. The response to congruent letter-speech sound pairs was stronger than the response to speech sounds alone (ROI-based analyses: $t=2.9, p<0.005$ [right], $t=2.6, p<0.01$ [left]), while the response to incongruent letter-speech sound pairs was reduced relative to the response to speech sounds alone (ROI-based analyses: $\mathrm{t}=5.1, \mathrm{p}<0.000$ [right], $\mathrm{t}=5.4, \mathrm{p}<0.000$ [left]). Furthermore, there is only a very weak response to unimodal visual letters. These observations indicate that the response to speech sounds in early auditory areas (PT/ HS) was modulated by the simultaneous visual presentation of letters, while letters presented in isolation did not activate these areas. No cross-modal modulation effects were observed in the visual cortex. 
Table 2.1.

Talairach Coordinates of Superior Temporal Integration sites, Revealed by the

Conjunction Analyses, and Comparable Regions Reported by Other Studies

\begin{tabular}{lllllc}
\hline Situdy & Stimuli & Region & $x$ & $y$ & $z$ \\
\hline Present study & Letters and speech & Left STS & -54 & -48 & 9 \\
& sounds & Left STS & -46 & -19 & 2 \\
& & Left STG & -43 & -43 & 23 \\
& & Right STG & 52 & -33 & 18 \\
& Letters and speech & Left STS & -53 & -31 & 0 \\
Raij et al. (2000) & Sounds & Right STS & 48 & -31 & 6 \\
Calvert et al. (2000) & Audiovisual speech & Left STS & -49 & -50 & 9 \\
Sekiyama et al. (2003) & Audiovisual speech & Left STS & -56 & -49 & 9 \\
Beauchamp et al. & Complex objects & Left STS/ MTG & -50 & -55 & 7 \\
(2004) & & & -51 & -36 & 9 \\
Calvert et al. (2001) & White noise/ & Left STS & -51 & & \\
\hline
\end{tabular}

\section{Individual results - Interaction effects}

We selected four regions of interest (ROIs) in each subject (if present): left $(n=15)$ and right $(n=12)$ PT/HS (based on the congruency contrast) and left $(n=11)$ and right $(n=$ 9) STS/STG (based on the C4 analysis). Talairach coordinates and significance levels for all selected ROIs in individual subjects are listed in Table 2.2. We estimated b values for all four predictors by individual ROI-based GLM analyses $\left(b_{a}\right.$, unimodal auditory; $b_{v}$, unimodal visual; $b_{a v i}$, bimodal incongruent; $b_{a v c}$, bimodal congruent). Figure 2.3 shows the normalized $b$ values $\left(b_{a}+b_{v}=1\right)$ averaged over subjects for all four predictors in the four ROIs.

Superadditivity $\left[b_{a v}>\left(b_{a}+b_{v}\right)\right]$ was observed for the congruent bimodal condition in the PT/HS bilaterally [left: $\mathrm{t}(14)=2.5, \mathrm{p}=0,025$; right, $\mathrm{t}(11)=2.9, \mathrm{p}=0.014$ ], but not in STS/STG, where $b_{a v c}$ is significantly less than $\left(b_{a}+b_{v}\right)$ in the right hemisphere [left: $\mathrm{t}(10)=-1.7, \mathrm{p}=0,13$; right, $\mathrm{t}(8)=-3.3, \mathrm{p}=0.011]$. However, in the STS/STG, $\mathrm{b}_{\text {avc }}$ was significantly higher than $b_{a}$ [left: $t(10)=10.1, p=0,000$; right $t(8)=9.9, p=0.000$ ] and $b_{v}$ [left: $t(10)=14.7, p=0,000$; right: $\left.\left.t(8)=14.2, p=0.000\right)\right]$. Response suppression $\left(b_{a v}<\left[\max \left(b_{a}, b_{v}\right)\right]\right)$, was observed for $b_{a v i}$ in the PT/HS [left: $t(14)=7.1$, $\mathrm{p}=0.000$; right: $\mathrm{t}(11)=9.0, \mathrm{p}=0.000$ ], but not in the STS/ STG [left: $\mathrm{t}(10)=1.3, \mathrm{p}=$ 0.24 ; right: $t(8)=-1.9, p=0.1]$. In the STS/STG, $b_{a v i}$ was subadditive $\left[b_{a v}<\left(b_{a}+b_{v}\right)\right]$ [left: $\mathrm{t}(10)=-12.9, \mathrm{p}=0.000$; right: $\mathrm{t}(8)=-7.4, \mathrm{p}=0.000$ ]. Figure 2.3 furthermore reveals a difference in relative proportion of the $b$ values of both unimodal conditions in PT/HS and STS/STG. In STS/STG, $b$ values of both unimodal conditions were equally high, while in PT/HS, the $b$ value of the unimodal visual condition was very low relative to the $b$ value of the unimodal auditory condition. It should be noted that the lack of superadditivity in the STS/STG might be due to the strong response to the isolated unimodal conditions (see Discussion). 


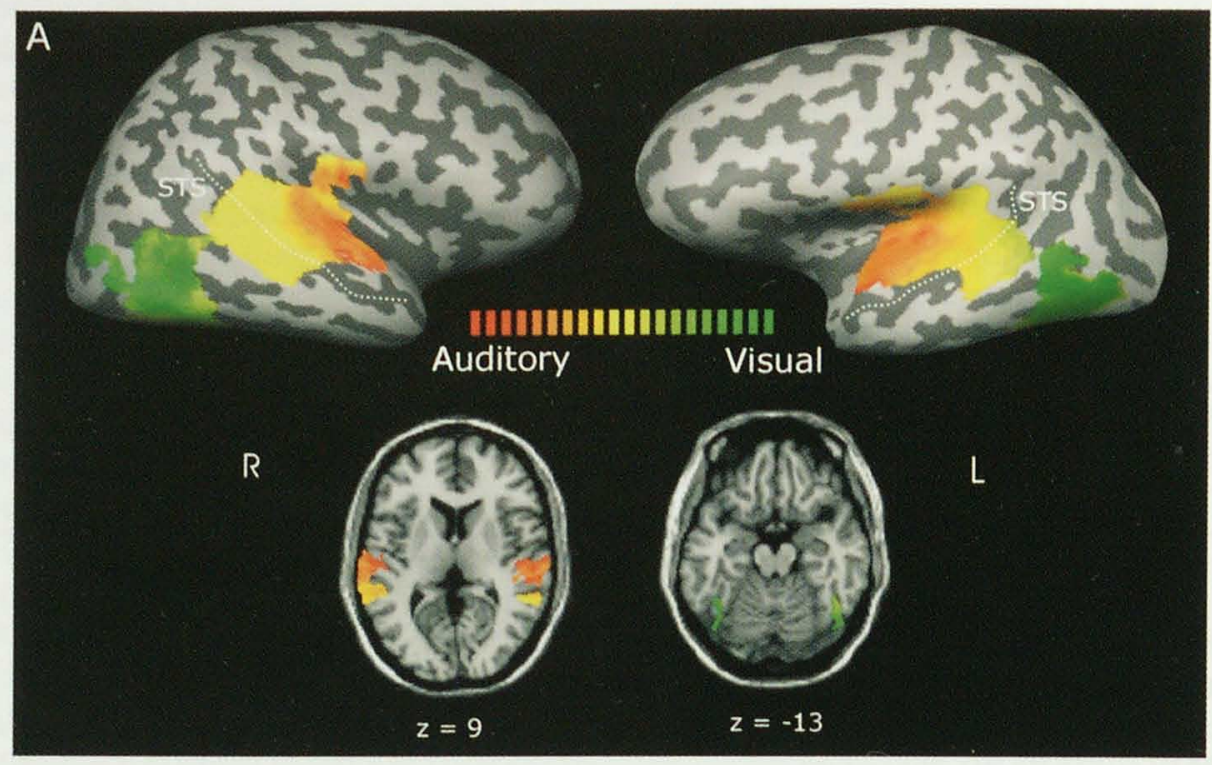

B auditory cortex

Visual cortex

STS/STG
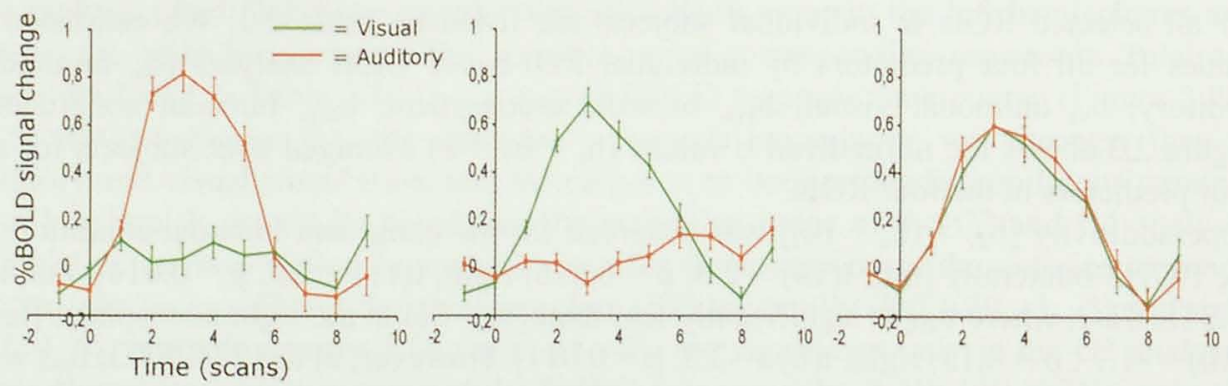

Figure 2.1. Group results for the unimodal conditions

(A) Multi-subject $(n=16)$ general linear model map of the unimodal predictors against baseline performed on the cortex-based aligned functional data. The statistical map is superimposed on the inflated cortical sheet of the individual brain used as target brain for the cortex-based intersubject alignment, and on two axial slices of a template brain normalized in Talairach space. Color coding of the significantly activated voxels indicates relative contribution of auditory (red) and visual (green) unimodal stimulation to the signal change in each voxel. Similar contribution of both unimodal predictors is indicated by yellow.

(B) Averaged time courses of the BOLD response (in percent signal change) during unimodal auditory stimulation (red lines) and unimodal visual stimulation (green lines) in representative regions in auditory cortex, visual cortex and superior temporal sulcus (STS). Error bars indicate SEM. 

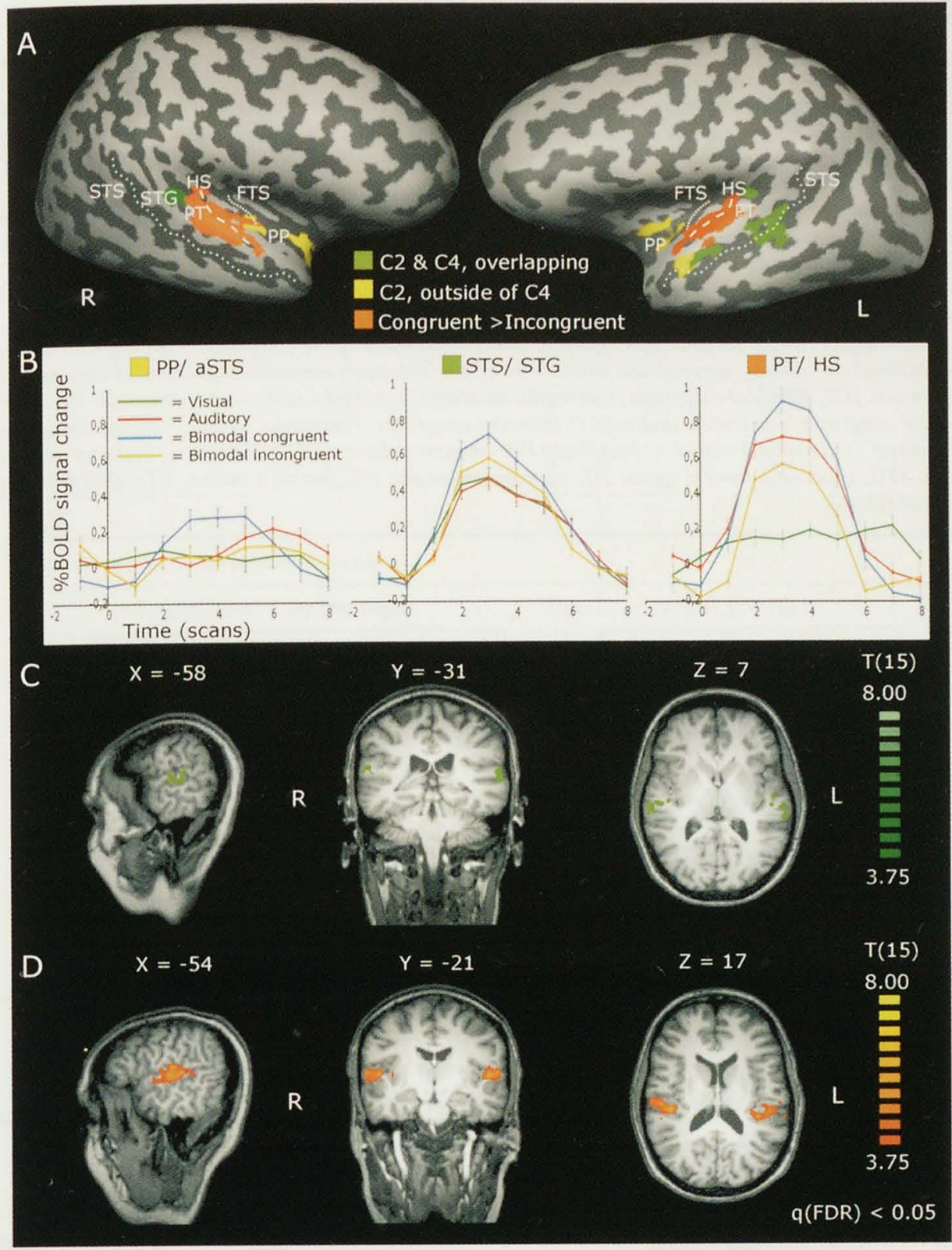

Figure 2.2. Group results for the conjunction and congruency analyses. 
Figure 2.2 (Previous page). Group results for the conjunction and congruency analyses.

(A) Multi-subject $(n=16)$ general linear model maps of the two conjunction analyses (shown in yellow and green), and congruency contrast (shown in orange) performed on the cortex-based aligned functional data. The $C 2$ and $C 4$ maps completely overlap in the green regions; yellow regions are additionally activated in the $C 2$ analysis. Maps are superimposed on the inflated cortical sheet of the individual brain used as target brain for the cortex-based intersubject alignment. The corresponding Talairach coordinates are listed in Table 2.1.

(B) Averaged time courses of the BOLD response (in percent signal change) during bimodal (congruent, blue lines; incongruent, yellow lines) and unimodal conditions (auditory, red lines; visual, green lines) in regions representative for the different maps shown in $(A)$.

(C) Multi-subject $(n=16)$ random-effect general linear model map of the C4 analysis superimposed on sagittal, coronal and axial views of the Talairach normalized anatomical images of the target brain.

(D) Multi-subject ( $n=16$ ) random-effect general linear model map of the congruent $>$ incongruent analysis superimposed on sagittal, coronal and axial views of the Talairach normalized anatomical images of the target brain. [C2, (bimodal congruent $>$ unimodal auditory) $\cap$ (bimodal congruent $>$ unimodal visual); C4, (bimodal congruent $>$ unimodal auditory) $\cap$ (bimodal congruent $>$ unimodal visual) $\cap$ (unimodal auditory $>$ baseline) $\cap$ (unimodal visual $>$ baseline); PP, planum polare; (a)STS, (anterior) superior temporal sulcus; STG, superior temporal gyrus; PT, planum temporale; HS, Heschl's sulcus; FTS, first transverse temporal sulcus].
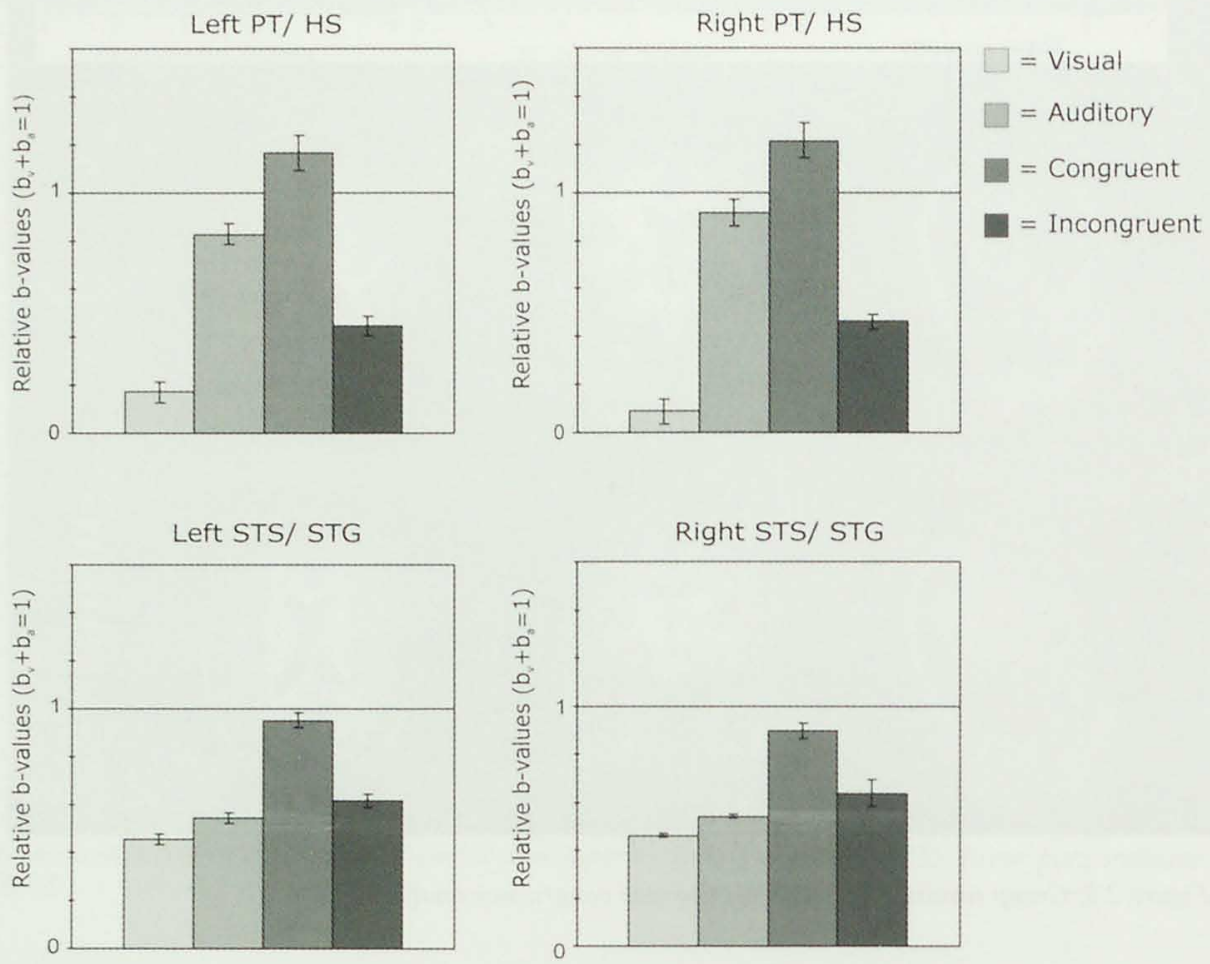

Figure 2.3. Results of the interaction analysis performed on b-values estimated by individual ROI-based GLM analyses. Normalized b-values for all four predictors $\left(b_{y}+b_{a}=1\right)$ are shown for the ROIs in the planum temporale (PT)/Heschl's sulcus (HS) and superior temporal sulcus (STS)/superior temporal gyrus (STG). Lefi PT/HS, $n=15$; right PT/HS, $n=12$; lefi STS/STG, $n=11$; Right STS/STG, $n=9$. Error bars indicate SEM across subjects. 
Table 2.2. Talairach Coordinates and Significance Levels for ROIs in Individual Subjects

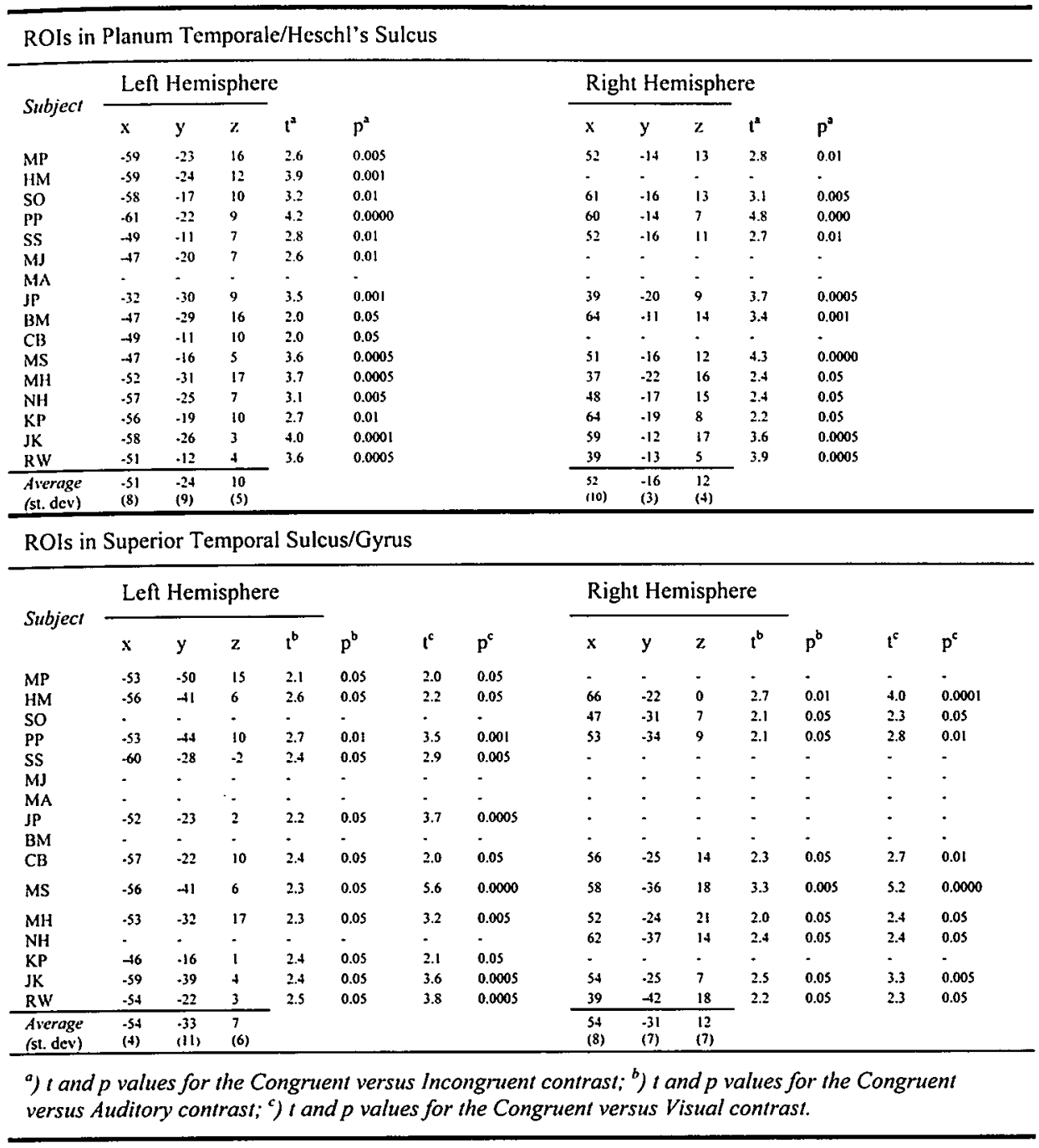

\section{Individual results - detailed localization of modulation effect}

In 15 of the 16 subjects, a region was observed in the left hemisphere PT/HS that showed modulation of the response to speech sounds depending on the congruency of the letters and speech sounds, but no response to unimodally presented letters. Twelve of these 15 subjects showed a similar region in the right hemisphere (see Table 2.2). Figure 2.4 shows the location of the ROIs in PT and HS in six representative individual subjects (dark blue regions), together with the whole region activated by speech sounds (light blue regions). An interesting observation is that only a subregion of the area activated by speech sounds was modulated by visual letter information. In both 
hemispheres, the location of regions showing this modulation effect was highly consistent across all subjects, on HS extending on the PT. This location is just posterior and lateral to the primary auditory cortex which is located on $\mathrm{HG}$, between the first transverse sulcus (FTS) and Heschl's sulcus (Formisano et al., 2003), and may correspond to what has been described as lateral belt cortex in the macaque monkey (Kaas and Hackett, 2000).

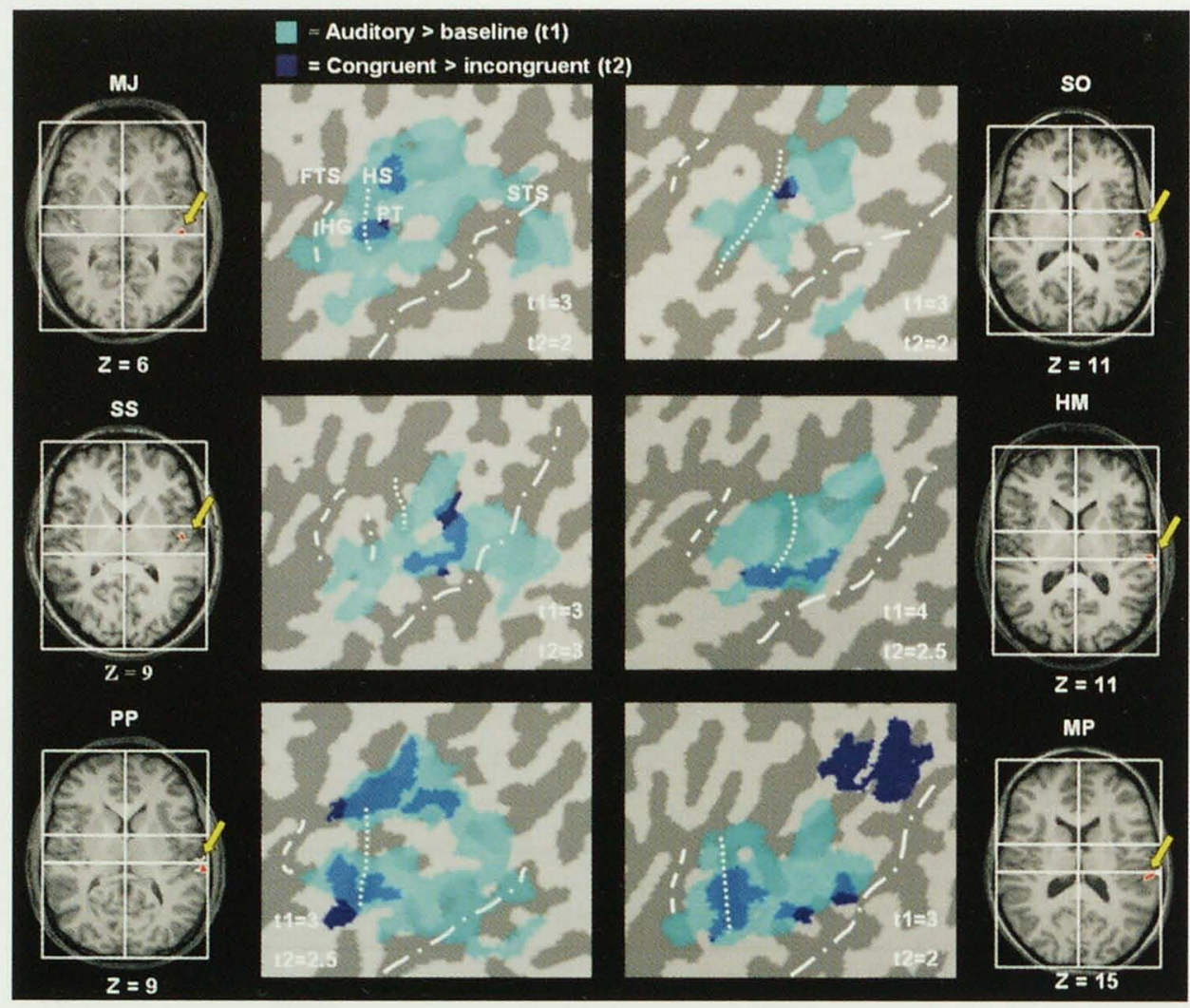

Figure 2.4. Location of ROIs in early auditory cortex in the left hemisphere in six representative individual subjects. Statistical maps for unimodal auditory $>$ baseline (light blue) and bimodal congruent $>$ bimodal incongruent (dark blue) are superimposed on the inflated cortical sheets of the individual subjects. The congruency contrast is also superimposed on axial views of the individual Talairach normalized anatomical images (orange clusters indicated by yellow arrows). $T$ values for both contrasts are given in the figure. STS, superior temporal sulcus; PT, plamum temporale; HS, Heschl's sulcus; FTS, first transverse temporal sulcus. 


\section{Discussion}

The aim of the present study was to investigate the neural correlates of the integration of letters and speech sounds. The most interesting finding is a cross-modal modulation of the response to speech sounds in modality-specific early auditory cortex (HS and PT) by simultaneous visual presentation of congruent or incongruent letters. Integration sites were identified in the STG and STS. These regions showed a heteromodal response pattern, i.e. responded significantly to both unimodal conditions and in addition responded stronger to bimodal than to unimodal letters and speech sounds. Furthermore, anterior temporal regions were revealed that only responded to bimodal congruent letters. A schematic summary of our findings regarding the integration of letters and speech sounds is depicted in Figure 2.5.
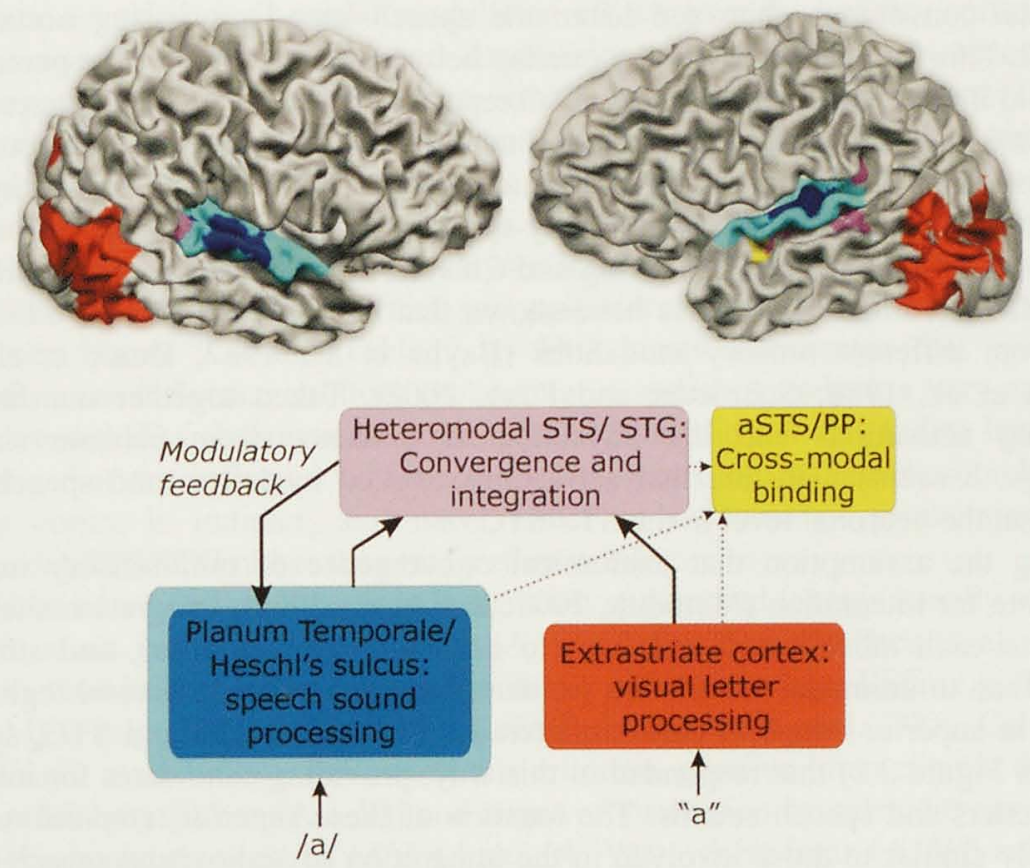

Figure 2.5. Schematic summary of our findings on the integration of letters and speech sounds.

Multi-subject statistical maps are superimposed on the folded cortical sheet of the individual brain used as target brain for the cortex-based intersubject alignment: unimodal visual > unimodal auditory (red), unimodal auditory > unimodal visual (light blue), congruent > incongruent (dark blue), and the conjunction of bimodal $>$ unimodal \& unimodal $>$ baseline (C4, violet). A schematic description of our findings and interpretation based on the functional neuro-anatomical data is shown in the lower panel. Dashed lines indicate that aSTS/PP may either receive input directly from the modality-specific regions, or from the heteromodal STS/STG. (a)STS, (anterior) superior temporal sulcus; STG, superior temporal gyrus; PP, planum polare; /a, speech sound $a$; “ $a$ ", letter $a$. 
Modality-specific activation to letters and speech sounds was found in corresponding sensory processing areas. The inferior occipital-temporal cortex (green regions, Figure 2.1) responded bilaterally to unimodally presented letters but not to unimodally presented speech sounds. This location is consistent with other fMRI studies that investigated single letter processing (Gauthier et al., 2000; Gros et al., 2001; Longcamp et al., 2003; Polk et al., 2002). Modality-specific auditory activation was found in superior temporal cortex (Heschl's gyrus, planum temporale and the middle part of the STG, see red regions in Figure 2.1). These regions were activated by speech sounds but not by letters. The location of these regions is consistent with areas involved in speech sound processing reported by other imaging studies (Demonet et al., 1992; Jäncke et al., 2002; Suzuki et al., 2002; Tervaniemi et al., 2000).

Bilateral regions in the STS and posterior STG responded to the unimodal presentation of both letters and speech sounds. Interestingly, these regions are anatomically located between the visual and auditory processing regions were modality-specific responses were observed (yellow regions, Figure 2.1). These observations indicate that multimodal convergence between letter and speech sound processing occurs in the STS/STG. This is consistent with the overlap between activations for the perception of speech and lip movements in STS and STG reported by Wright and colleagues (Wright et al., 2003). Because large numbers of neurons are sampled simultaneously with fMRI, responsiveness of a region to stimulation in different modalities may reflect responses of subpopulations of modality-specific neurons within the same voxels instead of neuronal convergence (King and Calvert, 2001; Meredith, 2002). However, single-cell recordings in primates have shown that neurons in STS and STG receive inputs from different sensory modalities (Baylis et al., 1987; Bruce et al., 1981; Hikosaka et al., 1988; Schroeder and Foxe, 2002). Taken together our finding of converging activations and the physiological evidence from primate single-cell recordings, it seems plausible that activations evoked by letters and speech sounds converge at the neuronal level in the STS/STG.

Following the assumption that anatomical convergence of multisensory input is a prerequisite for integration (Meredith, 2002; Raij et al., 2000), integration sites should respond to each modality in isolation (to determine convergence), and stronger to bimodal than to unimodal stimulation (to determine integration). Several regions were revealed in superior temporal cortex bilaterally (located on STS and STG, see green regions in Figure 2.2) that responded in this way, providing candidates for integration sites of letters and speech sounds. The location of these superior temporal regions is remarkably similar to those involved in the integration of audiovisual speech (Calvert et al., 2000; Sekiyama et al., 2003; Wright et al., 2003), audiovisual information about complex objects (Beauchamp et al., 2004) and temporally matched meaningless audiovisual stimuli (Calvert et al., 2001) (see Table 2.1). Our results therefore support the general role of the STS/STG in integrating audiovisual identity information (Beauchamp et al., 2004; Calvert, 2001).

Our analyses also revealed areas which were active during bimodal stimulation but neither during visual nor auditory unimodal stimulation. Brain areas with such a response profile would indicate to contain cells exhibiting conjunctive coding (Hinton et al., 1986). Without the constraint that modality-specific responses should be significant, we indeed revealed additional activation clusters, located in the anterior 
temporal cortex (see Figure 2.2A, yellow regions). Interestingly, these regions responded only during congruent but not during incongruent bimodal stimulation (see Figure 2.2B). This unexpected selectivity for congruent bimodal stimulation might indicate that these regions signal the successful binding of cross-modal information into a unified percept. Future work is necessary to investigate the detailed properties of these regions. It would, for example, be interesting to know whether some or all subregions are specifically involved in coding congruent letter-sound stimulation or whether they would also respond during other congruent, but not incongruent, visual and auditory information. Furthermore, it would be interesting to find out whether these anterior temporal regions receive input directly from the modality-specific processing regions, which is expected if conjunctive coding indeed takes place, or whether the selective response to congruent information is determined by input from heteromodal STS/STG.

To explore the effects of relatedness between the stimuli in both modalities, we examined the contrast of congruent versus incongruent bimodal stimulation. This comparison revealed cross-modal modulation effects in early auditory cortex (HS and PT), expressed by enhancement and suppression of the response to speech sounds depending on the congruency of the simultaneously presented letters. Congruent combinations of letters and speech sounds elicited a response that significantly exceeded the response to speech sounds alone, while the response to incongruent combinations of letters and speech sounds was significantly weaker than the response to speech sounds alone. Individual analyses indicated a highly consistent localization of this cross-modal modulation effect, just posterior and lateral to the primary auditory cortex (see Figure 2.4 and Table 2.2). This location indicates that visual letter information can influence auditory processing of speech sounds at a very early stage. Another remarkable observation is that these regions did not respond to letters alone, suggesting that the influence of letters on the processing of speech sounds in early auditory cortex is indirect, and may reflect an outcome of prior integration in heteromodal STS/STG rather than integration itself.

It should be noted that multisensory integration at neuronal level is usually inferred on the basis of an interaction test $[A V \neq(A+V)]$ (see e.g., Wallace et al., 1996). A similar method has recently been used to detect integration from EEG (e.g., Fort et al., 2002), MEG (e.g., Raij et al., 2000) and fMRI data (Calvert et al., 2000; Calvert et al., 2001; Wright et al., 2003). Following this criterion, positive interaction is determined by superadditivity $[\mathrm{AV}>(\mathrm{A}+\mathrm{V})]$ and negative interaction by subadditivity $[\mathrm{AV}<(\mathrm{A}$ $+\mathrm{V})]$ or response suppression $(\mathrm{AV}<[\max (\mathrm{A}, \mathrm{V})])$, where $[\max (\mathrm{A}, \mathrm{V})]$ indicates the largest of the unimodal responses (Calvert et al., 2001). We tested for interaction effects using the $b$. values for the four predictors estimated by single-subject GLM analyses in selected ROIs in STS/STG and PT/HS (Figure 2.3). Our results show that in left and right PT/HS, the $b$ value of the congruent bimodal predictor is superadditive and the $b$ value of the incongruent bimodal predictor shows response suppression. In the left and right STS/STG, the $b$ value of the congruent bimodal predictor is significantly higher than either unimodal $b$ value, but does not reach superadditivity. Furthermore, the $b$ value of the incongruent bimodal predictor is subadditive, but does not show response suppression. 
However, results from interaction analyses performed on fMRI data should be interpreted with caution (Beauchamp et al., 2004; Calvert, 2001; King and Calvert, 2001; Wright et al., 2003). Because of the intrinsic nature of the BOLD response and its limited dynamic range, it is possible that a superadditive change in the response at the neuronal level is not reflected in a similarly superadditive change in the BOLD response. Therefore, superadditivity is more likely to be found when the response to one or both of the unimodal conditions is weak or absent (or even negative), than when the BOLD response to both modalities is separately already strong. This prediction is confirmed by the findings of Wright et al. (2003), and by the different response patterns we find in the anterior temporal cortex, PT/HS, and the STS/STG (see Figure 2.2B and Figure 2.3). In the PT/HS, the very weak response to unimodal visual stimuli leaves opportunity for congruent bimodal stimuli to evoke a super-additive BOLD response. In the anterior temporal cortex, neither unimodal condition evokes a significant response, so the response evoked by the congruent bimodal condition exceeds the sum of the unimodal responses even more extremely. Conversely, the response to both unimodal conditions is considerable in the STS/STG, preventing the increased response to bimodal congruent stimulation from reaching superadditivity. These observations clearly indicate the importance of also inspecting the unimodal responses when interpreting interaction effects. Because of the difficulties in interpreting interactions measured with $\mathrm{fMRI}$ we decided to use the conjunction analyses to determine cross-modal integration at a map level. A similar approach for inferring cross-modal integration from fMRI data has been used by another recent fMRI study (Beauchamp et al., 2004). Based on the combination of our conjunction and congruency analyses we suggest that integration of letters and speech sounds is performed in heteromodal regions in STS/STG and that the outcome of this integration process is projected back to the putative "modality-specific" auditory regions in HS and PT, and modulates the processing of speech sounds.

The idea of interplay between modality-specific and heteromodal brain areas during cross-modal integration, is supported by other fMRI findings (Bushara et al., 2003; Macaluso et al., 2000; Calvert et al., 1999; Calvert et al., 2000). The cross-modal modulation effect we observe in phoneme-processing regions in the auditory cortex is consistent with the suggestion that integration sites in STS/STG modulate processing in sensory cortices by feedback projections during audiovisual speech perception (Calvert et al., 1999; Calvert et al., 2000). We think that the enhancement (suppression) of activity observed in these regions in the case of congruent (incongruent) visual presentation of letters is a consequence of the presence of language-related audiovisual functional connections developed during reading acquisition and daily used. Whether and where in the brain similar effects are present for other audio-visual pairs is an interesting empirical question that deserves to be investigated in future studies. In sum, our findings indicate that the integration of letters and speech sounds is performed by a neural mechanism similar to the mechanism for the integration of speech with lip movements (or possibly an even more general audiovisual integration system). This similarity is crucial and profound, since it supports the hypothesis that the efficient use of letter-sound associations is possible because the brain can make use of a naturally evolved mechanism for associating visual information with speech. 
A feedback mechanism implies that the modulation effect observed in HS and PT should occur later in time than the convergence and integration in STS/STG. Unfortunately, fMRI does not provide temporal information that is accurate enough to verify this implication. However, the time-course information on audiovisual letter integration provided by Raij and colleagues (Raij et al., 2000) does support a feedback mechanism: convergence and interaction effects started at $225 \mathrm{~ms}$, while differences in interaction effects for matching and nonmatching letters and speech sounds did not occur until 380-450 ms. More direct support for our proposed feedback mechanism comes from a recent report by Schroeder and Foxe (2002). They investigated the laminar profile and multisensory response properties of neurons in the posterior auditory association cortex (presumably corresponding to our PT/HS regions) and the superior temporal sulcus (presumably corresponding to our STS/STG regions) of the macaque monkey. In auditory association cortex, a laminar input profile for visual stimulation was observed indicating feedback projections. In the STS, laminar profiles indicated feedforward convergence of visual and auditory information. Consistent information was provided by the response latencies of visual and auditory input: Responses to visual and auditory stimulation in the STS were coincident, while visual responses were delayed relative to auditory responses in the auditory association cortex.

The modulation effect observed in the auditory association cortex was not observed in the visual association cortex. This implies that the outcome of the integration of letters and speech sounds is projected back to influence selectively only early auditory processing levels, but not lower-level visual processing. In contrast, both sensory systems seem to be related reciprocally during audiovisual speech perception (Calvert et al., 1999). An asymmetry in the representations of associations between letters and speech sounds has been reported at the behavioral level before (Dijkstra et al., 1993; Hardy et al., 1972). A possible explanation for the observed unidirectional influence is that speech sounds are continuous in time and more variable, and therefore more difficult to recognize than discrete and invariable letter symbols (Liberman, 1992). This is consistent with the finding of Sekiyama et al. (2003) that visual information exerts a stronger influence on auditory processing when speech is less intelligible. The direction of modulatory effects between letters and speech sounds may depend on the temporal synchrony of the stimuli (Dijkstra et al., 1993; Jones and Callan, 2003), a possibility that will need further investigation.

A remark should be made about the possible involvement of frontal and parietal brain regions in the integration of letters and speech sounds. Frontal (Broca's area, premotor cortex, anterior cingulate cortex) and parietal (inferior and posterior parietal cortex) activation has been reported during lip reading and audiovisual speech perception (Calvert et al., 1997; Calvert and Campbell, 2003; Calvert et al., 2000; Jones and Callan, 2003; Olson et al., 2002; Paulesu et al., 2003; Sekiyama et al., 2003), and are associated with speech sound processing and attention. Because of methodological considerations (see Experimental procedures), our functional scans did not cover the whole brain. Therefore, we can not exclude the possibility that frontal and parietal brain regions may also play a role in the proposed mechanism for integration of letters and speech sounds. 


\section{Conclusions}

By using an $\mathrm{fMRI}$ design that allowed investigation of processing in auditory, visual and heteromodal temporal brain regions with high spatial accuracy, we revealed a functional neuronal mechanism for the integration of letters and speech sounds. Modality-specific processing was observed in superior temporal and occipital-temporal cortices, convergence and integration in the heteromodal STS/STG. Furthermore, we revealed anterior temporal regions that exclusively responded to bimodal congruent letters. A most interesting finding was that subregions of early auditory cortex involved in speech sound processing, located on Heschl's sulcus and the planum temporale, were influenced by the congruency of simultaneously presented letters and speech sounds. Because these regions did not respond to letters alone, we interpret this influence as feedback modulation from heteromodal STS/STG regions where integration took place. Our data show that the integration of culturally defined associations between letters and speech sounds recruits a neural mechanism similar to the naturally evolved neural mechanism for the integration of speech information with lip movements.

\section{Experimental procedures}

\section{Subjects}

Sixteen healthy native Dutch subjects ( 3 male, mean age $22 \pm 2.4$, range 19-27) participated in the present study. All subjects were university students enrolled in an undergraduate study program. We selected subjects based on a questionnaire including questions concerning (present or history of) reading or other language problems. All were right-handed, had normal or corrected-to-normal vision, and normal hearing capacity. Subjects gave informed written consent and were paid for their participation.

\section{Stimuli and task design}

Stimuli were speech sounds corresponding to single letters, and visually presented single letters. Speech sounds were digitally recorded (sampling rate $44.1 \mathrm{kHz}, 16$ bit quantization) from a female speaker. Recordings were band-pass filtered $(180-10,000$ $\mathrm{Hz}$ ) and resampled at $22.05 \mathrm{kHz}$. Only speech sounds that were recognized $100 \%$ correctly in a pilot experiment $(n=10)$ were selected for the fMRI experiment. Selected consonants were: $b, d, g, h, k, l, n, p, r, s, t, z$. Selected vowels were: a, e, i, y, $o$, u. Average duration of the speech sounds was $352( \pm 5) \mathrm{ms}$, the average sound intensity level was $71.3( \pm 0.2) \mathrm{dB}$. Visual stimuli were lower-case letters corresponding to the 18 speech sounds. White letters were presented for $350 \mathrm{~ms}$ on a black background in the center of a computer screen, printed in font "Arial" at lettersize 40 . During fixation periods and scanning, a white fixation cross was presented in the center of the screen.

Stimuli were presented in blocks of four different conditions: unimodal visual, unimodal auditory, bimodal congruent and bimodal incongruent. In the bimodal conditions, visual letters and speech sounds were presented simultaneously. Stimulus presentation was synchronized with the scanner pulses using the software package "Presentation" (http://neurobehavioralsystems.com). Experimental blocks (20 s) were 
composed of 4 mini-blocks of 5 seconds (see Figure 2.6). In the first 1.1 seconds of each mini-block, one brain volume was acquired (see "scanning procedure"). No stimuli were presented in this period, only a fixation cross to keep the eyes of the subjects focused on the center of the screen. In the subsequent silent 3.9 seconds, 5 stimuli were presented with a stimulus onset asynchrony (SOA) of $700 \mathrm{~ms}$. This stimulus presentation design was adapted from Jäncke et al. (2002).

Vowels and consonants were presented in separate blocks. Every experimental condition was repeated twice in each run. Experimental blocks were alternated with fixation periods of 20 seconds, every run started and ended with a fixation period. Two runs ( 80 trials per condition) were acquired in $7 / 16$ subjects, four runs (160 trials per condition) were acquired in $9 / 16$ subjects. The order of the experimental blocks was pseudo-randomized within runs and counterbalanced across runs.

Subjects passively listened to and/or viewed the stimuli. Since we were interested in fundamental brain responses to the processing and integration of letters and speech sounds, a passive perception design was chosen because it avoids interaction between activity related to stimulus processing and task-related activity due to cognitive factors (Calvert et al., 2000).

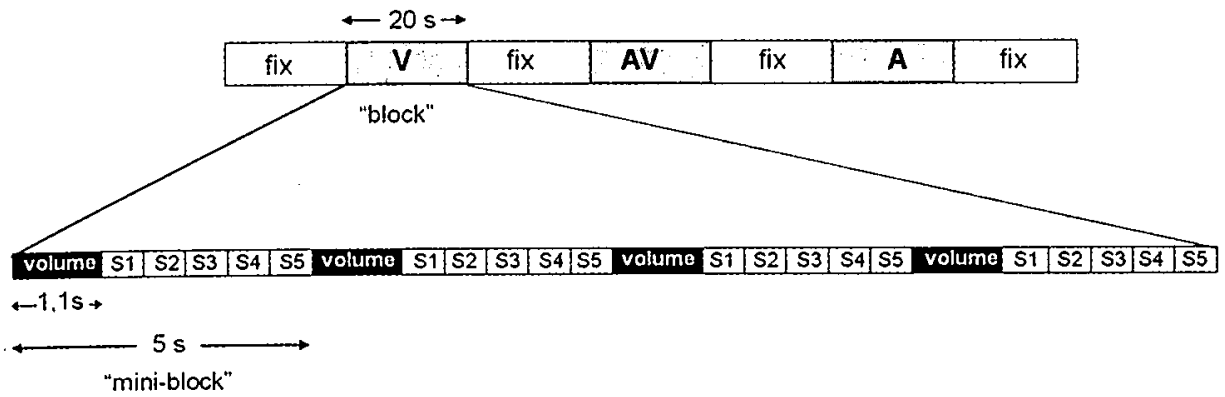

Figure 2.6. Schematic description of the experimental design.

Experimental blocks of $20 \mathrm{~s}$ were composed of 4 mini-blocks of $5 \mathrm{~s}$. Each mini-block starts with the acquisition of one volume scan followed by 5 stimuli presented in silence. Experimental blocks were alternated with fixation periods of $20 \mathrm{~s}$. fix, fixation period; $V$, visual experimental block; $A V$, audiovisual experimental block; $A$, auditory experimental block; $S$, stimulus.

\section{Scanning procedure}

Imaging was performed on a 3 Tesla whole-body system (Magnetom Trio, Siemens Medical Systems, Erlangen, Germany). In each subject, 2 or 4 runs of 71 volumes were acquired using a BOLD-sensitive EPI sequence (matrix: $64 \times 64 \times 18$, voxel size: $3 \times 3 \times 4$ $\mathrm{mm}^{3}$, FOV: $192 \mathrm{~mm}^{2}$, TE/TRslice $=32 / 63 \mathrm{~ms}, \mathrm{FA}=75^{\circ}$ ). Sequence scanning time was $1.1 \mathrm{~s}$, inter-scan gap was $3.9 \mathrm{~s}$, resulting in a TR (sequence repeat time) of $5 \mathrm{~s}$. A slab of 18 axial slices (slab thickness: $7.2 \mathrm{~cm}$ ) was positioned in each individual such that the entire visual and auditory cortices were covered, based on anatomical information from a scout image of 7 sagitally oriented slices. In most of the subjects, the slab did 
not cover the whole brain, excluding the superior frontal and parietal cortices. We reduced the number of slices to maximize scanning speed and silent delay time for stimulus presentation without increasing excessively the volume TR. A high-resolution structural scan (voxel size: $1 \times 1 \times 1 \mathrm{~mm}^{3}$ ) was collected for each subject using a T1weighted 3D MP-RAGE sequence (Magnetization-Prepared Rapid Acquisition Gradient Echo, $\mathrm{TR}=2.3 \mathrm{~s}, \mathrm{TE}=3.93 \mathrm{~ms}$, 192 sagittal slices). Clustering of the volume acquisition in the beginning of each TR provides a period of silence $(3.9 \mathrm{~s}$ in our experiment) between successive scans in which stimulus perception is uncontaminated by EPI noise. This stimulation procedure is demonstrated to be highly efficient for studying the auditory cortex with fMRI (Jäncke et al., 2002; Shah et al., 2000).

\section{Image analysis and visualization}

Functional and anatomical images were analyzed using BrainVoyager 2000 and BrainVoyager QX (Brain Innovation, Maastricht, The Netherlands). The following preprocessing steps were performed: slice scan time correction (using sinc interpolation), linear trend removal, temporal high pass filtering to remove lowfrequency nonlinear drifts of 3 or less cycles per time course, and 3D motion correction to detect and correct for small head movements by spatial alignment of all volumes to the first volume by rigid body transformations. Estimated translation and rotation parameters were inspected and never exceeded $1 \mathrm{~mm}$. Functional slices were coregistered to the anatomical volume using position parameters from the scanner and manual adjustments to obtain optimal fit, and transformed into Talairach space.

For visualization of the statistical maps, all individual brains were segmented at the gray/white matter boundary (using a semi-automatic procedure based on intensity values), and the cortical surfaces were reconstructed, inflated, and flattened. To improve the spatial correspondence mapping between subjects' brains beyond Talairach space matching, the reconstructed cortices were aligned using curvature information reflecting the gyral/sulcal folding pattern. While functional areas do not precisely follow cortical landmarks, it has been shown that a cortical matching approach substantially improves statistical analysis across subjects by reducing anatomical variability (Fischl et al., 1999). Following Fischl et al. (1999), we morphed the reconstructed folded cortical representations of each subject and hemisphere into a spherical representation, which provides a parameterizable surface well-suited for across-subject non-rigid alignment. Each vertex on the sphere (spherical coordinate system) corresponds to a vertex of the folded cortex (Cartesian coordinate system) and vice versa. The curvature information computed in the folded representation is preserved as a curvature map on the spherical representation. The curvature information (folding pattern) is smoothed along the surface to provide spatially extended gradient information driving intercortex alignment minimizing the mean squared differences between the curvature of a source and a target sphere. The alignment proceeds iteratively following a coarse-to-fine matching strategy, which starts with highly smoothed curvature maps and progresses to only slightly smoothed representations. Starting with a coarse alignment as provided by Talairach space, this method ensures that the smoothed curvature of the two cortices possess enough overlap for a locally operating gradient-descent procedure to converge without user intervention. Visual inspection and a measure of the averaged mean squared curvature difference revealed that the alignment of major gyri and sulci was achieved reliably by 
this method. Smaller structures, visible in the slightly smoothed curvature maps, were not completely aligned reflecting idiosyncratic differences between the subjects' brains, such as continuous versus broken characteristics. The established correspondence mapping between vertices of the cortices was used to align the time courses for multi-subject GLM data analysis.

Single-subject and multi-subject analyses were performed by multiple linear regression of the BOLD-response time course in each voxel using four predictors: unimodal visual, unimodal auditory, bimodal congruent and bimodal incongruent. Predictor timecourses were adjusted for the hemodynamic response delay by convolution with a hemodynamic response function (Boynton et al., 1996) (delta 2.5, tau 1.25). Randomeffect analyses were performed on the group data $(n=16)$, enabling generalization of the statistical inferences to the population level. Multi-subject statistical maps were thresholded using the false discovery rate (FDR, (Genovese et al., 2002)). For ROIbased analyses, $t$ statistics and corresponding $p$ values are reported.

To reveal modality-specific responses, the unimodal visual and unimodal auditory predictors were contrasted against baseline. Results of the unimodal predictors against baseline were visualized using a two set relative contribution map (Figure 2.1). The whole colored region in these maps depicts significant explanation of the signal variance by the visual or auditory predictors against baseline, the color coding within this map indicates where the visual predictor contributes more to explaining the variance, where the auditory predictor contributes more, and where they both contribute equally. The relative contribution value in each voxel was computed by $\left(b_{v}-\right.$ $\left.b_{a}\right) /\left(b_{v}+b_{a}\right)$.

To identify integration sites, we used conjunction analyses to extract conjoint activation in the bimodal conditions contrasted to each unimodal condition. At each voxel, a new statistical value was computed as the minimum of the statistical values obtained from all the specified contrasts. The first step in identifying integration sites was to find voxels that were significantly more active during audiovisual stimulation as compared to both modalities in isolation. For this purpose, we performed the conjunction analysis with the following two contrasts: (bimodal congruent $>$ unimodal auditory) $\cap$ (bimodal congruent $>$ unimodal visual). In the second step, we included the constraint that integration sites should show convergence of auditory and visual activity, i.e. respond to both unimodal conditions in isolation. In other words, two contrasts were added to the conjunction analysis: (bimodal congruent $>$ unimodal auditory) $\cap$ (bimodal congruent $>$ unimodal visual) $\cap$ (unimodal auditory $>$ baseline) $\cap$ (unimodal visual $>$ baseline).

To determine interaction effects, we estimated $b$ values for all four predictors by individual ROI-based GLM analyses, and contrasted the bimodal predictors to the sum of the unimodal $b$ values $\left(b_{a}+b_{v}\right)$. For this purpose, we normalized all $b$ values to by dividing them by $\left(b_{a}+b_{v}\right)$. After this normalization, superadditivity is defined by $b_{a v}>$ 1 , and subadditivity by $b_{a v}<1$, and response suppression by $\left(b_{a v}<\left[\max \left(b_{a}, b_{v}\right)\right]\right)$, where $\left[\max \left(b_{a}, b_{v}\right)\right]$ indicates the largest of the unimodal responses (Calvert et al., 2001). We tested for superadditivity and subadditivity by one-sample $t$ tests $\left(b_{a v}\right.$ against $1)$, and for response suppression by paired-samples $t$ tests [ $b_{a v}$ against $\max \left(b_{a}, b_{v}\right]$. 
To find out which brain regions respond differently to congruent and incongruent combinations of letters and speech sounds, the contrast of bimodal congruent versus bimodal incongruent was performed.

\section{Acknowledgements}

The authors are grateful to Holger Mitterer for his help with preparing the stimuli, to Paul Gaalman for his technical assistance and to Peter Hagoort for providing access to the facilities of the F.C Donders Center. The reported research was supported by grant 048.011 .046 of the Dutch Organisation for Scientific Research (NWO). 


\section{References}

Baylis, G. C., Rolls, E. T., and Leonard, C. M. (1987). Functional subdivisions of the temporal lobe neocortex. Journal of Neuroscience 7, 330-342.

Beauchamp, M., Lee, K., Argall, B., and Martin, A. (2004). Integration of auditory and visual information about objects in superior temporal sulcus. Neuron 4l, 809-823.

Blomert, L. (2002). Dyslexie: Stand van Zaken (Dyslexia: State of Affairs in The Netherlands). Report for the Dutch Ministry of Health. In Dyslexie naar een vergoedingsregeling, R. Reij, ed. (Amstelveen, Dutch Health Care Insurance Board).

Boynton, G. M., Engel, S. A., Glover, G. H., and Heeger, D. J. (1996). Linear Systems Analysis of Functional Magnetic Resonance Imaging in Human VI. Journal of Neuroscience 16, 4207 4241.

Bruce, C., Desimone, R., and Gross, C. G. (1981). Visual properties of neurons in a polysensory area in superior temporal sulcus of the macaque. Journal of Neurophysiology 46, 369-384.

Bushara, K. O., Hanakawa, T., Immisch, I., Toma, K., Kansaku, K., and Hallett, M. (2003). Neural correlates of cross-modal binding. Nature Neuroscience 6, 190-195.

Calvert, G. A. (2001). Crossmodal Processing in the Human Brain: Insights from Functional Neuroimaging Studies. Cerebral Cortex 11, 1110-1123.

Calvert, G. A., Brammer, M. J., Bullmore, E. T., Campbell, R., Iversen, S. D., and David, A. S. (1999). Response amplification in sensory-specific cortices during crossmodal binding. Neuroreport 10, 2619-2623.

Calvert, G. A., Bullmore, E. T., Brammer, M. J., Campbell, R., Williams, S. C., McGuire, P. K., Woodruff, P. W., Iversen, S. D., and David, A. S. (1997). Activation of auditory cortex during silent lipreading. Science 276, 593-596.

Calvert, G. A., and Campbell, R. (2003). Reading Speech from Still and Moving Faces: The Neural Substrates of Visible Speech. Journal of Cognitive Neuroscience 15, 57-71.

Calvert, G. A., Campbell, R., and Brammer, M. J. (2000). Evidence from functional magnetic resonance imaging of crossmodal binding in the human heteromodal cortex. Current Biology 10, 649-657.

Calvert, G. A., Hansen, P. C., Iversen, S. D., and Brammer, M. J. (2001). Detection of audio-visual integration sites in humans by application of electrophysiological criteria to the BOLD effect. Neuroimage 14, 427-438.

Demonet, J. F., Chollet, F., Ramsay, S., Cardebat, D., Nespoulous, J. L., R Wise, Rascol, A., and Frackowiak, R. (1992). The anatomy of phonological and semantic processing in normal subjects. Brain $1 / 5,1753-1768$.

Dijkstra, A., Schreuder, R., and Frauenfelder, U. H. (1989). Grapheme context effects on phonemic processing. Language and Speech 32, 89-108.

Dijkstra, T., Frauenfelder, U. H., and Schreuder, R. (1993). Bidirectional grapheme-phoneme activation in a bimodal detection task. Journal of Experimental Psychology: Human Perception and Performance 19, 931-950.

Driver, J., and Spence, C. (2000). Multisensory perception: Beyond modularity and convergence. Current Biology 10, R731-R735.

Duvernoy, H. M. (1999). The Human Brain: surface, three-dimensional sectional anatomy with MRI, and blood supply, Second, completely revised and enlarged edition (Wien New York, Springer-Verlag).

Fischl, B., Sereno, M. I., Tootel, R. B. H., and Dale, A. M. (1999). High-resolution intersubject averaging and a coordinate system for the cortical surface. Human Brain Mapping 8, 272-284.

Formisano, E., Kim, D.-S., Di Salle, F., van de Moortele, P.-F., Ugurbil, K., and Goebel, R. (2003). Mirror-symmetric tonotopic maps in human primary auditory cortex. Neuron 40, 859-869. 
Fort, A., Delpuech, C., Pernier, J., and Giard, M. H. (2002). Dynamics of cortico-subcortical crossmodal operations involved in audio-visual object detection in humans. Cerebral Cortex 12, 1031-1039.

Frith, U. (1985). Beneath the surface of developmental dyslexia. In Surface dyslexia, K. E. Patterson, J. C. Marshall, and M. Coltheart, eds. (London, Routledge \& Kegan-Paul).

Frost, R., Repp, B. H., and Katz, L. (1988). Can speech perception be influenced by simultaneous presentation of print? journal of memory and language 27, 741-755.

Gauthier, I., Tarr, J., Moylan, J., Skudlarski, P., Gore, C., and Anderson, W. (2000). The Fusiform "Face Area" is Part of a Network that Processes Faces at the Individual Level. Journal of Cognitive Neuroscience 12, 495-504.

Genovese, C., Lazar, N., and Nichols, T. (2002). Thresholding of statistical maps in functional neuroimaging using the false discovery rate. Neurolmage 15, 870-878.

Gros, H., Boulanouar, K., Viallard, G., Cassol, E., and Celsis, P. (2001). Event-related functional magnetic resonance imaging study of the extrastriate cortex response to a categorically ambiguous stimulus primed by letters and familiar geometric figures. Journal of Cerebral Blood Flow \& Metabolism 21, 1330-1341.

Hadjikhani, N., and Roland, P. E. (1998). Cross-Modal Transfer of Information between the Tactile and the Visual Representations in the Human Brain: A Positron Emission Tomographic Study. The Journal of Neuroscience $18,1072-1084$.

Hardy, M. H., Smythe, P. C., Stennet, R. G., and Wilson, H. R. (1972). Developmental patterns in elemental reading skills: phoneme-grapheme and grapheme-phoneme correspondences. Journal of Educational Psychology 63, 433-436.

Hikosaka, K., Iwai, E., Saito, H., and Tanaka, K. (1988). Polysensory properties of neurons in the anterior bank of the caudal superior temporal sulcus of the macaque monkey. Journal of Neurophysiology 60, 1615-1637.

Hinton, G. E., McClelland, J. L., and Rumelhart, D. E. (1986). Distributed representations. In Parallel distributed processing: Explorations in the microstructure of cognition, D. E. Rumelhart, and J. L. McClelland, eds. (Cambridge, Massachusetts, The MIT Press).

Jäncke, L., Wüstenberg, T., Scheich, H., and Heinze, H. J. (2002). Phonetic Perception and the Temporal Cortex. Neurolmage 15, 733-746.

Jones, J. A., and Callan, D. E. (2003). Brain activity during audiovisual speech perception: An fMRI study of the McGurk effect. Neuroreport 14, 1129-1133.

Kaas, J. H., and Hackett, T. A. (2000). Subdivisions of auditory cortex and processing streams in primates. Proceedings of the National Academy of Sciences 97, 11793-11799.

Kim, J.-J., Crespo-Facorro, B., Andreasen, N. C., O'Leary, D. S., Zhang, B., Harris, G., and Magnotta, V. A. (2000). An MRI-Based Parcellation Method for the Temporal Lobe. NeuroImage 11,271-288.

King, A. J., and Calvert, G. A. (2001). Multisensory integration: perceptual grouping by eye and ear. Current Biology 11, R322-R325.

Liberman, A. M. (1992). The relation of speech to reading and writing. In Orthography, Phonology, Morphology and Meaning, R. Frost, and L. Katz, eds. (Amsterdam, Elsevier Science Publishers B.V.).

Longcamp, M., Anton, J. L., Roth, M., and Velay, J. L. (2003). Visual presentation of single letters activates a premotor area involved in writing. Neurolmage $19,1492-1500$.

Macaluso, E., Frith, C. D., and Driver, J. (2000). Modulation of human visual cortex by crossmodal spatial attention. Science 289, 1206-1208.

Massaro, D. W., Cohen, M. M., and Thompson, L. A. (1988). Visible language in speech perception: lipreading and reading. visible language 22.

McGurk, H., and MacDonald, J. (1976). Hearing lips and seeing voices. nature 263, 747. 
Meredith, M. A. (2002). On the neuronal basis for multisensory convergence: a brief overview. Cognitive Brain Research 14, 31-40.

National Reading Council (1998). Preventing reading difficulties in young children (Washington, DC, National Academy Press).

Olson, I. R., Christopher Gatenby, J., and Gore, J. C. (2002). A comparison of bound and unbound audio-visual information processing in the human cerebral cortex. Cognitive Brain Research 14, 129-138.

Paulesu, E., Perani, D., Blasi, V., Silani, G., Borghese, N. A., De Giovanni, U., Sensolo, S., and Fazio, F. (2003). A Functional-Anatomical Model for Lipreading. Journal of Neurophysiology 90, 2005-2013.

Perfetti, C. A., and Sandak, R. (2000). Reading optimally builds on spoken language: Implications for deaf readers. Journal of Deaf Studies and Deaf Education 5, 32-50.

Polk, T., Stallcup, M., Aguirre, G., Alsop, D., D'Esposito, M., Detre, J., and Farrah, M. (2002). Neural Specialization for Letter Recognition. Journal of Cognitive Neuroscience 14, 145-159.

Raij, T., Uutela, K., and Hari, R. (2000). Audiovisual integration of letters in the human brain. Neuron 28, 617-625.

Sams, M., Aulanko, R., Hamalainen, M., Hari, R., Lounasmaa, O. V., Lu, S. T., and Simola, J. (1991). Seeing speech: visual information from lip movements modifies activity in the human auditory cortex. Neuroscience Letters $127,141-145$.

Schroeder, C. E., and Foxe, J. J. (2002). The timing and laminar profile of converging inputs to multisensory areas of the macaque neocortex. Cognitive Brain Research 14, 187-198.

Sekiyama, K., Kanno, I., Miura, S., and Sugita, Y. (2003). Auditory-visual speech perception examined by $\mathrm{AMRI}$ and PET. Neuroscience Research 47, 277-287.

Shah, N., Steinhoff, S., Mirzazade, S., Zafiris, O., Grosse-Ruyken, M. L., Jäncke, L., and Zilles, K. (2000). The Effect of Sequence Repeat Time on Auditory Cortex Stimulation During Phonetic Discrimination. Neurolmage 12, 100-108.

Sumby, W. H., and Pollack, 1. (1954). Visual contribution to speech intelligibility in noise. The Journal of the Acoustical Society of America 26, 212-215.

Suzuki, M., Kitano, H., Kitanishi, T., Itou, R., Shiino, A., Nishida, Y., Yazawa, Y., Ogawa, F., and Kitajima, K. (2002). Cortical and subcortical activation with monaural monosyllabic stimulation by functional MRI. Hearing Research 163, 37-45.

Tervaniemi, M., Medvedev, S. V., Alho, K., Pakhomov, S. V., Roudas, M. S., van Zuijen, T. L., and Näätänen, R. (2000). Lateralized automatic auditory processing of phonetic versus musical information: A PET study. Human Brain Mapping 10, 74-79.

Wallace, M. T., Wilkinson, L. K., and Stein, B. E. (1996). Representation and integration of multiple sensory inputs in primate superior colliculus. Journal of Neurophysiology 76, 1246-1266.

Wright, T. M., Pelphrey, K. A., Allison, T., McKeown, M. J., and McCarthy, G. (2003). Polysensory Interactions along Lateral Temporal Regions Evoked by Audiovisual Speech. Cerebral Cortex 13, 1034-1043. 


\section{Chapter 3}

\section{The effect of temporal asynchrony on the integration of letters and speech sounds}

This chapter is based on:

Van Atteveldt, N. M., Formisano, E., Blomert, L. and Goebel, R. The effect of temporal asynchrony on the multisensory integration of letters and speech sounds. In revision. 


\section{Abstract}

Temporal proximity is a critical determinant for cross-modal integration by multisensory neurons. Information content may serve as an additional binding factor for more complex or less natural multisensory information. Letters and speech sounds, which form the basis of literacy acquisition, are not naturally related but associated through explicit learning. We investigated the relative importance of temporal proximity and information content on the integration of letters and speech sounds by manipulating both factors within the same fMRI design. The results reveal significant interactions between temporal proximity and content congruency in anterior and posterior auditory association cortex, indicating that temporal synchrony is critical for the integration of letters and speech sounds. The temporal profiles for multisensory integration in the auditory association cortex resemble those demonstrated for single multisensory neurons in different brain structures and animal species. This similarity suggests that basic neural integration rules apply to the binding of multisensory information that is not naturally related, but overlearned during literacy acquisition. Furthermore, the present study shows the suitability of fMRI to study temporal aspects of multisensory neural processing. 


\section{Introduction}

In the natural environment, multisensory stimuli arising from the same event are in close temporal proximity. Not surprisingly, temporal correspondence is a key determinant for the binding of information from different modalities, as is demonstrated in multisensory neurons in the superior colliculus and cortex in the cat (Meredith et al., 1987; Stein and Wallace, 1996) as well as in primates (Wallace et al., 1996). In accordance to these neurophysiological data, the importance of temporal coincidence has also recently been demonstrated using high-resolution fMRI in the monkey auditory cortex (Kayser et al., 2005).

In these studies, simple transient stimuli such as light flashes and noise bursts are commonly used (for a review, see Stein and Meredith, 1993). When the complexity of multisensory information increases, information content of the unisensory inputs may serve as an additional binding factor (Calvert et al., 1998; Pourtois and de Gelder, 2002; Laurienti et al., 2004). Multisensory information may even be exclusively related by information content, e.g. when the unisensory inputs are not naturally related. Studies using complex natural multisensory materials that share information content in addition to temporal onset, such as audiovisual speech, have shown that a larger temporal disparity is allowed before integration is disrupted (Massaro and Cohen, 1993; Massaro et al., 1996; Munhall et al., 1996; Munhall and VatikiotisBateson, 2004). Taken together, the importance of temporal proximity seems to depend on the nature and complexity of the multisensory information. We investigated the role of temporal proximity on the integration of letters and speech sounds, which are not naturally related but explicitly learned during literacy acquisition, and therefore initially only related by information content.

In speech-based alphabetic scripts, letters and speech sounds are the basic elements of correspondence between written and spoken language. Therefore, learning the correspondences between the letters and speech sounds of a language is a crucial step in literacy acquisition (Ehri, 2005). In literate adults, letter-speech sound associations can be considered as overlearned paired associates. However, developmental dyslexics encounter problems learning the correspondences between letters and speech sounds, which is thought to be one of the main causes underlying their reading difficulties (Vellutino et al., 2004). Taken together, it is of great relevance to elucidate the role of temporal proximity in the neural binding of letters and speech sounds, both for a better understanding of the principles underlying multisensory integration in the human brain, as well as considering the important role of letter-sound correspondences in alphabetic literacy.

In a previous $\mathrm{AMRI}$ study, we demonstrated that heteromodal superior temporal regions (superior temporal gyrus and sulcus (STS/STG)) and modality-specific posterior auditory association cortex (planum temporale (PT)) are crucially involved in the neural binding of letters and speech sounds (Van Atteveldt et al., 2004 [chapter 2]). In the present study, we used fMRI to address the question how these multisensory effects in the auditory association cortex and heteromodal STS/STG are influenced by a temporal offset between the letters and speech sounds. For this purpose, we manipulated both the temporal relation (stimulus onset asynchrony, SOA) and content congruency (same/different identity) between letters and speech sounds within the same experimental design. 
As substantiated in recent methodological and review papers, multisensory fMRI results should be interpreted with caution (Calvert, 2001), especially when the criterion of super-additivity is used (Beauchamp, 2005b; Laurienti et al., 2005). One of the main reasons for this is that with fMRI, large amounts of neurons are sampled simultaneously, which complicates the inference of integrative operations on the neuronal level, and thereby the use of criteria derived from electrophysiological studies Another important reason is that because of the intrinsic nature of the BOLD response and its limited dynamic range, a super-additive response at the neuronal level is not necessarily reflected in a super-additive change of the BOLD FMRI signal.

We used a congruency effect (at different SOAs) to determine the influence of temporal relation on multisensory integration. In this analysis, two bimodal conditions are contrasted to each other, one in which the stimuli have the same identity (congruent) and one in which the stimuli are of different identity (incongruent). The congruency effect can be used as a criterion for multisensory integration, since a distinction between corresponding and non-corresponding letters and speech sounds can not be established unless the unisensory inputs have been integrated successfully. An important advantage of using the congruency effect is that it allows manipulation of the temporal relation between the bimodal stimuli within the same design. Interactions between temporal relation and congruency therefore directly demonstrate an influence of temporal relation on multisensory integration.

Regions exhibiting a congruency effect are not necessarily performing integrative operations themselves, as it can not be excluded that this effect may reflect feedback from a different region where integration takes place (Van Atteveldt et al., 2004 [chapter 2]). To gain more detailed insight in the functional properties of different regions involved in letter-speech sound integration, it is important to inspect unimodal responses in candidate integration regions (Wright et al., 2003; Beauchamp, 2005b). Therefore, we presented letters and speech sounds also unimodally. This enabled additional analyses using the criterion that bimodal responses should exceed both unimodal responses (Van Atteveldt et al., 2004 [chapter 2]). This criterion was termed the max criterion by Beauchamp (2005b).

In analogy to electrophysiological studies (Meredith and Stein, 1983; Meredith et al., 1987; Stein and Wallace, 1996; Wallace et al., 1996), we visualized the magnitude of multisensory interaction (MSI) at different SOAs in regions of interest (ROIs) revealed by the congruency $x$ SOA interaction and the max criterion. In electrophysiology, MSI has been defined as a significant difference between the number of impulses evoked by a multisensory stimulus and the number of impulses evoked by the most effective unisensory stimulus, which can either be an enhancement or depression (Stein et al., 2004). Although the nature of the measured signal in the present study is evidently different, the same definition is conceptually attractive to quantify and visualize the effect of SOA on multisensory fMRI responses. 


\section{Results}

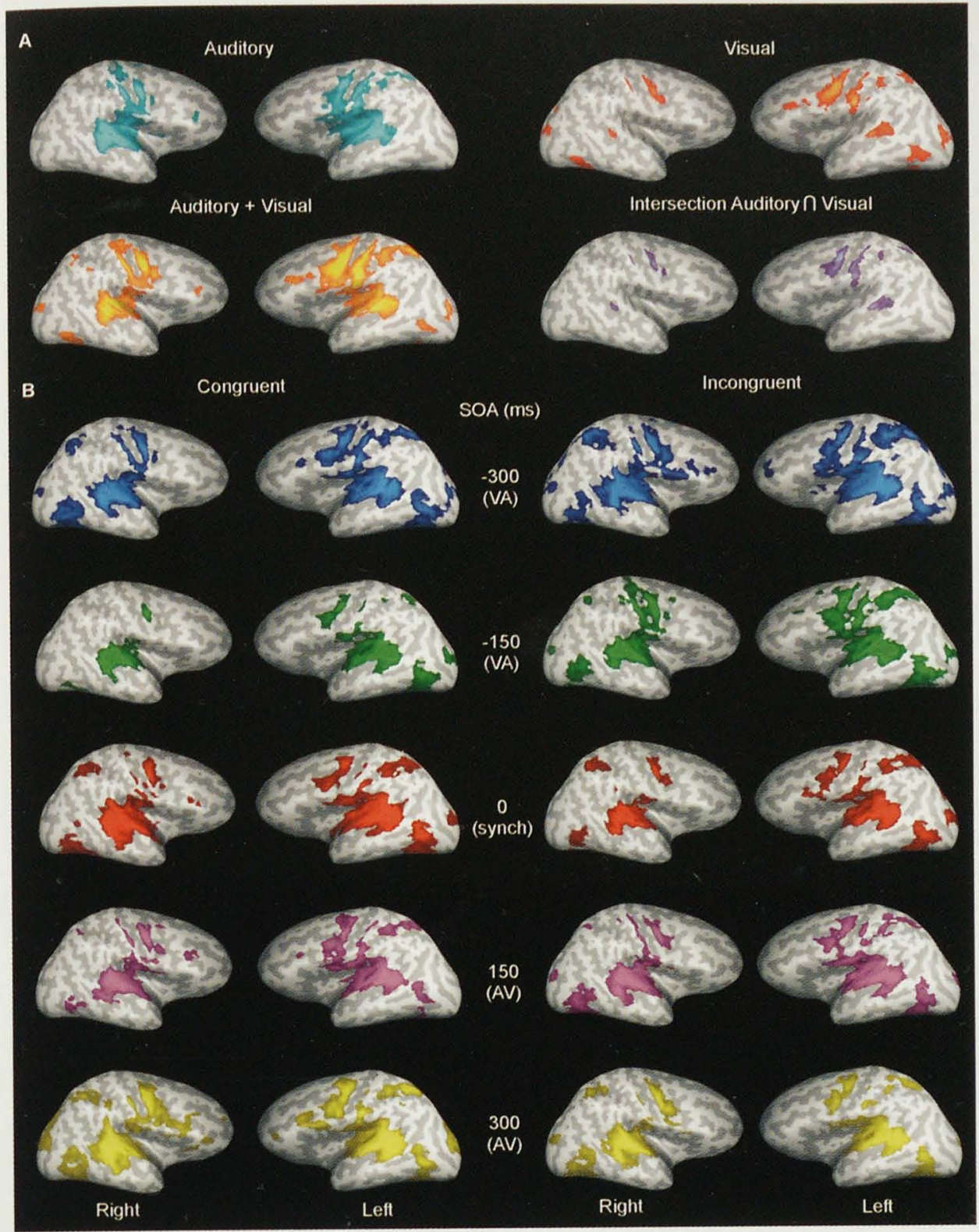

Figure 3.1. Overview of activation patterns for unimodal and bimodal presentation of letters and speech sounds. 
Figure 3.1 (Previous page). Overview of activation panterns for unimodal and bimodal presentation of letters and speech sounds.

(A) Multisubject general linear model (GLMI) maps of the unimodal predictors against baseline (upper. row, left: [Audirory > Baseline], right: [Visual > Baseline]), the combined unimodal predictors (lower row, left: [(Auditory + Visual) $>$ Baseline]) and the intersection of both umimodal predictors against baseline (lower row, right: [Visual > Baseline] $\cap$ [Auditory $>$ Baseline]).

(B) Multisubject general linear model (GLMI) maps of the bimodal predictors against baseline for the five different stimulus onset asynchronies (SOAs): -300 (blue map), -150 (green map), 0 (red map), 150 (violet map), and 300 (yellow map). Negative SOAs indicate that the letter was presented first (VA), positive SOAs that the sounds were presented first $(A V)$. At SOA =0. letters and speech sounds were presented in synchrony (synch). The maps were created from corter-based aligned functional data and shown on the inflated cortical sheet of the individual brain used as target for the alignment.

\section{Overview of activated brain regions}

Figure 3.1 shows an overview of activated brain regions during the different unimodal (fig. 3.1A) and bimodal (fig. 3.1B) stimulation periods after cortex-based alignment of anatomical and functional data (see Materials and Methods). In the bimodal conditions, 5 different SOAs were used (stimulus onset asynchrony between the letter and speech sound). Negative SOAs indicate that the letter was presented first (VA), positive SOAs that the sounds were presented first $(A V)$. At $S O A=0$, letters and speech sounds were presented in synchrony (synch).

Figure 3.1 shows that letters and speech sounds activated similar occipital and temporal brain regions in all different conditions used in the present study. Furthermore, the occipital and temporal activations were consistent with our previous study (Van Atteveldt et al., 2004 [chapter 2]), and with other findings: single letters activated extrastriate lateral occipital cortex (e.g. Longcamp et al., 2003; Flowers et al., 2004), speech sounds activated anterior as well as posterior superior temporal cortex (see Arnott et al., 2004; Scott, 2005). Interestingly, the maps for unimodally presented letters and speech sounds overlapped in the superior temporal sulcus (STS) (fig. 3.1A, intersection Auditory $\cap$ Visual), indicating multisensory convergence of letter and speech sound processing in this region.

In addition to occipital and temporal activations, activated areas were also observed in pre- and postcentral gyri and inferior parietal cortex, with comparable patterns for all unimodal and the bimodal conditions. The activation of the precentral gyrus was most prominent and consistent across conditions. Activation of premotor areas by passive listening to speech sounds is consistent with other findings (Wilson et al., 2004) and suggests an influence of articulatory features on speech perception. The premotor regions activated by passive viewing of single letters may correspond to Exner's area, which is thought to be the motor center of writing (Longcamp et al., 2003; Matsuo et al., 2003).

\section{Congruency contrast}

For synchronous presentation, activation of superior temporal cortex by congruent stimulation was increased compared to incongruent stimulation (see fig. 3.2). Interestingly, this difference was absent or less pronounced for the asynchronous conditions: only the contrast map at $\mathrm{SOA}=0$ revealed significant differences between congruent and incongruent stimulation in the superior temporal cortex (fig. 3.2A, 
orange activation map). The location (table 3.1, and fig. 3.2A-B) and response patterns (fig. 3.2C) of the posterior regions correspond to those observed in the planum temporale (PT) in our previous study [chapter 2]. In addition, we found a similar response pattern in anterior auditory association cortex bilaterally (anterior superior temporal plane, aSTP, fig. 3.2A-B). Individual analyses (congruency contrast at SOA = 0 using GLMl) revealed PT regions in 7/8 subjects in the left hemisphere (average Talairach coordinates \pm s.e.m.: $-58 \pm 2,-29 \pm 4,15 \pm 1$ ) and in $7 / 8$ subjects in the right hemisphere $(61 \pm 1,-24 \pm 3,15 \pm 2)$; aSTP regions in $8 / 8$ subjects in the left hemisphere (average Talairach coordinates \pm s.e.m.: $-56 \pm 2,-8 \pm 1,6 \pm 1$ ) and in $7 / 8$ subjects in the right hemisphere $(58 \pm 2,-8 \pm 2,3 \pm 2)$.

The averaged $\mathrm{BOLD}$ response time-courses in figure $3.2 \mathrm{C}$ indicate that in the PT as well as in the aSTP, the response to congruent letter-sound pairs was stronger than to speech sounds presented in isolation, while the response to incongruent letter-sound pairs was weaker than to isolated speech sounds. This observation was confirmed by ROI-GLM analyses for Congruent > Auditory in right PT $(p<0.005)$, left aSTP ( $<<$ $0.005)$ and right aSTP $(\mathrm{p}<0.01)$ and marginally in left PT $(\mathrm{p}<0.1)$. ROI-GLM results of the Auditory $>$ Incongruent contrast was only significant in left PT and right aSTP $(p<0.05)$, approaching significance in the left aSTP $(p<0.1)$, and not significant in the right PT $(\mathrm{p}=0.2)$.

Table 3.1. Details of the regions of interest (ROIs) selected by the different analyses

\begin{tabular}{|c|c|c|c|c|c|c|}
\hline \multirow[t]{2}{*}{$\mathrm{ROI}$} & \multirow{2}{*}{$\begin{array}{l}\text { Hemi- } \\
\text { sphere }\end{array}$} & \multirow[t]{2}{*}{ Talairach } & \multirow{2}{*}{$\begin{array}{l}\mathrm{Nr} \text { of } \\
\text { voxels }\end{array}$} & \multicolumn{2}{|c|}{ Effect size* } & \multirow[t]{2}{*}{ Statistical test** } \\
\hline & & & & $T$ & $P$ & \\
\hline \multirow[t]{2}{*}{ PT } & Left & $-52,-31,15$ & 36 & 3.42 & 0.0007 & \multirow[t]{4}{*}{ Cong > Incong*** } \\
\hline & Right & $60,-20,16$ & 167 & 3.74 & 0.0004 & \\
\hline \multirow[t]{2}{*}{ aSTP } & Left & $-55,-8,7$ & 127 & 3.61 & 0.0005 & \\
\hline & Right & $64,-9,6$ & 139 & 4.04 & 0.0002 & \\
\hline \multirow[t]{2}{*}{ PT } & Left & $-54,-31,16$ & 13 & 3.35 & 0.0008 & \multirow{4}{*}{$\begin{array}{l}\text { Interaction SOA x } \\
\text { Congruency }\end{array}$} \\
\hline & Right & $60,-20,15$ & 101 & 3.59 & 0.0004 & \\
\hline \multirow[t]{2}{*}{ aSTP } & Left & $-56,-7,7$ & 189 & 3.72 & 0.0003 & \\
\hline & Right & $63,-10,6$ & 194 & 4.22 & 0.0002 & \\
\hline STS & Left & $-55,-43,13$ & 11 & 3.47 & 0.0005 & $\begin{array}{l}\text { (Bimodal > Unimodal) } \\
\cap(\text { Unimodal }>0)^{* * *}\end{array}$ \\
\hline
\end{tabular}

*Effect size $=$ average t-value and p-value for all voxels in the $R O I$

**Statistical test used for ROI selection (maps thresholded at $q(F D R)<0.05$ )

***These tests were performed at SOA $=0$ 


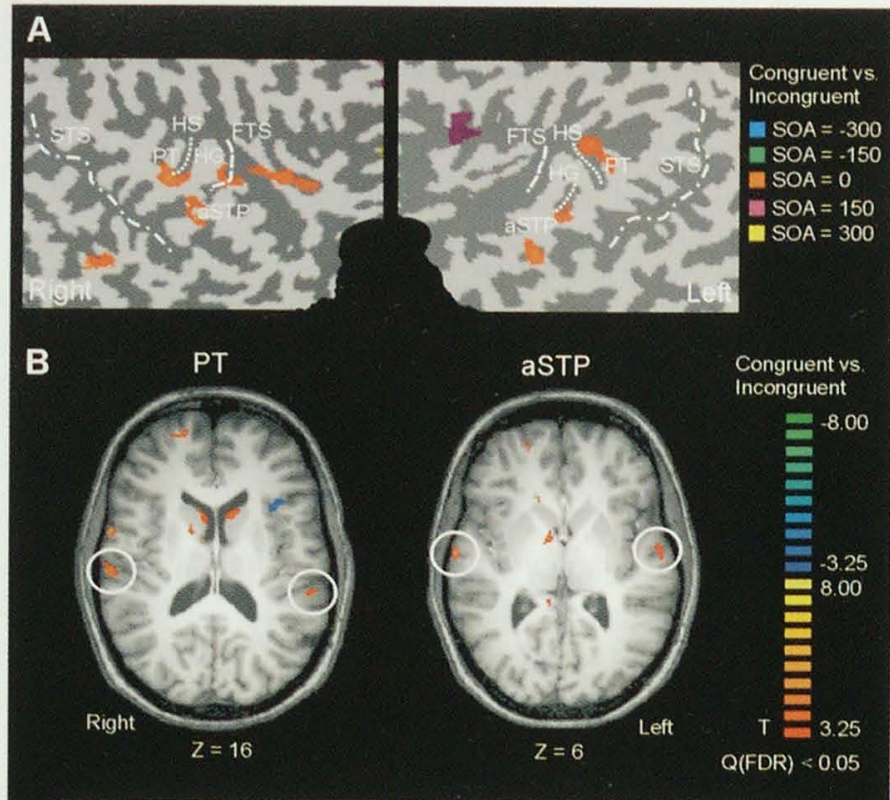

Figure 3.2. Results of the congruency contrast at all SOAS.

(A) Multisubject general linear model (GLMI) map of the congruency effects at different SOAs, created from cortex-based aligned functional data. Maps are shown on the flattened cortical sheet of the superior temporal lobe of the individual brain used as target for the cortex-based alignment. A congruency effect in superior temporal cortex was only found for synchronous stimuli (SOA $=0$ ), in PT and aSTP.

(B) Multisubject general linear model (GLMI) map of the congruency effect at SOA $=0$ shown in transversal slices. A significant congruency effect in superior temporal cortex was observed in PT and aSTP bilaterally (white circles).

(C) Averaged time-courses of the BOLD response (in percent signal change) in the PT and aSTP during unimodal (visual, red lines; auditory, blue lines) and bimodal (congruent, violet lines; incongruent, green lines) synchronous stimulation periods. Error bars indicate s.e.m. STS, Superior Temporal Sulcus; PT, Planum Temporale; $H S$, Heschl's Sulcus; HG, Heschl's gyrus; FTS, First Transverse Temporal Sulcus; ASTP, anterior. Superior Temporal Plane. 
A

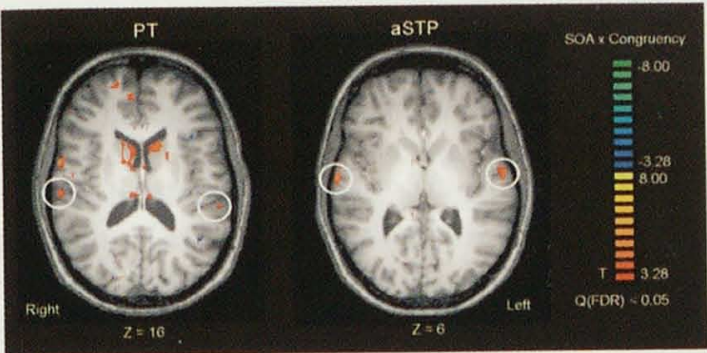

B
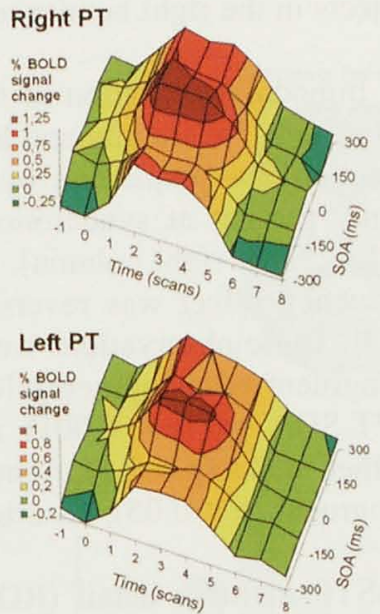

Right aSTP

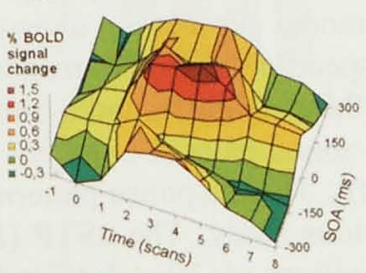

Left aSTP

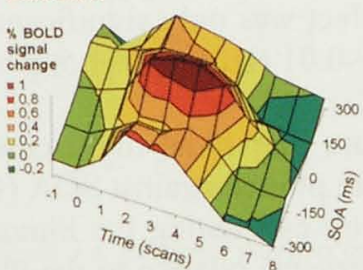

Incongruent
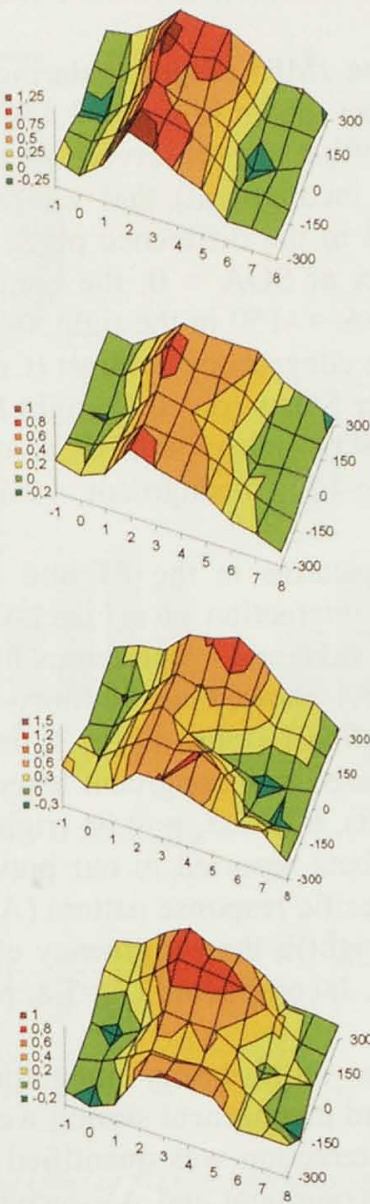

Congruent - Incongruent
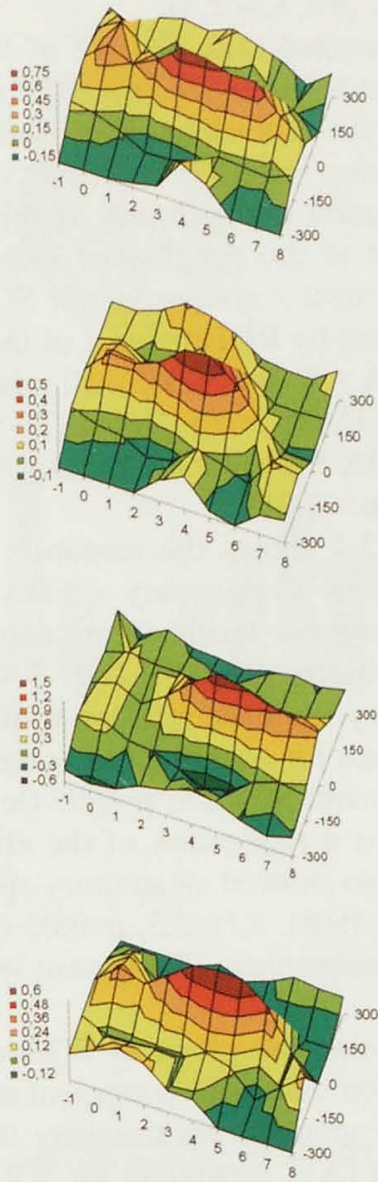

Figure 3.3. Results of the interaction analysis [Congruency $x$ SOA].

(A) Multisubject factorial general linear model map (GLM2) showing the interaction of SOA and Congruency in transversal slices.

(B) Averaged time-courses of the BOLD response (in percent signal change, indicated by the color coding on the $y$-axis) in the PT and aSTP bilaterally for all stimulus onset asynchronies (SOAs, plotted on the z-axis), plotted separately for congruent (left) and incongruent (middle) bimodal stimulation, and the difference between congruent and incongruent (right). The stimulation starts at time $=0$. 


\section{Interaction analysis}

Analysis of the fMRI time-series using a $2 \times 5$ factorial model (GLM2, see Materials and Methods) revealed significant interactions between Congruency and SOA in posterior (PT) and anterior (aSTP) auditory association cortex bilaterally (fig. 3.3A). These regions were identical to those revealed by the congruency contrast at $\mathrm{SOA}=0$ (see table 3.1). Individual analyses using GLM2 revealed a significant Congruency $x$ SOA interaction in PT $8 / 8$ subjects in the left hemisphere (average Talairach coordinates \pm s.e.m.: $-56 \pm 3,-31 \pm 4,14 \pm 1$ ), and in 6/8 subjects in the right hemisphere $(61 \pm 1,-25 \pm 1,15 \pm 2)$. In aSTP, Individual analyses revealed a Congruency $x$ SOA interaction in $7 / 8$ subjects in the left hemisphere (average Talairach coordinates \pm s.e.m.: $-55 \pm 1,-8 \pm 1,5 \pm 1$ ), and in $6 / 8$ subjects in the right hemisphere $(63 \pm 1,-9 \pm 1,4 \pm 1)$.

The averaged time courses of the $\mathrm{PMRI}$ response during bimodal stimulation at the different SOAs in PT and aSTP are shown in figure 3.3B. In the PT bilaterally and left aSTP, the time-courses indicate that the observed interaction was explained by a congruency effect (congruent $>$ incongruent) that was only present at synchronous presentation (most clearly visible in the difference plots, fig. 3.3B, right column). In addition to the congruency effect at $\mathrm{SOA}=0$, the congruency effect was reversed (incongruent $>$ congruent) for SOA $=-150$ in the right aSTP. These observations were confirmed by ROI analyses of the congruency contrast (Congruent $>$ Incongruent): left PT SOA $=0(p<0.005)$, all other SOAs $(p>0.1)$; right PT SOA $=0(p<0.001)$, all other SOAs $(p>0.1)$; left aSTP SOA $=0(p<0.001)$, all other SOAs $(p>0.1)$; right a STP SOA $=0(p<0.001)$, SOA $=-150$ (Incongruent $>$ Congruent, $p<0.05)$, all other SOAs $(p>0.01)$.

Figure 3.4 shows the response patterns in the PT and aSTP in more detail (ROIs selected by Congruency $\times$ SOA interaction at $\mathrm{q}(\mathrm{FDR})<0.05$ ). The bar graphs show fMRI response levels during unimodal and synchronous bimodal stimulation averaged over subjects. The PT (fig. 3.4A) showed an auditory-specific unimodal response (Auditory vs. Visual: $t(7)=6.6, p<0.001$ (left), $t(7)=5.3, p<0.001$ (right)), and a strong preference for congruent as compared to incongruent letter-sound pairs (Congruent vs. Incongruent: $t(7)=2.9, p<0.05$ (left), $t(7)=2.3, p<0.05$ (right)). This response pattern in the PT is a replication of the effects reported in our previous study. The aSTP (fig. 3.4B) also showed an auditory-specific response pattern (Auditory vs. Visual: $t(7)=4.3$, $p<0.005$ (left), $t(7)=2.5, p<0.05$ (right)), the congruency effect was only significant in the left hemisphere (Congruent vs. Incongruent: $t(7)=3.8, p<0.01$ (left), $t(7)=1.5, p>0.1$ (right)).

To examine the effect of SOA on multisensory integration, individual multisensory interaction values for congruent and incongruent stimuli were plotted against SOA (fig. 3.4, line graphs). Multisensory interaction was quantified by calculating the bimodal response ( $\mathrm{AV}$, separately for $\mathrm{AV}$-congruent and $\mathrm{AV}$-incongruent) relative to the most effective unimodal response $\left([\mathrm{A}, \mathrm{V}]_{\max }\right)$ in each individual subject ((AV$\left.\left.[\mathrm{A}, \mathrm{V}]_{\max }\right) /[\mathrm{A}, \mathrm{V}]_{\max } * 100 \%\right)$, see Materials and Methods). Therefore, the terms response enhancement (positive interaction) and response depression (negative interaction) in the following refer to the bimodal response relative to the most effective unimodal response (and not relative to the baseline response). In accordance to figure 3.3B, figure $3.4 \mathrm{~A}$ reveals that in the PT, the difference in multisensory interaction produced by congruent (response enhancement) and incongruent (response depression) stimulus 
pairs was only observed for synchronous presentation. The same effect of SOA on multisensory interaction was demonstrated for the aSTP (fig. 3.4B), although in this region the congruency effect at $\mathrm{SOA}=0$ was mainly due to an enhancement for congruent stimuli, without a response depression for incongruent stimuli. As already indicated by the time-courses (fig. 3.3B), an interesting different effect of SOA was observed in the right aSTP (fig. 3.4B). In this region, the congruency effect at SOA $=0$ (congruent $>$ incongruent) was reversed at $\mathrm{SOA}=-150$ (incongruent $>$ congruent). The response depression at this SOA was only present for congruent stimuli, indicating that the response evoked by a speech sound in this region is weaker when preceded by a visual letter of the same identity, but not when preceded by a different visual letter.

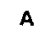

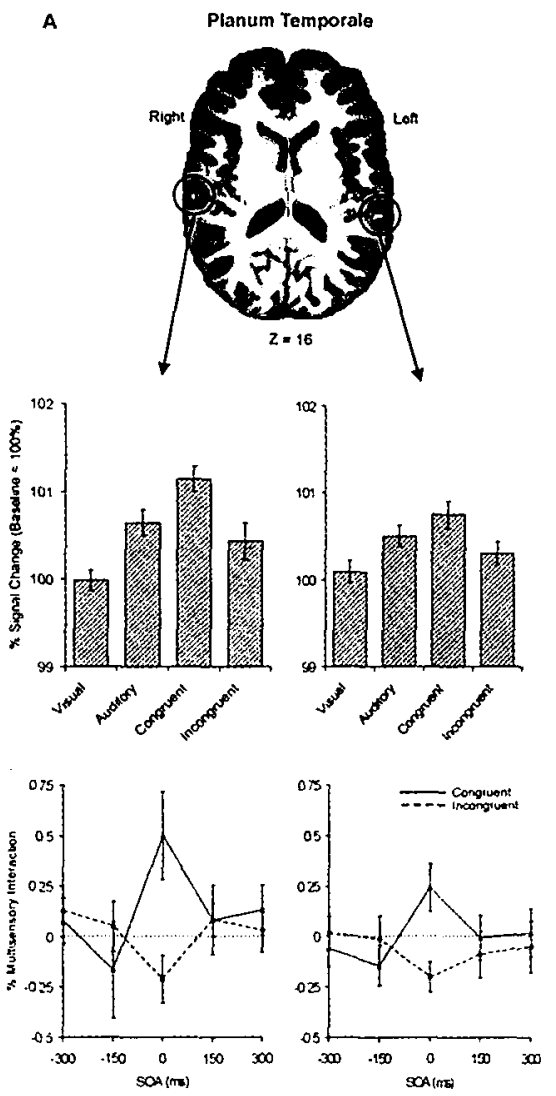
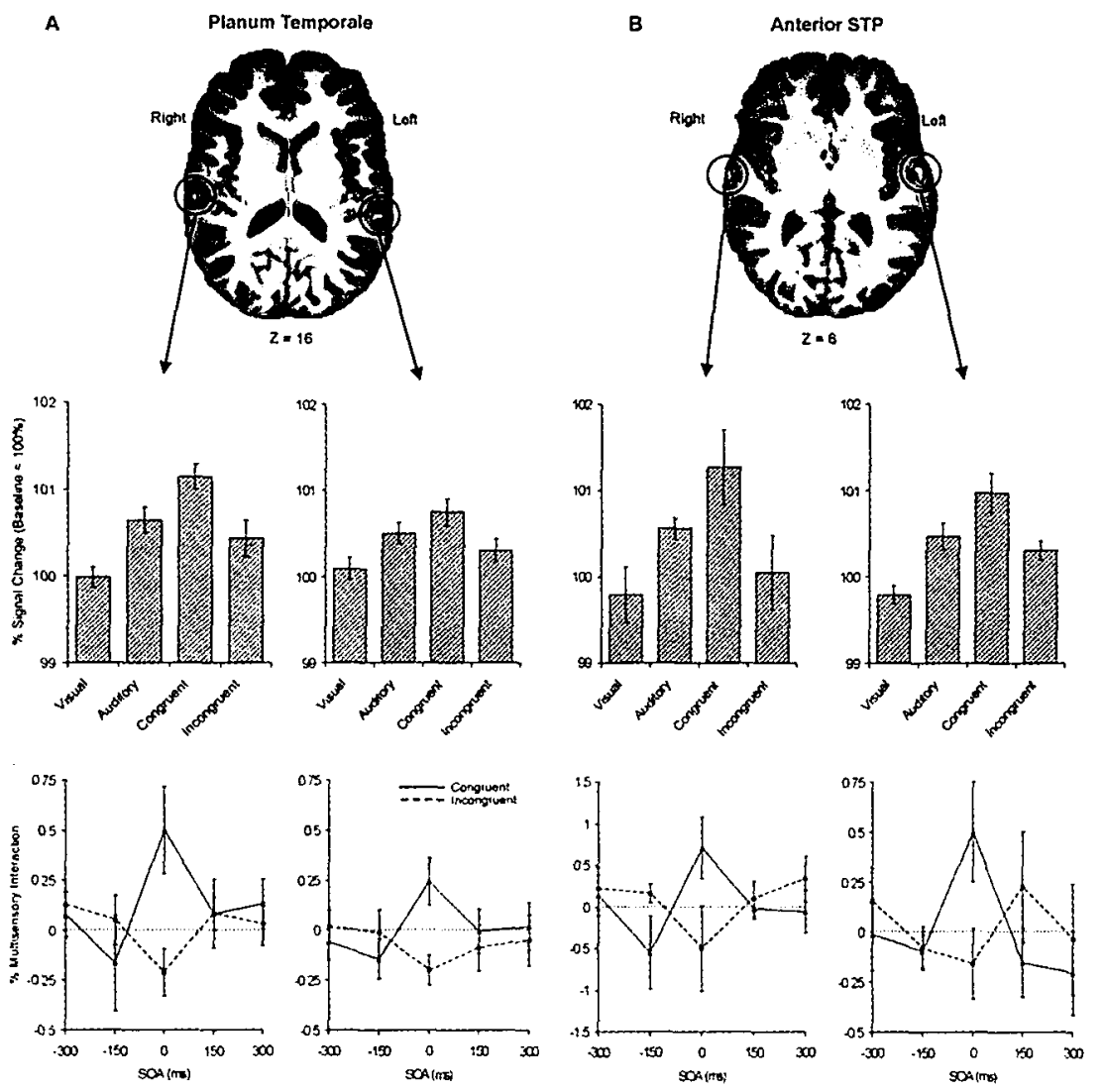

Figure 3.4. Response patterns and effect of SOA on multisensory interactions in PT (A) and aSTP (B).

Bar graphs: MR signal levels for the unimodal and bimodal synchronous conditions, averaged over subjects (error bars indicate s.e.m.). For each subject individually, the average MR signal during fixation periods (baseline) was set at 100\%.

Line graphs: Multisensory interaction (MSI) for congruent (solid lines) and incongruent (dashed lines) bimodal stimulation plotled as a function of SOA. MSI is defined as the bimodal response as percentage of the most effective unimodal response, and was calculated for each subject at each condition using the MR signal values plotted in the corresponding bar graphs. Error bars indicate variability across subjects (s.e.m.). 


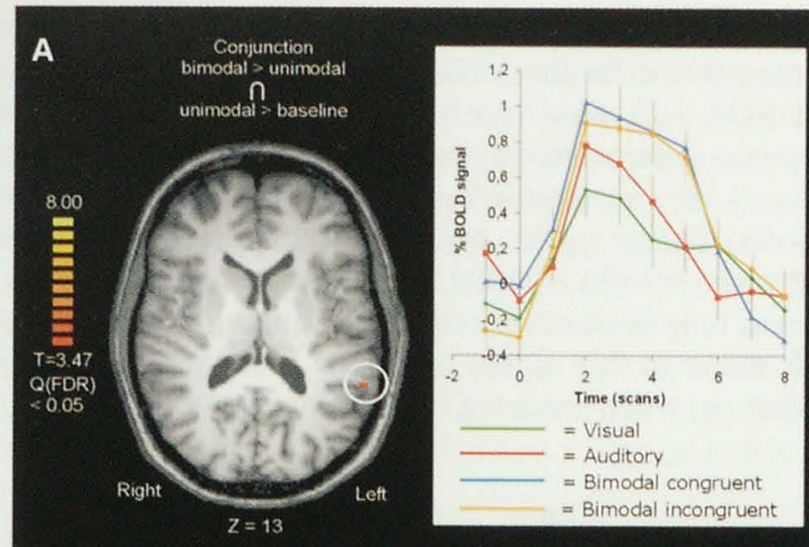

Figure 3.5. Results of the $\max$ criterion analysis [Bimodal > Unimodal] $\cap$ [Unimodal > baseline].

(A) Results of the max criterion analysis at $S O A=0$ shown in a transversal slice and the corresponding averaged timecourse of the BOLD response (visual, red lines; auditory, blue lines; congruent, violet lines; incongruent, green lines).

(B) Results of the max criterion analysis for all SOAs performed on cortex-based aligned data and shown on the cortical surface of the individual brain used as target for the alignment.

(C) bar graph: MR signal levels for the umimodal and bimodal synchronous conditions in the lefi STS, averaged over subjects (error bars indicate s.e.m.). Line graph: Multisensory interaction (MSI) for congruent (solid lines) and incongruent (dashed lines) bimodal stimulation plotted as a function of SOA. MSI is defined as the bimodal response as percentage of the most effective unimodal response, and was calculated for each subject at each condition using the MR signal values plotted in the corresponding bar graphs. Error bars indicate variability across subjects (s.e.m.). 


\section{Superior temporal sulcus}

The interaction analysis (SOA x Congruency) did not reveal regions in the STS. The superior temporal sulcus (STS) has been reported to be involved in letter-speech sound integration (Raij et al., 2000; Hashimoto and Sakai, 2004; Van Atteveldt et al., 2004 [chapter 2]), and in integration of other types of complex audiovisual information (see e.g. Beauchamp, 2005a). We explored the effect of SOA in the STS using the max criterion (the conjunction of [bimodal $>$ unimodal $\cap$ unimodal $>$ baseline], see Materials and Methods) at all SOAs. Figure 3.5A shows the result of the max criterion analysis at $\mathrm{SOA}=0$, which revealed a region in left STS (see also table 3.1). Note that this map corresponds to the regions shown in figure 3.1A, lower right (intersection Auditory $\cap$ Visual) for which it is also true that the response to bimodal stimulation is stronger that the response to unimodal stimulation. From the regions shown in this intersection map, only the left STS region passed this additional criterion. The response pattern in the left STS, shown by the BOLD response time courses in figure 3.5A, is a replication of the pattern found in our previous study (Van Atteveldt et al., 2004 [chapter 2]).

Figure 3.5C shows $\mathrm{AMRI}$ response levels for the unimodal and bimodal synchronous conditions in the left STS averaged over subjects (bar graphs), and the corresponding multisensory interaction values (line graph). The response pattern shown in the bar graph indicates that the enhanced response for bimodal stimulation was significant across subjects (Congruent vs. Auditory: $t(7)=3.2, p<0.05$; Congruent vs. Visual: $\mathrm{t}(7)=3.4, \mathrm{p}<0.01$; Incongruent vs. Auditory: $\mathrm{t}(7)=3.3, \mathrm{p}<0.05$; Incongruent vs. Visual: $\mathrm{t}(7)=3.7, \mathrm{p}<0.01)$. In contrast to the auditory-specific response pattern in the PT and aSTP, the STS showed a heteromodal response pattern (Auditory vs. Visual, $t(7)=0.9$, $\mathrm{p}=0.4$ ), indicating multisensory convergence. In addition, no congruency effect was observed in the STS (Congruent vs. Incongruent, $t(7)=0.5, p=0.6$ ).

The max criterion analysis revealed a similar region in left STS for all SOAs (fig. $3.5 \mathrm{~B}$ ), which indicates that a temporal offset between letters and speech sounds did not have an effect in the STS similar to that demonstrated for the auditory association cortex. This observation was confirmed by the multisensory interactions (fig. 3.5C, line graphs): significant positive multisensory interactions were observed for both bimodal conditions at all SOAs (except for at SOA $=-150$ (congruent) and SOA $=150$ (incongruent)).

\section{Discussion}

The principle aim of the present study was to elucidate the effect of temporal asynchrony on the neural integration of letters and speech sounds. We manipulated both the temporal relation (stimulus onset asynchrony, SOA) and content congruency (same/different identity) between letters and speech sounds within the same experimental design. Of particular interest for the present study are regions showing an interaction between SOA and content congruency when causing fMRI responses to letter-sound pairs, since such regions provide direct evidence for an influence of temporal relation on the neural binding of letters and speech sounds. The results clearly demonstrate that temporal relation and information content interact when causing fMRI 
responses to letter-speech sound pairs in anterior and posterior auditory association cortex (aSTP and PT), but not in the superior temporal sulcus (STS).

\section{Auditory association cortex}

One highly interesting observation is that temporal synchrony is a prerequisite for the occurrence of multisensory integration of letters and speech sounds in the posterior part of the auditory association cortex, the planum temporale (PT). The posterior part of the auditory cortex has been shown to play an important role in speech perception (e.g. Zatorre et al., 1992; Jäncke et al., 2002; Buchsbaum et al., 2005), and more specifically in the integration of written and spoken language (Nakada et al., 2001; Van Atteveldt et al., 2004 [chapter 2]). As is shown in figure 3.4A (line graphs), both the magnitude of response enhancement during congruent stimulation as well as the magnitude of response depression during incongruent stimulation, rapidly declined with temporal asynchrony. This observation implies that temporal correspondence overrules information content as binding factor, which is in accordance with predictions made by the time-window-of-integration (TWIN) model for multisensory integration (Colonius and Diederich, 2004; Diederich and Colonius, 2004). This model assumes that the time interval between the unisensory inputs acts like a filter by determining the probability of interaction. Other factors such as spatial configuration of the stimuli, and possibly also information content as suggested by the present results, have a subsequent role in determining the amount and direction (enhancement or depression) of interaction, once the temporal filter has been passed successfully. In the context of the present study, the dominance of temporal synchrony as determining factor for integration is a highly interesting finding since we studied multisensory associations that were initially only related by information content. This finding therefore supports the idea that basic neural integration rules apply to the binding of overlearned multisensory associations that are not naturally related.

Temporal relation and content congruency also interacted in the auditory association cortex anterior to the primary auditory cortex (aSTP). However, the effect of SOA in aSTP shows subtle differences from the effects observed in PT (line graphs in fig. 3.4). In the left aSTP, the congruency effect for synchronous stimuli is mainly due to an enhancement for congruent stimuli, without a depression for incongruent stimuli. Interestingly, in the right aSTP, the congruency effect was reversed when the visual stimulus preceded the auditory stimulus by $150 \mathrm{~ms}$ (SOA $=-150$, incongruent $>$ congruent). At this SOA, the response to congruent bimodal stimuli is weaker than the response to speech sounds presented alone (response depression) while the response to incongruent stimuli is not different from the unimodal response. The reduced fMRI response to speech sounds preceded by visually presented letters of the same identity might be explained by a cross-modal repetition suppression (Henson, 2003) or fMR adaptation (Grill-Spector and Malach, 2001) effect. Reduction of the fMR signal by repeated presentation of a single stimulus has been demonstrated within modalities and is thought to reflect neuronal adaptation. Although this interpretation is speculative at this point, fMR-adaptation designs may provide a way to gain insight in the functional characteristics of connections between different sensory systems in future research. By specifically tagging neuronal populations that are cross-modally activated, detailed investigation of the functional properties of these intersensory connections will be possible. 
The demonstrated effects of congruency in the auditory association cortex might alternatively be explained in terms of attention. Since we used a block design, subjects know from the first stimulus of a block whether all subsequent stimuli will be congruent or incongruent. This might lead to increased attention to the stimuli in the congruent blocks and decreased attention in the incongruent blocks, resulting in the observed response enhancement and depression. However, considering the high specificity of the congruency effect to focal regions in auditory association cortex, we think an explanation in terms of a general attention mechanism is unlikely, since this would predict an effect of congruency to be more widespread in the auditory cortex and to also include attention areas. Furthermore, attention alone can not explain why the congruency effect disappears, or even inverts (as observed in the right aSTP), when letters and sounds are asynchronously presented. Therefore, it seems plausible that the congruency effects in the auditory association cortex reflect (the result of) cross-modal integration. This is strongly supported by the characterization of multisensory integration by response enhancement and suppression in nonhuman electrophysiological studies (see Stein et al., 2004 for a review), and other human fMRI studies (Calvert et al., 2000; Saito et al., 2005).

The observed multisensory interaction effects in the auditory association cortex suggest that speech processing is influenced by visual orthographic information in focal regions anteriorly as well as posteriorly from the primary auditory cortex. Although the functional role of the anterior and posterior auditory processing streams is still under debate (Scott, 2005), (nonspatial) speech processing is reported in anterior as well as posterior superior temporal cortex (Arnott et al., 2004). The different temporal profile of multisensory interactions for both regions in the present study may suggest involvement in different aspects of letter-speech sound integration. The presumed cross-modal repetition suppression observed in the right aSTP may suggest a role in associating the exact identity of letters and speech sounds (the "what" pathway), while the PT may be involved in the "how" pathway, which is thought to be involved in sensory-motor integration of speech information (Buchsbaum et al., 2005; Scott, 2005). Consistent with the view of the planum temporale as "computational hub" (Griffiths and Warren, 2002) or sensory-motor interface (Buchsbaum et al., 2005; Scott, 2005), the PT might link sensory representations of letters and speech sounds with motor representations involved in speaking (Wilson et al., 2004) and writing (Longcamp et al., 2003). This view is supported by the activation of premotor cortex by the unimodally presented letters and speech sounds (figure 3.1 ).

\section{Superior Temporal Sulcus}

We found a heteromodal region in the left STS in which the bimodal response exceeded both unimodal responses, consistent with our previous study and with the assumed role of the STS in integration of letters and speech sounds (Raij et al., 2000; Hashimoto and Sakai, 2004; Van Atteveldt et al., 2004 [chapter 2]), and other types of audiovisual identity information (Calvert, 2001; Beauchamp et al., 2004; Amedi et al., 2005; Beauchamp, 2005a). Congruent and incongruent bimodal stimuli both evoked enhanced responses in the STS, which may seem unexpected considering the assumed integrative function. A possible explanation is that if congruency is determined in the STS, both congruent and incongruent combinations need computation and might therefore both lead to increased neural activity. This is in accordance to the fMRI study 
on complex audiovisual objects by Beauchamp and colleagues (2004) who also did not find a significant effect of congruency in the STS. In contrast to the present findings, Calvert et al. (2000) report an enhanced fMRI response for congruent and a depressed fMRI response for incongruent audiovisual speech. Other than design differences, this discrepancy might be related to the different nature and learning of audiovisual speech and letter-sound combinations (see also Van Atteveldt et al., 2004 [chapter 2]). While audiovisual speech occurs naturally and is learned early and implicitly (Kuhl and Meltzoff, 1982), letters are artificial and have to be associated with speech sounds by explicit instruction during literacy acquisition (Liberman, 1992). These differences might cause different computational demands during audiovisual integration in the STS. Using MEG, Raij et al. (2000) found differential interactions (although both negative) for congruent and incongruent audiovisual letters in the STS, which may seem contradictory to this interpretation. However, regarding the limited spatial resolution of MEG, the congruency effect in the study of Raij et al. may also have originated from slightly more superior temporal cortex, corresponding to the regions showing congruency effects in the present study (PT and aSTP).

Compared to the auditory association cortex, integration in the STS is less dependent on temporal synchrony (fig. 3.5), which is consistent with previous neuroimaging findings (Olson et al., 2002). Furthermore, the integration of audiovisual speech, which is thought to depend on integration in the STS (e.g. Calvert et al., 2000), has shown to be relatively unaffected by temporal disparity (Massaro and Cohen, 1993; Massaro et al., 1996; Munhall et al., 1996). Although integration in the left STS occurs within a wide temporal window in the present study, it appears to be least effective when the temporal offset between the visual and auditory stimuli is small (see fig. 3.5C).

\section{Implications for the neural mechanism of letter-speech sound integration}

Based on our findings, we propose the following neural mechanism of letter-speech sound integration (see also Van Atteveldt et al., 2004 [chapter 2]). Speech sounds are likely to be primarily represented and processed in the PT (Hickok and Poeppel, 2000; Griffiths and Warren, 2002). The next processing level, the STS, also receives visual information, and integrates both inputs within a broad range of SOAs. Depending on the temporal relationship between the inputs from both modalities, feedback regarding identity congruency is sent to the auditory association cortex, resulting in the observed temporal profiles of multisensory interaction there. A wider temporal window of integration in the STS enables a more flexible use of learned associations. It seems therefore plausible that the observed temporal windows for integration will be influenced by top-down strategic control when a task is introduced (Dijkstra et al., 1989). However, in the passive viewing and listening situation of the present study, basic rules of temporal proximity seem to apply to the automatic binding of letters and speech sounds, and feedback to the PT and left aSTP seems only to be provided when the stimuli are presented in temporal synchrony. Feedback to the right aSTP is also sent at short negative SOAs, and has the reversed effect on speech sound processing (depression for congruent subsequent stimuli), which may reflect cross-modal repetition suppression or adaptation. Furthermore, our data suggest that the STS sends feedback to aSTP and PT with different purposes: to aSTP for identification processes, 
and to PT for processes requiring sensory-motor integration. The PT may subsequently project to frontal and parietal regions involved in speech production and writing.

The response patterns and effects of temporal asynchrony observed in the auditory association cortex bears resemblance to those demonstrated for single multisensory neurons across brain areas and animal species (Meredith et al., 1987; Stein and Wallace, 1996; Wallace et al., 1996). This similarity suggests that multisensory neurons with similar properties exist in the human auditory association cortex, and thus that integration may take place directly there. Support for this suggestion is provided by the recent demonstration of integration of multisensory inputs in the auditory association cortex in macaques (Schroeder et al., 2001; Schroeder and Foxe, 2002), which has recently been demonstrated to be strongest for temporally coincident stimuli (Kayser et al., 2005). However, laminar input profiles indicated that visual input in the auditory cortex probably reflects feedback rather than direct input, possibly originating from the superior temporal polysensory area (STP) (Schroeder and Foxe, 2005), an area in the macaque likely corresponding to the upper bank of the human STS (Bruce et al., 1981). Furthermore, the PT and aSTP do not respond to visual unimodal stimulation (fig. $3.2 \mathrm{C}$ and fig. 3.4), while the STS shows multisensory convergence (fig. 3.5). Therefore, we think it is more plausible that the STS serves as an extra processing level where associations between letters and speech sounds are established, as was also indicated by our previous fMRI study (Van Atteveldt et al., 2004 [chapter 2]).

While audiovisual speech integration is known to be relatively unaffected by temporal asynchrony (Massaro and Cohen, 1993; Massaro et al., 1996; Munhall et al., 1996; Munhall and Vatikiotis-Bateson, 2004), the present study shows more stringent temporal constraints for the integration of letters and speech sounds. This apparent discrepancy may be explained by the fact that in audiovisual speech, the visual and auditory inputs share more features, e.g. time-varying aspects such as frequencyamplitude information (Munhall et al., 1996; Calvert et al., 1998; Munhall and Vatikiotis-Bateson, 1998; Amedi et al., 2005). Because letters and speech sounds lack these naturally corresponding features, it is tentative to assume that simultaneous onset is more critical for their integration. This idea bears resemblance to the finding of Dixon \& Spitz (1980) that asynchrony of audiovisual information with less concordant time-varying information (a hammer hitting a peg) is more easily detected than that of audiovisual speech.

\section{Conclusions}

In summary, multisensory integration of letters and speech sounds in the human auditory association cortex showed a strong dependency on the relative timing of the inputs. The critical role of input timing on multisensory integration has been demonstrated before at the neuronal level for naturally related visual and auditory signals. This similarity suggests that basic neural integration rules apply to the binding of multisensory information that is not naturally related, but overlearned during literacy acquisition. However, the mechanism by which the temporal constraints are effected may differ, that is, the temporal windows in the auditory association cortex observed in the present study may be the result of feedback from the STS. 


\section{Materials and Methods}

\section{Participants}

Eight healthy native Dutch subjects (7 female, mean age 23, range 19-29) participated in the present study. All subjects were university students enrolled in an undergraduate study program. Subjects without history of reading or other language problems were selected on the basis of a questionnaire. All subjects were right-handed, had normal or corrected-to-normal vision, and normal hearing capacity. Subjects gave informed written consent and were paid for their participation.

\section{Stimulation procedure}

Stimuli were speech sounds corresponding to single letters and their visually presented counterparts (vowels a, e, i, y, o, u, consonants d, g, h, k, l, n, p, r, s, t, z; vowels and consonants were presented in separate blocks). Speech sounds were digitally recorded (sampling rate $44.1 \mathrm{kHz}, 16$ bit quantization) from a female native Dutch speaker and represented isolated speech sounds (phonemes) rather than letter names. The selected speech sounds were recognized $100 \%$ correct in a pilot experiment $(n=10)$. Recordings were band-pass filtered $(180-10000 \mathrm{~Hz})$ and resampled at $22.05 \mathrm{kHz}$. Average duration of the speech sounds was $352( \pm 5) \mathrm{ms}$, the average sound intensity level was approximately $70 \mathrm{~dB}$ SPL. White lower case letters (typeface "Arial") were presented for $350 \mathrm{~ms}$ on a black background. During fixation periods and scanning, a white fixation cross was presented in the center of the screen.

A schematic description of the experimental design is shown in figure 3.6. Letters and speech sounds were presented in blocks of unimodal or bimodal stimulation. Congruency (congruent vs. incongruent) and temporal relationship (stimulus onset asynchrony, SOA) between the letters and speech sounds were systematically varied over the bimodal stimulation blocks. Five different SOAs were sampled: $-300,-150,0$, $150,300 \mathrm{~ms}$ (onset of the letter relative to onset of the sound). In total, there were 12 experimental conditions: unimodal visual, unimodal auditory, bimodal congruent at 5 SOAs, and bimodal incongruent at 5 SOAs. Subjects passively listened to and/or viewed the stimuli, to avoid interaction between activity related to stimulus processing and task-related activity due to cognitive factors.

To avoid interference of scanner noise with experimental auditory stimulation, stimuli were presented in silent delay periods between subsequent whole-brain scans (Eden $e t$ $a l ., 1999$ ) (see figure 3.6). Experimental blocks (24 s) were composed of 4 mini-blocks of 6 seconds each. One whole-brain scan was acquired in the beginning of each miniblock, during which only a fixation cross was presented. In the subsequent silent delay, 5 stimuli were presented with an inter trial interval (ITI) of $800 \mathrm{~ms}$. Because stimulus perception is uncontaminated by scanner noise in the silent period between successive scans, this stimulation procedure is very suitable for studying auditory processing with fMRI (Jäncke et al., 2002; Van Atteveldt et al., 2004 [chapter 2]). Stimulus presentation was synchronized with the scanner pulses using the software package "Presentation" (http://neurobehavioralsystems.com). Four repetitions of each of the 12 conditions were distributed over four experimental runs, resulting in the presentation of 80 trials per condition. The order of the conditions was randomized within runs and counterbalanced across runs. Fixation periods were presented in the beginning and end of each run (36s), and between each experimental block (24s). 


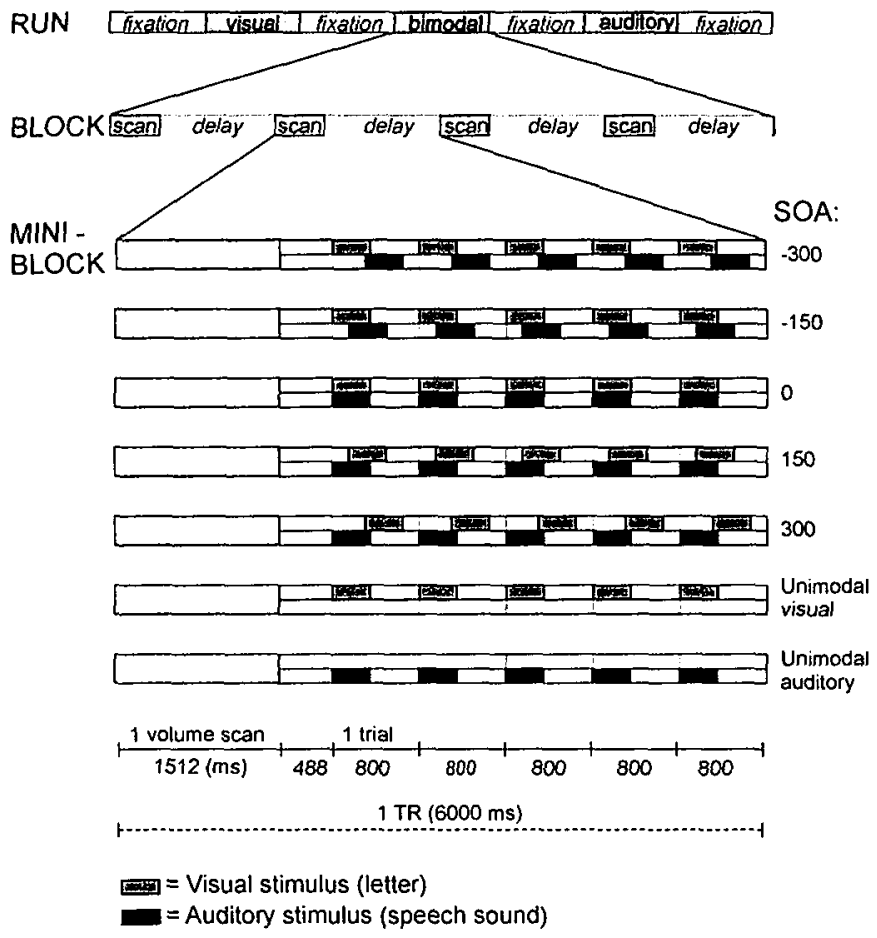

Figure 3.6.

Schematic description of the experimental design.

Experimental blocks of $24 \mathrm{~s}$ consisted of 4 mini-blocks of 6s. Each mini-block started with the acquisition of one whole-brain scan (1512 ms) followed by 5 experimental trials $(I T I=800 \mathrm{~ms})$ in a silent delay before the next scan was acquired. In the bimodal blocks, each trial consisted of $a$ visual and an auditory stimulus, which were presented with 5 different SOAs (one SOA per block). The timing details within one miniblock are depicted separately for each block type. TR, reperition time; SOA, stimulus onset asynchrony; ITI, inter trial interval.

\section{Scanning procedure}

Imaging was performed on a 3 Tesla whole-body system (Magnetom Trio, Siemens Medical Systems, Erlangen, Germany). In each subject, 4 runs of 104 volumes were acquired using a BOLD-sensitive EPI sequence (matrix: $64 \times 64 \times 24$, voxel size: $3.5 \times 3.5 \times 4.5 \mathrm{~mm}^{3}$, FOV: $224 \mathrm{~mm}^{2}$, TE/TRslice $=32 / 63 \mathrm{~ms}, \mathrm{FA}=75^{\circ}$ ). Sequence scanning time was $1512 \mathrm{~ms}$, inter-scan gap was $4488 \mathrm{~ms}$, resulting in a TR (sequence repeat time) of $6000 \mathrm{~ms}$. A slab of 24 axial slices (slab thickness: $10.8 \mathrm{~cm}$ ) was positioned in each individual such that the whole brain was covered, based on anatomical information from a scout image of 7 sagittally oriented slices. A highresolution structural scan (voxel size: $1 \times 1 \times 1 \mathrm{~mm}^{3}$ ) was collected for each subject using a Tl-weighted 3D MP-RAGE sequence (Magnetization-Prepared Rapid Acquisition Gradient Echo, TR $=2.3 \mathrm{~s}, \mathrm{TE}=3.93 \mathrm{~ms}, 192$ sagittal slices).

\section{Analysis of fMRI time-series}

Functional and anatomical images were analyzed using BrainVoyager 2000 and BrainVoyager QX (Brain Innovation, Maastricht, The Netherlands). The following preprocessing steps were performed: slice scan time correction (using sinc interpolation), linear trend removal, temporal high pass filtering to remove lowfrequency nonlinear drifts of 3 or less cycles per time course, and 3D motion correction to detect and correct for small head movements by spatial alignment of all volumes to the first volume by rigid body transformations. Estimated translation and rotation 
parameters were inspected and never exceeded $1 \mathrm{~mm}$. Functional slices were coregistered to the anatomical volume using position parameters from the scanner and manual adjustments to obtain optimal fit, and transformed into Talairach space. No spatial smoothing was applied to the functional MRI data.

For visualization of the statistical maps, all individual brains were segmented at the gray/white matter boundary (using a semi-automatic procedure based on intensity values), and the cortical surfaces were reconstructed and inflated. To improve the spatial correspondence mapping between subjects' brains beyond Talairach space matching, the reconstructed cortices were aligned using curvature information reflecting the gyral/sulcal folding pattern (cortex-based alignment procedure, described in Van Atteveldt et al., 2004 [chapter 2]). Statistical maps shown in slices are all thresholded at $\mathrm{q}(\mathrm{FDR})<0,05$ (Genovese et al., 2002).

The $\mathrm{AMRI}$ time-series were analyzed using two differently specified multi-subject fixed-effects general linear models (GLMs). In the first GLM, all 12 conditions were modeled as separate predictors (GLM1). The second was a $2 \times 5$ factorial model with the factors Congruency (congruent, incongruent) and SOA (-300, -150, 0, 150, $300 \mathrm{~ms})$, including the interaction term (Congruency $\mathrm{x} \mathrm{SOA}$ ) and separate predictors for the two unimodal conditions (GLM2). Predictor time-courses were adjusted for the hemodynamic response delay by convolution with a hemodynamic response function (Boynton et al., 1996).

We used GLM 1 to contrast all conditions against baseline to create statistical maps of the areas activated by letters, speech sounds and their combined presentation (figure 3.1). Furthermore, we performed the contrasts [bimodal congruent $>$ bimodal incongruent] at all 5 SOAs using GLM1 (referred to as "congruency contrast" in the results section). Clusters for which the congruency contrast was significant (at $q(F D R)$ $<0.05$ ) were saved as ROIs (specified in table 3.1). A third analysis performed with GLMl is the conjunction of [(bimodal congruent $>$ unimodal auditory) $\cap$ (bimodal congruent $>$ unimodal visual) $\cap$ (unimodal auditory $>$ baseline) $\cap$ (unimodal visual $>$ baseline)] (referred to as "max criterion analysis" in the results section). In this conjunction analysis, a new statistical value was computed for each voxel as the minimum of the statistical values obtained from the four included contrasts (Van Atteveldt et al., 2004 [chapter 2]). Clusters for which this new statistical value was significant (at $\mathrm{q}(\mathrm{FDR})<0.05$ ) were saved as ROIs. GLM2 was used to reveal interactions between congruency and SOA (referred to as "interaction analysis" in the results section). Clusters that showed a significant interaction (at $\mathrm{q}(\mathrm{FDR})<0.05$ ) between Congruency and SOA were saved as ROIs. In addition, we performed the same GLM1 and GLM2 analyses in individual subjects. Individual ROIs were selected at a more liberal threshold $(\mathrm{p}<0.05)$.

In the ROIs selected on basis of the multi-subject analyses, we estimated individual MR signal levels during the experimental conditions as percentage of the average MR level during fixation periods (baseline). We used these \% signal values to visualize the response pattern at $\mathrm{SOA}=0$ to provide additional information about intersubject variability of the experimental effects. Furthermore, we used the estimated MR signal levels to calculate multisensory interaction (MSI) values, to quantify multisensory integration effects. The magnitude of multisensory interaction is calculated by the formula: $\left.\left(\left(\mathrm{AV}-[\mathrm{A}, \mathrm{V}]_{\max }\right) /[\mathrm{A}, \mathrm{V}]_{\max }\right)^{*} 100 \%\right)$, where $\mathrm{AV}$ is the bimodal response and $[\mathrm{A}$, $\mathrm{V}]_{\max }$ the most effective unimodal response (Meredith and Stein, 1983; Meredith et al., 
1987; Stein and Wallace, 1996; Wallace et al., 1996). We used the total \% signal values (baseline $(100 \%)+$ signal change, e.g. $101.4 \%$ ), for the calculations of the MSI instead of the \% signal change (e.g. 1.4\%), to avoid the MSI to reach extremely high values for occasionally very low (approaching 0 ) maximal unimodal responses.

\section{Acknowledgements}

This work was supported by grant $608 / 002 / 2005$ of the Dutch Board of Health Care Insurance (College voor Zorgverzekeringen) awarded to L.B. We thank Peter Hagoort for providing access to the facilities of the F.C Donders Centre and Paul Gaalman for his technical assistance. 


\section{References}

Amedi A, von Kriegstein K, Van Atteveldt NM, Beauchamp MS, Naumer MJ (2005) Functional imaging of human crossmodal identification and object recognition. Experimental Brain Research 166:559-571.

Arnott SR, Binns MA, Grady CL, Alain C (2004) Assessing the auditory dual-pathway model in humans. Neuroimage 22:401-408.

Beauchamp M (2005a) See me, hear me, touch me: multisensory integration in lateral occipitaltemporal cortex. Current Opinion in Neurobiology 15:1-9.

Beauchamp M (2005b) Statistical criteria in fMRI studies of multisensory integration. Neuroinformatics 3:93-113.

Beauchamp M, Lee K, Argall B, Martin A (2004) Integration of auditory and visual information about objects in superior temporal sulcus. Neuron 41:809-823.

Boynton GM, Engel SA, Glover GH, Heeger DJ (1996) Linear Systems Analysis of Functional Magnetic Resonance Imaging in Human V1. Journal of Neuroscience 16:4207-4241.

Bruce C, Desimone R, Gross CG (1981) Visual properties of neurons in a polysensory area in superior temporal sulcus of the macaque. Journal of Neurophysiology 46:369-384.

Buchsbaum BR, Olsen RK, Koch PF, Kohn P, Shane Kippenhan J, Faith Berman K (2005) Reading, hearing, and the planum temporale. Neurolmage 24:444-454.

Calvert GA (2001) Crossmodal Processing in the Human Brain: Insights from Functional Neuroimaging Studies. Cerebral Cortex 11:1110-1123.

Calvert GA, Brammer MJ, lversen SD (1998) Crossmodal identification. Trends in Cognitive Sciences 2:247-253.

Calvert GA, Campbell R, Brammer MJ (2000) Evidence from functional magnetic resonance imaging of crossmodal binding in the human heteromodal cortex. Current Biology 10:649-657.

Colonius H, Diederich A (2004) Multisensory Interaction in Saccadic Reaction Time: A TimeWindow-of-Integration Model. Journal of Cognitive Neuroscience 16:1000-1009.

Diederich A, Colonius H (2004) Modeling the time-course of multisensory interaction in manual and saccadic responses. In: The handbook of multisensory processes (Calvert GA, Spence $C$, Stein BE, eds.), pp. 395-408. Cambridge: The MIT Press.

Dijkstra A, Schreuder R, Frauenfelder UH (1989) Grapheme context effects on phonemic processing. Language and Speech 32:89-108.

Dixon NF, Spitz L (1980) The detection of auditory visual desynchrony. Perception 9:719-721.

Eden GF, Joseph JE, Brown HE, Brown CP, Zeffiro TA (1999) Utilizing hemodynamic delay and dispersion to detect $\mathrm{MRI}$ signal change without auditory interference: the behavior interleaved gradients technique. Magnetic Resonance in Medicine 41:13-20.

Ehri LC (2005) Development of sight word reading: phases and findings. In: The Science of Reading: A Handbook (Snowling MJ, Hulme C, eds.), pp. Oxford: Blackwell publishing.

Flowers DL, Jones K, Noble K, VanMeter J, Zeffiro TA, Wood FB, Eden GF (2004) Attention to single letters activates left extrastriate cortex. NeuroImage 21:829-839.

Genovese C, Lazar N, Nichols T (2002) Thresholding of statistical maps in functional neuroimaging using the false discovery rate. NeuroImage 15:870-878. 
Griffiths TD, Warren JD (2002) The planum temporale as a computational hub. Trends in Neuroscience 25:348-353.

Grill-Spector K, Malach R (2001) fMR-adaptation: a tool for studying the functional properties of human cortical neurons. Acta Psychologica 107:293-321.

Hashimoto R, Sakai KL (2004) Learning letters in adulthood: direct visualization of cortical plasticity for forming a new link between orthography and phonology. Neuron 42:311-322.

Henson R (2003) Neuroimaging studies of priming. Progress in Neurobiology 70:53-81.

Hickok G, Poeppel D (2000) Towards a functional neuroanatomy of speech perception. Trends in Cognitive Sciences 4:131-138.

Jäncke L, Wüstenberg T, Scheich H, Heinze HJ (2002) Phonetic Perception and the Temporal Cortex. Neurolmage 15:733-746.

Kayser C, Petkov C, Augath M, Logothetis NK (2005) Integration of touch and sound in auditory cortex. Neuron 48:373-384.

Kuhl PK, Meltzoff AN (1982) The bimodal perception of speech in infancy. Science 218:1138-1141.

Laurienti PJ, Kraft RA, Maldjian JA, Burdette JH, Wallace MT (2004) Semantic congruence is a critical factor in multisensory behavioral performance. Experimental Brain Research 158:405414.

Laurienti PJ, Perrault TJ, Stanford TR, Wallace MT, Stein BE (2005) On the use of superadditivity as a metric for characterizing multisensory integration in functional neuroimaging studies. Experimental Brain Research 1166:289-297.

Liberman AM (1992) The relation of speech to reading and writing. In: Orthography, Phonology, Morphology and Meaning (Frost R, Katz L, eds.), pp. Amsterdam: Elsevier Science Publishers B.V.

Longcamp M, Anton JL, Roth M, Velay JL (2003) Visual presentation of single letters activates a premotor area involved in writing. NeuroImage 19:1492-1500.

Massaro DW, Cohen MM (1993) Perceiving asynchronous bimodal speech in consonant-vowel and vowel syllables. Speech Communication 13:127-134.

Massaro DW, Cohen MM, Smeele PM (1996) Perception of asynchronous and conflicting visual and auditory speech. Journal of the Acoustical Society of America 100:1777-1786.

Matsuo K, Kato C, Sumiyoshi C, Toma K, Thuy DHD, Moriya T, Fukuyama H, Nakai T (2003) Discrimination of Exner's area and the frontal eye field in humans - functional magnetic resonance imaging during language and saccade tasks. Neuroscience letters 340:13-16.

Meredith MA, Nemitz JW, Stein BE (1987) Determinants of multisensory integration in superior colliculus neurons. I. Temporal factors. Journal of Neuroscience 7:3215-3229.

Meredith MA, Stein BE (1983) Interactions among converging sensory inputs in the superior colliculus. Science 221:389-391.

Munhall K, Gribble P, Sacco L, Ward M (1996) Temporal constraints on the McGurk effect. Perception \& Psychophysics 58:351-362.

Munhall K, Vatikiotis-Bateson E (1998) The moving face during speech communication. In: Hearing by eye II: advances in the psychology of speechreading and auditory-visual speech (Campbell $R$, ed. pp. 123-139. Hove: Psychology Press.

Munhall K, Vatikiotis-Bateson E (2004) Spatial and Temporal Constraints on Audiovisual Speech Perception. In: The Handbook of Multisensory Processes (Calvert GA, Spence C, Stein BE, eds.), pp. 177-188. Cambridge, MA: The MIT Press. 
Nakada T, Fujii Y, Yoneoka Y, Kwee IL (2001) Planum temporale: where spoken and written language meet. European Neurology 46:121-125.

Olson IR, Christopher Gatenby J, Gore JC (2002) A comparison of bound and unbound audio-visual information processing in the human cerebral cortex. Cognitive Brain Research 14:129-138.

Pourtois G, de Gelder B (2002) Semantic factors influence multisensory pairing: a transcranial magnetic stimulation study. Neuroreport 13:1567-1573.

Raij T, Uutela K, Hari R (2000) Audiovisual integration of letters in the human brain. Neuron 28:617625.

Saito D, Yoshimura K, Kochiyama T, Okada T, Honda M, Sadato N (2005) Cross-modal binding and activated attentional networks during audiovisual speech integration: a functional MRI study. Cerebral Cortex 5:1750-1760.

Schroeder CE, Foxe JJ (2002) The timing and laminar profile of converging inputs to multisensory areas of the macaque neocortex. Cognitive Brain Research 14:187-198.

Schroeder CE, Foxe JJ (2005) Multisensory contributions to low-level, 'unisensory' processing. Current Opinion in Neurobiology 15:1-5.

Schroeder CE, Lindsley RW, Specht C, Marcovici A, Smiley JF, Javitt DC (2001) Somatosensory Input to Auditory Association Cortex in the Macaque Monkey. Journal of Neurophysiology 85:1322-1327.

Scott SK (2005) Auditory processing - speech, space and auditory objects. Current Opinion in Neurobiology 15:197-201.

Stein BE, Jiang H, Stanford TR (2004) Multisensory integration in single neurons of the midbrain. In: The handbook of multisensory processes (Calvert GA, Spence C, Stein BE, eds.), pp. 243-264. Cambridge: The MIT Press.

Stein BE, Meredith MA (1993) The merging of the senses. Cambridge, MA: MIT Press.

Stein BE, Wallace MT (1996) Comparisons of cross-modality integration in midbrain and cortex. Progress in Brain Research 112:289-299.

Van Atteveldt N, Formisano E, Goebel R, Blomert L (2004) Integration of letters and speech sounds in the human brain. Neuron 43:271-282.

Vellutino FR, Fletcher JM, Snowling MJ, Scanlon DM (2004) Specific reading disability (dyslexia): what have we learned in the past four decades? Journal of Child Psychology and Psychiatry 45:2-40.

Wallace MT, Wilkinson LK, Stein BE (1996) Representation and integration of multiple sensory inputs in primate superior colliculus. Journal of Neurophysiology 76:1246-1266.

Wilson SM, Saygin AP, Sereno MI, Iacoboni M (2004) Listening to speech activates motor areas involved in speech production. Nature Neuroscience 7:701-702.

Wright TM, Pelphrey KA, Allison T, McKeown MJ, McCarthy G (2003) Polysensory Interactions along Lateral Temporal Regions Evoked by Audiovisual Speech. Cerebral Cortex 13:1034-1043.

Zatorre RJ, Evans AC, Meyer E, Gjedde A (1992) Lateralization of phonetic and pitch discrimination in speech processing. Science 256:846-849. 


\section{Chapter 4}

\section{Top-down task effects overrule automatic multisensory responses to letter-sound pairs in auditory association cortex}

This chapter is based on:

Van Atteveldt, N. M., Formisano, E., Goebel, R., and Blomert, L. Top-down task effects overrule automatic multisensory responses to letter-sound pairs in auditory association cortex. Submitted. 


\section{Abstract}

In alphabetic scripts, letters and speech sounds are the basic elements of correspondence between spoken and written language. Therefore, investigations of the mechanism by which the brain integrates letters and speech sounds increase our understanding of the neural basis of literacy. In two previous fMRI studies using blocked stimulus presentation and passive perception, we found a cross-modal modulation of the response to speech sounds in the auditory association cortex by letters, expressed as a response enhancement by congruent letters and suppression by incongruent letters. Interestingly, temporal proximity was critical for this effect of congruency to occur. In the present study, we used fMRI to investigate the effect of stimulus presentation mode (by comparing blocked and event-related experiments) and task instruction (by comparing passive and active versions of the same experiment) on the neural integration of letters and speech sounds. In the active version, subjects had to perform a same/different matching task. The principle findings are 1) a replication of the previous results on passive integration using event-related fMRI, and 2) the disappearance of the effects of congruency and temporal proximity in the auditory association cortex during active matching. The first finding excludes an explanation of the congruency effect by different attention levels due to the blocked stimulus presentation, and shows the suitability of an event-related fMRI design for studying letter-sound integration. The second finding indicates that the task demands overruled the passive/default multisensory responses to letters and speech sounds, most likely because the task changed the behavioral relevance of the stimuli. The source of this top-down task effect is suggested to be a frontal-parietal network that controls explicit matching of the overlearned associations between letters and speech sounds. 


\section{Introduction}

The current information society makes literacy proficiency indispensable for social and economic success (National Reading Council, 1998). Therefore, insight in the neural basis of literacy skills is of utmost importance. In speech-based alphabetic scripts, letters and speech sounds are the basic elements of correspondence between written and spoken language. Learning the correspondences between the letters and speech sounds of a language is therefore a crucial step in literacy acquisition (Ehri, 2005), and interestingly, seems to be one of the main causes underlying reading difficulties in developmental dyslexia (Vellutino et al., 2004). For these reasons, investigations of the mechanism by which letters and speech sounds are associated in the brain are essential for a better understanding of the neural basis of literacy acquisition.

In two previous fMRI studies, we elucidated the functional neuro-anatomical mechanism for the integration of letters and speech sounds in literate adults (Van Atteveldt et al., 2004 [chapter 2]), and the importance of temporal proximity for successful integration (Van Atteveldt et al., submitted [chapter 3]). The first study revealed that responses to speech sounds in a modality-specific region of the auditory association cortex were modified by simultaneously presented letters. This cross-modal modulation was expressed by enhancement of the response to speech sounds by congruent letters and suppression by incongruent letters (hereafter referred to as "congruency effect"). Furthermore, the first study revealed an enhanced response to bimodally presented letter-sound pairs relative to their unisensory components in heteromodal regions in the superior temporal sulcus/gyrus (STS/STG). The second study showed that temporal proximity was critical for the congruency effect to occur in the auditory association cortex, while the enhanced bimodal response in heteromodal STS/STG was observed over a wider temporal window.

The heteromodal response pattern of the STS/STG suggested an integrative role for the STS/STG, which conformed to other reports providing evidence for its importance in multisensory integration of identity information (Amedi et al., 2005; Beauchamp, 2005; Calvert, 2001). We interpreted the observed congruency effect in the auditory cortex as a feedback modulatory influence from the STS/STG, in accordance with other studies advocating a feedback mechanism for audiovisual integration of speech information (Calvert et al., 2000; King and Calvert, 2001). Since there were no active task demands in these two previous studies, no top-down task-related influences were exerted over the integration process. Therefore, the previous findings reflect a default or automatic/mandatory integration mechanism for the overlearned associations between letters and speech sounds.

The previous findings provide valuable information regarding the functional neuroanatomy of letter-speech sound integration. However, several questions remain unanswered. First, the congruency effect in the auditory cortex might be explained partly in terms of attention or arousal because of the blocked stimulus presentation (see e.g. Beauchamp, 2005). In a block design, subjects know after the first stimulus of a block whether all subsequent stimuli will be congruent or incongruent. Therefore, in principle, only the first stimuli need to be integrated to determine the congruency. After this, increased attention levels in the congruent blocks and decreased attention levels in the incongruent blocks might have resulted in the observed response enhancement and suppression. Although the established specificity of the congruency effect make an 
explanation in terms of a general attention mechanism unlikely (Van Atteveldt et al., submitted [chapter 3]), it can only be excluded when the congruency effect would be replicated with random stimulus presentation.

A second open question is the effect of different task instructions on the revealed default integration mechanism. In the two previous studies on letter-sound integration, subjects were instructed to carefully view/listen to the stimuli without performing any active task. This probably means that the incoming information is integrated based on stimulus-related properties only, i.e. identity congruency and temporal proximity. However, the use of learned associations between sensory events is likely to be flexible and not restricted to passive perception. This may especially be true for letters and speech sounds, which have to be associated during different kinds of reading and spelling activities. Not surprisingly, next to stimulus-related binding factors, top-down factors generated by task instructions also seem to have a clear effect on multisensory integration (Andersen et al., 2004).

To elucidate these open issues, the present fMRI study addressed the following research question: what are the effects of stimulus presentation mode and task-related factors on the neural mechanism for letter-speech sound integration? For this purpose, we changed the experimental design from blocked to random stimulus presentation and included an active task: subjects were instructed to perform a same/different matching task on letter-sound pairs that were presented simultaneously (study 1) or at different temporal offsets (study 2). The congruency effect in the auditory cortex was demonstrated to be a very robust finding (Van Atteveldt et al., 2004 [chapter 2]), and showed a strong effect of temporal asynchrony (Van Atteveldt et al., submitted [chapter 3]). For these reasons, we focused on the congruency effect in the present studies. The same/different matching task forces the integration process to be active/explicit while leaving stimulus presentation unchanged, so the congruency effect with and without active task demands can be compared directly. A more specific question in this context is whether the temporal windows of integration (Van Atteveldt et al., submitted [chapter 3]) will be widened when integration is explicit. This may be expected because during active matching, the bimodal information is functionally relevant independent of temporal offset. An operationally defined aim is therefore to explore whether the congruency effect in the auditory cortex, and its narrow temporal window of occurrence, is influenced by top-down task demands.

A systematic exploration of the effects of different experimental designs on the networks of brain areas involved in multisensory integration also has important implications from a more general perspective. As pointed out by de Gelder and Bertelson (2003), demands of laboratory tasks may contaminate automatic perceptual integration processes, which complicates the interpretation of experimental results. Neuroimaging is especially valuable in this perspective, because integration (on the neural level) can directly be compared between situations with and without active tasks demands, while pure behavioral studies of multisensory integration oblige the use of an active task. Still, neuroimaging studies on multisensory integration report substantial variability in recruited brain mechanisms. Next to differences in stimulus material and analytical strategy, this may be attributed to different experimental paradigms (Calvert, 2001), which underlines the value of a systematic exploration of the effects of different experimental designs. 
The change from blocked to random stimulus presentation leads to additional effects on the fMRI measurements unrelated to the present research questions. Random stimulus presentation entails the use of an event-related fMRI design. One important consequence of this is that in a "silent" scanning paradigm, the relative amount of acoustic noise produced by fast gradient switching during functional imaging ("scanner noise") will be increased. In such paradigms, stimuli are presented in silent intervals between subsequent volume scans, which minimizes the effects of scanner noise on experimentally evoked auditory activation (Jäncke et al., 2002; Van Atteveldt et al., 2004 [chapter 2]). In a block design, multiple stimuli can be presented in a silent interval, compared to only one stimulus in an event-related design. The resulting shorter silent interval in an event-related design increases the relative amount of scanner noise, which may saturate the hemodynamic response in auditory areas and thereby limit the range in which the fMRI signal can increase due to experimental auditory stimulation. A second important consequence of using an event-related fMRI design is a more general one. In the two previous studies using blocked stimulus presentation, a series of trials of one condition (20) presented in each block summed up into an extended signal change so that differences between conditions could be determined with considerable statistical power. In contrast, fMRI responses to single letters and speech sounds (and their combinations) will be averaged to identify differences between conditions in an event-related design (Donaldson and Buckner, 2001). Although it has been shown that detectable signal changes can be elicited by very short single events (Savoy et al., 1995), it is not sure how the reduction in stimulation duration will affect the relative signal changes to single letters and speech sounds when an event-related fMRI design would be employed.

The present report consists of two different studies. In study 1 , the effect of the stimulus presentation mode was investigated by systematically changing the design from passive blocked stimulus presentation, to passive event-related stimulus presentation, to an active event-related design in which the matching task was introduced. The intermediate design (passive event-related) had two goals: 1) testing the hypothesis that the congruency effect found in the previous studies might be explained by attention or arousal due to the blocked presentation, and 2) controlling for the effects of the different stimulus presentation mode unrelated to task effects (higher scanner noise and shorter stimulus duration). After accounting for these additional effects in study 1, the effect of top-down task-related factors on letter-speech sound integration and its temporal constraints could directly be studied in study 2 . In this second study, we scanned subjects performing the same/different matching task using an event-related design in which both congruency and temporal relation of letters and speech sounds were manipulated, and compared the results directly to the passive integration mechanism.

\section{Methods}

\section{Stimulus presentation}

The same stimulus material was used in study 1 and 2 . Stimuli were speech sounds corresponding to single letters and their visually presented counterparts (vowels a, e, i, $\mathrm{y}, \mathrm{o}, \mathrm{u}$, consonants $\mathrm{d}, \mathrm{g}, \mathrm{h}, \mathrm{k}, \mathrm{l}, \mathrm{n}, \mathrm{p}, \mathrm{r}, \mathrm{s}, \mathrm{t}, \mathrm{z}$ ). Speech sounds were digitally recorded 
(sampling rate $44.1 \mathrm{kHz}, 16$ bit quantization) from a female native Dutch speaker and represented isolated speech sounds (phonemes) rather than letter names. The selected speech sounds were recognized $100 \%$ correct in a pilot experiment $(n=10)$. Recordings were band-pass filtered $(180-10000 \mathrm{~Hz})$ and resampled at $22.05 \mathrm{kHz}$. Average duration of the speech sounds was $352( \pm 5) \mathrm{ms}$, the average sound intensity level was $71.3( \pm 0.2) \mathrm{dB}$. White lower case letters (typeface "Arial") were presented for $350 \mathrm{~ms}$ on a black background. During the fMRI experiments, the visual stimuli were projected (Sanyo PLC-XT16) onto a frosted screen positioned at the rear end of the MR scanner bore, and viewed by the participants through a mirror mounted onto the head coil. Stimulus presentation was synchronized with the scanner pulses using the software package "Presentation" (http://neurobehavioralsystems.com). Participants" responses were registered by a hand held fiber-optic response system (LUMItouch fMRI Optical Response keypad, Photon Control, Burnaby, Canada, www.photonixco.com).

\section{Study 1}

Participants: Six healthy native Dutch subjects (2 male, mean age 25, range 22-27) participated in the first fMRI experiment. All subjects were university students enrolled in an (under) graduate study program and were selected based on a questionnaire including questions concerning (present or history of) reading or other language problems. All were right-handed, had normal or corrected-to-normal vision, and normal hearing capacity. Subjects gave informed written consent and were paid for their participation.

Experimental procedure: In each subject, 3 different experiments were conducted: a passive experiment in which the stimuli were presented in blocks (passive block), a passive experiment in which the stimuli were presented randomly (passive eventrelated) and an active experiment in which the stimuli were presented randomly and a response was required to each stimulus (active event-related).

In the passive block runs (figure 4.1B) stimuli were presented in blocks of four different conditions: unimodal visual, unimodal auditory, bimodal congruent and bimodal incongruent. In the bimodal conditions, visual letters and speech sounds were presented simultaneously. Experimental blocks (20.8 s) were composed of 4 miniblocks of 5.2 seconds. In the first 1.5 seconds of each mini-block, one brain volume was acquired (see "scanning procedure"). No stimuli were presented in this period, only a fixation cross to keep the eyes of the subjects focused on the center of the screen. In the subsequent silent 3.7 seconds, 5 stimuli were presented. In each run, 2 blocks (each consisting of 20 stimuli) of each condition were presented. Fixation periods were presented in the beginning and end of each run (26s), and between each experimental block (20.8s). The order of the experimental blocks was pseudorandomized within runs and counterbalanced across runs.

In the passive event-related runs (figure $4.1 \mathrm{C}$, study 1), stimuli of the same four conditions (unimodal visual, unimodal auditory, bimodal congruent and bimodal incongruent) were presented randomly. In the bimodal trials, visual letters and speech sounds were presented simultaneously. The interval between two subsequent stimuli was 14,16 or 18 s (corresponding to 7,8 , or 9 TRs). Stimuli were presented in silent delay periods between subsequent volume scans (see "scanning procedure"). A fixation cross was presented on the screen between the stimuli. In each run, 10 trials of 
each condition were presented. In the active event-related runs (figure 4.1C, study 1), the same design was used as in the passive event-related runs, except that only bimodal stimuli were presented (congruent and incongruent). In each run, 20 trials of each condition were presented. Subjects were instructed to respond as fast and accurately as possible to the question: "Are the letter and the sound the same?" after each stimulus pair. Reaction times were recorded from the onset of the stimulus pair. The correct button (middle or index finger of the right hand) for "yes" and "no" was counterbalanced over the subjects.

Scanning procedure: In two of the subjects, imaging was performed on a 3 Tesla whole-body system (Magnetom Trio, Siemens Medical Systems, Erlangen, Germany) located at the F.C. Donders center in Nijmegen, The Netherlands. In four of the subjects, imaging was performed on a 3 Tesla head scanner (Magnetom Allegra, Siemens Medical Systems, Erlangen, Germany) located at the Maastricht Brain Imaging Centre (M-BIC) in Maastricht, The Netherlands. In each subject, 2-4 passive block runs of 70 volumes were acquired, followed by 2-4 passive event-related runs of 330 volumes, followed by 2 active event-related runs of 330 volumes. In four of the subjects, all runs were acquired in the same session. In two of the subjects, the passive block runs were acquired in an earlier session.

A BOLD-sensitive EPI sequence was used for all functional scans (matrix $64 \times 64,24$ slices, slice thickness 4 or $4.5 \mathrm{~mm}$, FoV 192 or $224 \mathrm{~mm}^{2}$ (both depending on the subject's head size), resulting voxel size $3 \times 3 \times 4$ or $3.5 \times 3.5 \times 4.5 \mathrm{~mm}^{3}$, TE/TRslice $30 / 63$ $\mathrm{ms}$ (block runs) and 30/62.5 (event-related runs), FA $90^{\circ}$ ). In the passive block runs, the sequence repeat time (TR) was $5200 \mathrm{~ms}$, sequence scanning time was $1512 \mathrm{~ms}$, delay within a TR was $3688 \mathrm{~ms}$. During experimental blocks, 5 stimuli were presented in this delay (see figure 4.1B). In the event-related runs, the sequence repeat time (TR) was $2000 \mathrm{~ms}$, sequence scanning time was $1500 \mathrm{~ms}$, delay within a TR was $500 \mathrm{~ms}$. During experimental trials, one stimulus was presented in this delay (see figure $4.1 \mathrm{C}$, Study 1). A high-resolution structural scan (voxel size: $1 \times 1 \times 1 \mathrm{~mm}^{3}$ ) was collected for each subject using a T1-weighted 3D MP-RAGE sequence (Magnetization-Prepared Rapid Acquisition Gradient Echo, TR $=2.3 \mathrm{~s}, \mathrm{TE}=3.93 \mathrm{~ms}$, 192 sagittal slices).

Analysis of $f M R I$ time-series: Functional and anatomical images were analyzed using BrainVoyager QX (Brain Innovation, Maastricht, The Netherlands). The following preprocessing steps were performed: slice scan time correction (using sinc interpolation), linear trend removal, temporal high pass filtering to remove lowfrequency nonlinear drifts of 3 or less cycles per time course, and 3D motion correction to detect and correct for small head movements by spatial alignment of all volumes to the first volume by rigid body transformations. Functional slices were co-registered to the anatomical volume using position parameters from the scanner and manual adjustments to obtain optimal fit, and transformed into Talairach space.

The fMRI time-series were analyzed using fixed-effects multiple linear regression models (GLM) in which all experimental conditions were modeled as predictors. Predictor time-courses were adjusted for the hemodynamic response delay by convolution with a hemodynamic response function (Boynton et al., 1996). In the passive experiments (block and event-related), the GLM contained four predictors: unimodal visual, unimodal auditory, bimodal congruent and bimodal incongruent. In the active experiment, there were only two conditions: bimodal congruent and bimodal incongruent. Only the correctly answered trials were included in the predictors 
corresponding to the experimental conditions, incorrectly answered trials were modeled as a separate predictor. This resulted in three predictors: bimodal congruent, bimodal incongruent and incorrect trials.

To explore the effects of presentation mode (block vs. event-related) and top-down task-related factors (passive vs. active), we used the congruency effect (congruent vs. incongruent), which robustly revealed regions in the posterior auditory association cortex (planum temporale, PT) in the two previous AMRI studies (Van Atteveldt et al., submitted [chapter 3]; Van Atteveldt et al., 2004 [chapter 2]). Regions in the PT displaying a congruency effect in the different experiments of the current study were saved as ROIs, to enable ROI-based analyses on the time-series from the two experiments not used for defining the ROI (e.g.: ROIs defined by the passive block experiment allow ROI-based analysis in the passive and active event-related experiments). Furthermore, time-courses of all conditions were examined in these ROIs for all three experiments, to directly compare the response pattern in the PT in the different experiments.

\section{A behavioral experiment}

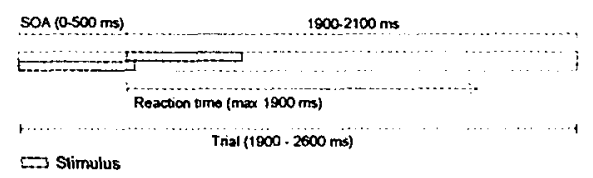

B fMRI experiments - Block runs

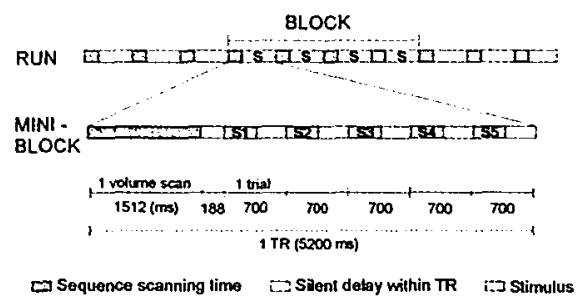

C FMRI experiments - Event-related runs
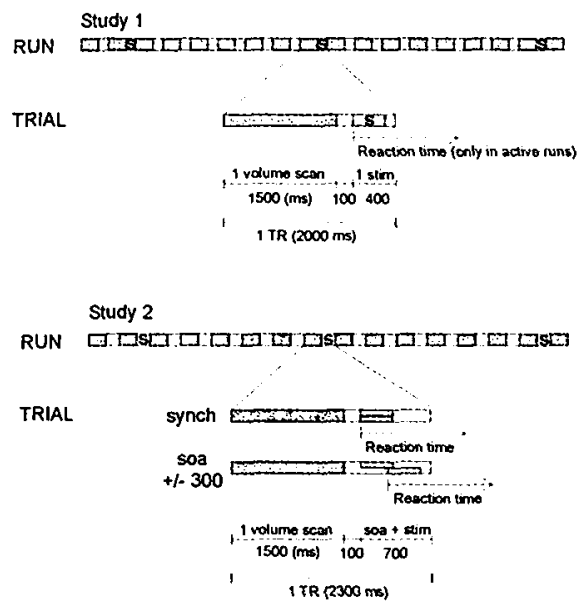

Figure 4.1. Schematic description of the different experimental designs used in studies 1 and 2.

(A) Trials in the behavioral experiment. At the beginning of each trial, a visual and an auditory stimulus were presented at different SOAs (0-500 ms in both directions). Reaction times were measured from the onset of the first stimulus.

(B) Block design used in fMRI experiments in studies 1 and 2. Experimental blocks were composed of 4 mini-blocks. Each mini-block started with the acquisition of one volume scan followed by 5 stimuli (SI-5) presented in the silent delay period.

(C) Event-related design used in fMRI experiments in studies 1 (upper figure) and 2 (lower figure). Study I: experimental trials (every $7^{\text {th }}, 8^{\text {th }}$ or $9^{\text {th }}$ TR) started with the acquisition of one volume scan followed by a stimulus (S) presented in the silent delay period. Reaction times (only in the active runs) were measured from the onset of the stimulus pair. Study 2: the scanning sequence was identical to study l except for a longer silent delay period. The stimuli were presented at different SOAs: -300 (visual first), 0 (synch = synchronous) or 300 (auditory first) $\mathrm{ms}$. Reaction times were measured from the onset of the first stimulus. SOA, stimulus onset asynchrony; TR, sequence repeat time (sequence scanning time + silent delay); Stim, stimulus. 


\section{Study 2}

\section{Behavioral experiment}

Participants: Ten healthy native Dutch subjects ( 2 males, 1 left-handed, mean age 27, range 23-33) participated in the behavioral experiment. All subjects were graduate students, had normal vision and hearing capacity, and no (history of) reading or other language problems.

Task procedure: Subjects were tested individually in a sound-attenuating room, in front of a computer monitor, on which the visual stimuli (single letters) were presented. Speech sounds were presented binaurally through loudspeakers. Pairs of letters and speech sounds were presented sequentially (see experimental trials). After each stimulus pair, subjects had to give a yes/no response to the question: "did you see and hear the same letter?" by pressing one of two buttons as fast and accurate as possible. The correct button (left/right) for "yes" and "no" was counterbalanced over the subjects.

Experimental trials: In each trial, one letter and one speech sound were presented (figure 4.1A). Congruency (congruent, incongruent) and stimulus onset asynchrony (SOA) were randomly distributed over all trials. Eleven SOAs were sampled: -500 , $300,-150,-100,-50,0,50,100,150,300$ and $500 \mathrm{~ms}$. The visual stimulus preceded in trials with a negative SOA, the auditory stimulus preceded in trials with a positive SOA. In total, there were 22 conditions (2 levels of congruency x 11 levels of SOA), 48 trials were presented per condition, resulting in 1056 trials in total. Stimulus presentation time was $350 \mathrm{~ms}$. Reaction times (RT) were recorded from the onset of the second stimulus for $1900 \mathrm{~ms}$. The time between the second stimulus of a trial and the start of the next trial was 1900,2000 or $2100 \mathrm{~ms}$, resulting in a trial duration in the range $1900-2600 \mathrm{~ms}$ (see figure 4.1A). Both accuracy (\% correct responses) and RT (only correct responses) measures were analyzed using a $2 * 11$ (congruency*SOA) within-subject ANOVA.

\section{fMRI experiment}

Participants: Sixteen healthy native Dutch subjects participated in the second fMRI experiment. Three subjects were excluded from data analyses due to excessive head motion during the functional scans. The remaining 13 subjects ( 4 male, mean age 23 , range 18-34) were all university students enrolled in an (under) graduate study program. We selected subjects based on a questionnaire including questions concerning (present or history of) reading or other language problems. All were right-handed, had normal or corrected-to-normal vision, and normal hearing capacity. Subjects gave informed written consent and were paid for their participation.

Experimental procedure: In each subject, 2 different experiments were conducted: a passive block experiment identical to study 1 (passive experiment), and an active event-related experiment similar to study 1 but with different temporal offsets (SOAs) between the visual and auditory stimulus (active experiment). The passive experiment was always conducted first. Three SOAs were sampled in the active experiment: -300 (visual first, VA), 0 (synchronous, SYN) and +300 (auditory first, AV) ms (see figure 4.1C, study 2). This resulted in 6 conditions in the active experiment $(2 * 3$, congruency*SOA), 6 trials of each condition were presented in each run. Similar to the behavioral experiment and fMRI experiment 1 , the subjects were instructed to respond 
as fast and accurately as possible to the question: "did you see and hear the same letter?" after each stimulus pair. Reaction times were recorded from the onset of the second stimulus. The correct button (middle or index finger of the right hand) for "yes" and "no" was counterbalanced over the subjects.

Scanning procedure: Imaging was performed on a 3 Tesla head scanner (Magnetom Allegra, Siemens Medical Systems, Erlangen, Germany) located at the M-BIC in Maastricht, The Netherlands. In each subject, 3 or 4 passive block runs of 70 volumes were acquired, followed by 3-5 active event-related runs of 262 volumes, using a BOLD-sensitive EPI sequence (scanning parameters: see study 1). In the passive runs, the scanning sequence was identical to that used in study 1 (see figure 4.1B). In the active runs, the scanning sequence was also identical to that used in study 1 , except that the sequence repeat time was changed from $2000 \mathrm{~ms}$ to $2300 \mathrm{~ms}$, creating a longer delay $(800 \mathrm{~ms})$ which was needed for the SOA $(300 \mathrm{~ms})$ between the visual and auditory stimulus (figure 4.1C, Study 2). A high-resolution structural scan (voxel size: $1 \times 1 \times 1 \mathrm{~mm}^{3}$ ) was collected for each subject using a T1-weighted 3D MP-RAGE sequence (Magnetization-Prepared Rapid Acquisition Gradient Echo, TR $=2.3 \mathrm{~s}, \mathrm{TE}=$ $3.93 \mathrm{~ms}, 192$ sagittal slices).

Analysis of fMRI time-series: Functional and anatomical images were analyzed using BrainVoyager QX (Brain Innovation, Maastricht, The Netherlands). The preprocessing procedure was identical to the fMRI experiment in study 1. The fMRI time-series of both experiments were analyzed using random-effects multiple linear regression models (GLM) in which all experimental conditions were modeled as separate predictors. Only correctly answered trials were included in these experimental predictors in the active experiment, incorrectly answered trials were modeled as a separate predictor. Predictor time-courses were adjusted for the hemodynamic response delay by convolution with a two gamma hemodynamic response function (in which both the BOLD response and the undershoot are modeled by a gamma function). In the passive experiment, the GLM contained four predictors: unimodal visual, unimodal auditory, bimodal congruent and bimodal incongruent. In the active experiment, the GLM contained seven predictors: bimodal congruent at the three SOAs (Con_VA, Con_Syn, Con_AV) and bimodal incongruent at the three SOAs (Inc_VA, Inc_Syn, Inc $\bar{A}$ ), and $\bar{a}$ predictor for the incorrect trials. To search for interactions between SOA and congruency, the active runs were also analyzed using a $2 * 3$ (congruency*SOA) factorial model (Van Atteveldt et al., submitted [chapter 3]), in which predictors were defined for the main effects of congruency and SOA, the interaction between congruency*SOA, and for the incorrectly answered trials.

We used two different analytical strategies to infer experimental effects from the fMRI time-series in study 2. In the first strategy, we performed voxel-wise (whole-brain) GLM analysis on the passive runs, and subsequently performed ROI-based analyses on the time-series from the active experiment. These analyses were performed both on a group level and on the level of individual subjects. This first analytical strategy allowed us to directly compare the fMRI responses during active integration at different SOAs in regions found to be involved in integration during the passive experiment. In the second, more explorative analytical strategy, we performed wholebrain analyses directly on the time-series from the active experiment. This second analytical strategy allowed us to explore the effect of congruency during active integration in regions not identified during passive integration. 
First analytical strategy: Voxel-wise multi-subject GLM analyses were performed on the passive runs to replicate our previous findings (Van Atteveldt et al., 2004 [chapter $2]$ ). Based on the results of this previous study, we searched for regions showing an effect of congruency (congruent $>$ incongruent) in the auditory association cortex. We saved these regions as ROIs, and performed ROI-based analyses on the time-series from the active experiment. On the group level, one ROI was selected in each hemisphere (at $q(F D R)<0.05$ ). In individual subjects, ROIs were selected using the congruency contrast in single-subject voxel-wise GLM analyses. In each subject, we selected an ROI in each hemisphere located on the planum temporale (based on anatomical information from Duvernoy (1999)) in which the fMRI response showed the following pattern: Congruent $>$ Auditory $>$ Incongruent $>$ Visual. Thresholds were adjusted in each subject to yield ROIs with approximately the same cluster size. To examine the experimental effects in the active runs in these individual ROIs, we extracted the maximum \% signal change for congruent and incongruent trials from the averaged time-course values.

Second analytical strategy: We performed voxel-wise (whole brain) GLM analyses directly on the time-series from the active experiment, to further explore the differences between active and passive integration, and to examine the effect of temporal relation on active letter-sound integration. For these purposes, we searched for voxels showing an effect of congruency (congruent vs. incongruent) or an interaction between $\mathrm{SOA}^{*}$ congruency. Interactions between SOA and congruency were evaluated with the $2 * 3$ factorial GLM, congruency effects with the single factor GLM. To estimate effects of congruency, the three congruent (Con_VA, Con_Syn, Con_AV) predictors were contrasted with the three incongruent (Inc_VA, Inc_Syn, Inc_AV) predictors. Clusters showing experimental effects were shown on the inflated cortical surfaces of the MNI template brain, and in sagittal or transversal slices of the $3 \mathrm{D}$ anatomical image of the MNI template brain. Clusters showing experimental effects were assigned to corresponding anatomical structures using Duvernoy (1999).

\section{Results}

\section{Study 1}

Behavioral results: In the active event-related experiment, no significant differences in reaction time were found (congruent: $836.8 \mathrm{~ms}$, incongruent: $864.8 \mathrm{~ms}$, paired samples $\mathrm{t}$-test: $\mathrm{t}=1.1, \mathrm{p}=0.32$ ). Average accuracy percentages were all above $90 \%$, and also not significantly different (congruent: $90.7 \%$, incongruent $91.6 \%, t=1.03, p=0.35$ ). Because only the fMRI responses to correctly answered trials were analyzed, the high accuracy levels indicate that only very few trials had to be discarded in the fMRI analysis.

fMRI results: figure 4.2 shows the results of the congruency contrast (congruent vs. incongruent) in the different experiments. For both passive experiments, similar regions in auditory association cortex were revealed that showed a stronger response to congruent than to incongruent stimulation (shown in figure 4.2A). In contrast, no regions in the auditory cortex showed an effect of congruency in the active experiment. In figure 4.2B, averaged BOLD response time courses are shown for ROIs selected by the passive block experiment (left column, representative for yellow ROIs in figure $4.2 \mathrm{~A}$ ) and the passive event-related experiment (right column, representative for red 
ROIs in figure 4.2A). Although the shape and amplitude of the signal changes are different between the different experiments (as expected, see below), the response patterns are remarkably similar for the two passive experiments (Congruent > Auditory $>$ Incongruent $>$ Visual). In contrast, the time courses for congruent and incongruent stimuli are identical in the active experiment.

These observations were confirmed by ROI-based analyses performed on the two experiments not used for defining the ROI (table 4.1). In the ROI selected by the passive block experiment $(-38,-27,12)$, the congruency effect was significant in the passive event-related runs $(t=2.19, p=0.028)$, but not in the active event-related runs $(t=-0.98, p=0.33)$. In the ROI selected by the passive event-related experiment $(-45$, $19,7)$, the congruency effect was significant in the passive block experiment $(t=3.40$, $p=0.0007)$, but again, not in the active event-related experiment $(t=-0.65, p=0.52)$.

The different shape and amplitude of the signal changes are as expected from the stimulus duration differences (see "introduction"). The finding of similar response patterns indicates that the event-related fMRI design is suitable to find the desirable experimental effects, in spite of the different stimulation duration and relative amount of scanner noise.

Table 4.1. ROI-based analysis study 1

\begin{tabular}{|c|c|c|c|c|}
\hline \multirow{2}{*}{$\begin{array}{l}\text { Experiment used for ROI } \\
\text { selection by [congruent }> \\
\text { incongruent] }\end{array}$} & \multirow{2}{*}{$\begin{array}{c}\text { ROI } \\
(x, y, z)\end{array}$} & \multicolumn{3}{|c|}{ ROI-based analysis [Congruent $>$ Incongruent] } \\
\hline & & Passive block & $\begin{array}{l}\text { Passive event- } \\
\text { related }\end{array}$ & $\begin{array}{c}\text { Active } \\
\text { event-related }\end{array}$ \\
\hline Passive block & $-38,-27,12$ & - & $p=0.028$ & $\mathrm{p}=0.33$ \\
\hline Passive event-related & $-45,-19,7$ & $\mathrm{p}=0.0007$ & - & $\mathrm{p}=0.52$ \\
\hline
\end{tabular}




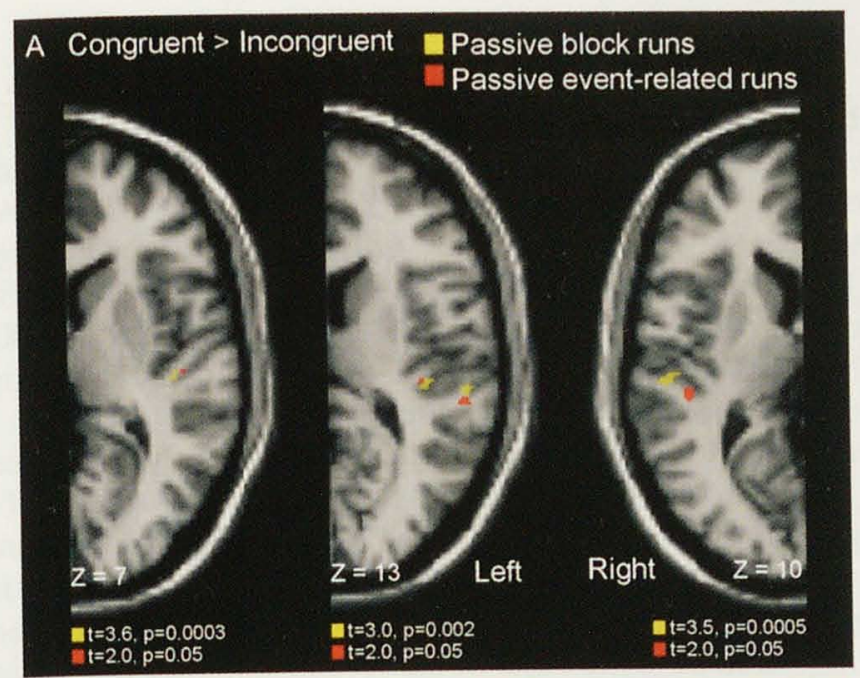

B ROIs selected by passive block runs
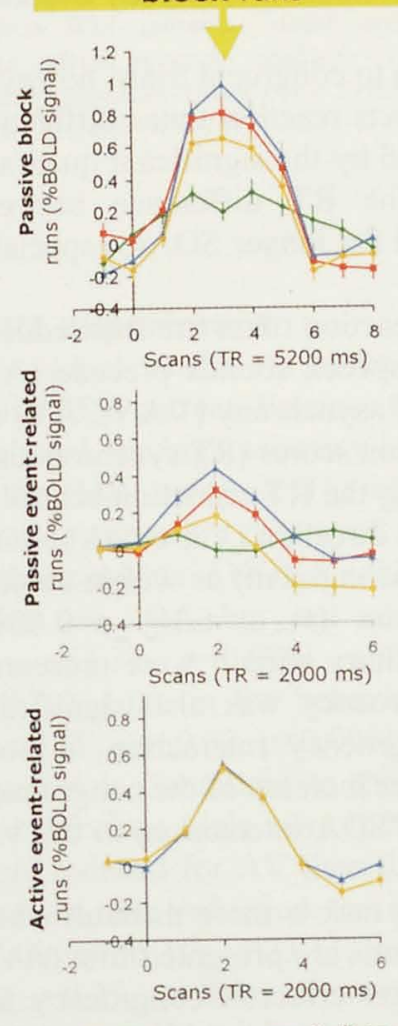

- Visual - Auditory
ROIs selected by passive event-related runs
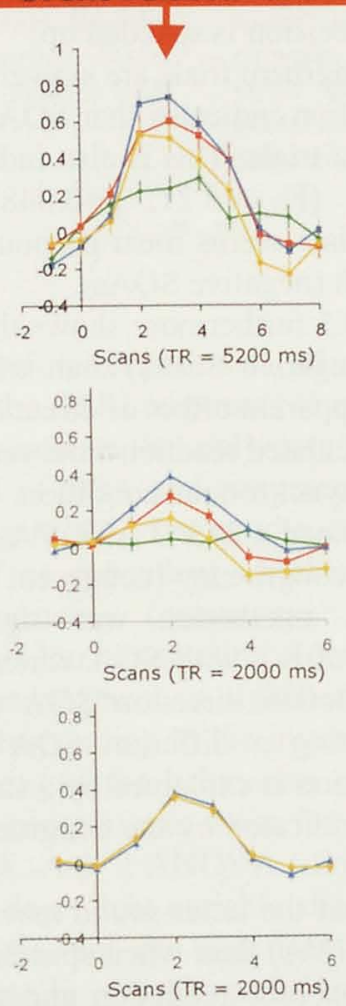

- Congruent - Incongruent
Figure 4.2. Group Results of the congruency contrast in study 1 .

(A) ROIs in the planum temporale shown for both passive experiments (yellow: block, red: event-related), projected in transversal slices of the MNI template brain. No voxels were found in the planum temporale in the active experiment.

(B) Averaged BOLD response time courses for the ROIs shown in A, selected by the passive block left column) or passive eventrelated (right column) experiments. Averaged timecourses are shown for the passive block (top row). passive event-related (middle row) and active event-related (lower row) experiments. TR, sequence repeat time (scan acquisition + silent delay). 


\section{Study 2}

\section{Behavioral experiment}

The $2 * 11$ (congruency*SOA) within-subjects ANOVA with accuracy as dependent variable revealed no significant effects (congruency: $F_{(1,9)}=0.13, p=0.7$, SOA: $\mathrm{F}_{(10.90)}=1.06, \mathrm{p}=0.4$, congruency*SOA: $\mathrm{F}_{(10,90)}=0.88, \mathrm{p}=0.5$ ), all average accuracy levels were above $95 \%$. Using RT as dependent variable, significant experimental effects were observed (congruency: $F_{(1,9)}=19.2, p=0.002$, SOA: $F_{(10,90)}=64.3, p<0.000$, congruency*SOA: $\left.F_{(10,90)}=2.38, p=0.015\right)$. The effects of SOA and congruency on letter-sound matching reaction times are displayed in figure 4.3.

Figure 4.3 shows that reaction times were longest for the synchronously presented letter-sound pairs (SOA $=0 \mathrm{~ms}$ ). In both directions (visual first and auditory first), RTs were reduced with increasing SOA, as shown by the inverted U-shaped curves in figure 4.3 (significant quadratic trend SOA: $F_{(1,9)}=420.75, p<0.000$ ). This reduction is stronger when sounds are presented first (positive SOAs), which will be discussed below. Reaction times were measured from the onset of the second stimulus, indicating that the decision regarding congruency is faster when information about one of the stimuli is already available. Moreover, the longer this information is already available (longer SOA), the more the decision is speeded up.

On average, reactions to incongruent trials are slower than to congruent trials, however, the congruency*SOA interaction indicates that SOA affects reaction times differently for congruent and incongruent trials. This is also indicated by the significant quadratic trend for congruency*SOA $\left(\mathrm{F}_{(1,9)}=5.21, \mathrm{p}=0.048\right)$. The RT difference between incongruent and congruent trials seems most pronounced for longer SOAs, especially when letters are presented first (negative SOAs).

As mentioned above, figure 4.3 furthermore shows that reaction times are less reduced when letters precede (VA, negative SOAs) than when speech sounds precede (AV, positive SOAs). To test this apparent effect of direction of asynchrony (VA vs. AV) on letter-sound matching, we calculated reaction-time-reduction scores (RT synchronous $\mathrm{RT}$ asynchronous) for each asynchronous condition. Using the RT-reduction-scores as dependent variable, we performed a $2 * 5 * 2$ ANOVA with direction (VA or AV), SOA $(50,100,150,300,500)$ and congruency (congruent or incongruent) as within-subject factors. The direction*SOA interaction was significant $\left(F_{(4.36)}=6.18, p=0.001\right)$, indicating a larger reduction of RT with SOA when auditory stimuli were presented first (AV). The 3-way interaction direction*SOA*congruency was also significant $\left(F_{(4,36)}=3.03, p=0.03\right)$, indicating a different SOA*congruency interaction in both directions. This 3-way interaction is explained by a stronger increase of the congruency effect with SOA (as already indicated by the congruency*SOA interaction) in the VA direction.

These observations suggest that the letter-sound matching task is more difficult when letters precede speech sounds (VA) than when speech sounds are presented first (AV), as pronounced by less reaction time reduction and a larger effect of congruency for VA. Based on the results of the behavioral experiment, we selected $-300 \mathrm{~ms}$ and 300 $\mathrm{ms}$ as SOAs for in the fMRI experiment, because the effects of congruency and direction of asynchrony are clearly present at these SOAs. 


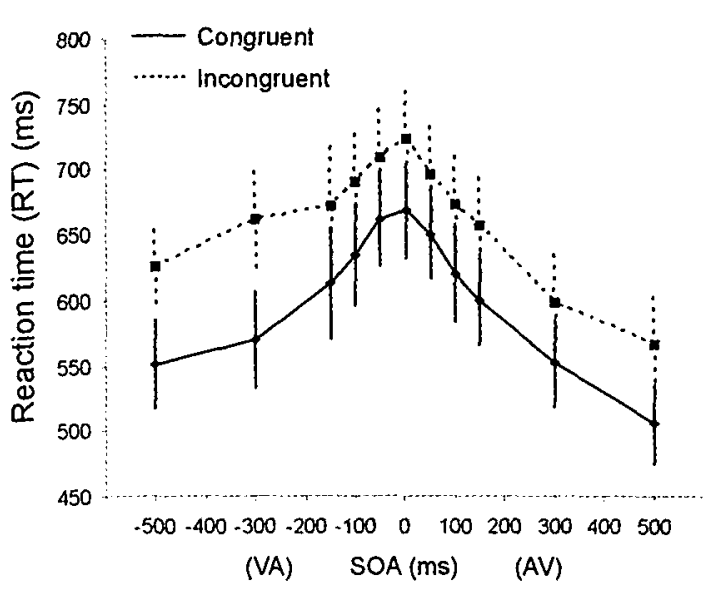

Figure 4.3. Reaction Time Results from the Behavioral Experiment.

Average reaction times for congruent (solid line) and incongruent (dashed line) letter-sound pairs are plotted against SOA (stimulus onset asynchrony). Negative SOAs: visual precedes auditory (VA), positive SOAs: audirory precedes visual (AV). Error bars indicate $S E M$ across subjects $(n=10)$.

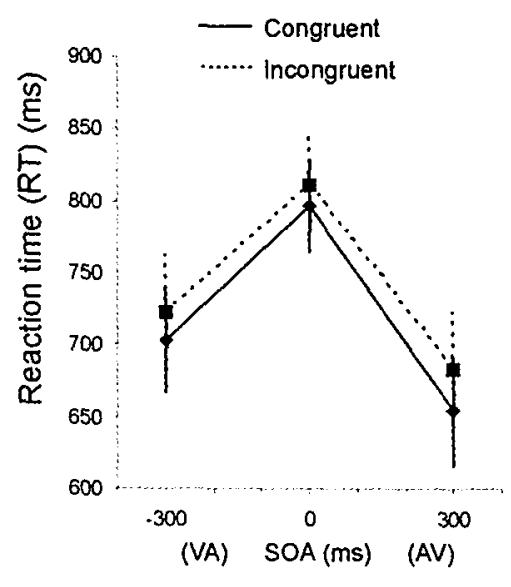

Figure 4.4. Reaction Time Results from the Active fMRI Experiment in Study 2.

Average reaction times for congruent (solid line) and incongruent (dashed line) lettersound pairs are plotted against SOA (stimulus onset asynchrony). Negative SOAs: visual precedes auditory (VA), positive SOAs: auditory precedes visual (AV). Error bars indicate SEM across subjects $(n=13)$.

\section{fMRI experiment}

Behavioral results: As in the behavioral experiment, all average accuracy levels were above $95 \%$ correct. Because only the fMRI responses to correctly answered trials were analyzed, the high accuracy levels indicate that only very few trials had to be discarded in the fMRI analysis. The effects of SOA and congruency on letter-sound matching reaction times are displayed in figure 4.4 . The results of the $2 * 3$ (congruency*SOA) within-subjects ANOVA with RT as dependent variable revealed a significant main effect of SOA but not of congruency (congruency: $F_{(1,12)}=1.51, p=0.24$, SOA: $F_{(2,24)}=52.32, p<0.000$ ), and also no interaction (congruency*SOA: $F_{(2,24)}=0.36$, $\mathrm{p}=0.70$ ). Like in the behavioral experiment, a significant linear trend of SOA was found $\left(F_{1,12}=60.82, p<0.000\right)$, corresponding to the inverted $U$-shaped curve shown in figure 4.4. Consistent to the results of the behavioral experiment, reaction times to asynchronous trials were reduced compared to those for synchronous trials, and again more reduced for AV than for VA. A $2 * 2$ ANOVA (direction*congruency) using the reaction-time-reduction-scores as dependent variable showed a significant main effect of direction $\left(\mathrm{F}_{1,12}=23.11, \mathrm{p}<0.000\right)$, but no significant main effect of congruency $\left(F_{1,12}=0.29, p=0.6\right)$ or interaction $\left.F_{1,12}=0.49, p=0.5\right)$. So, like in the behavioral experiment, VA trials seem more difficult than AV trials during the fMRI experiment.

fMRI results: Analyses based on passive experiment

Multi-subject analysis: In the first analytical strategy, we defined ROIs based on the congruency contrast in the passive block runs. Figure 4.5A shows the results of the first 
analysis on the group level. One ROI was selected for the congruency contrast in each hemisphere (see table 4.2, group ROIs). The averaged time-courses of the BOLD response in the passive experiment showed an auditory specific response (auditory unimodal $>$ visual unimodal), as well as a modulation of the auditory response during bimodal stimulation (congruent $>$ auditory and incongruent $<$ auditory). This pattern of enhancement and suppression of the response to speech sounds is a replication of our previous results (Van Atteveldt et al., 2004 [chapter 2]). The averaged time-courses of the BOLD response in the active experiment in the same ROIs showed identical responses to congruent and incongruent letter-sound pairs at all SOAs. The results of ROI-based statistics on the time-series of the active experiment confirmed this observation: the difference between congruent and incongruent was not significant at any SOA (Left, VA: $t=-0.997, p=0.32$; $S Y N: t=-1.696, p=0.09, A V: t=-1.320, p$ $=0.19$; Right, VA: $\mathrm{t}=-1.475, \mathrm{p}=0.14, \mathrm{SYN}: \mathrm{t}=-0.448, \mathrm{p}=0.65, \mathrm{AV}: \mathrm{t}=-1.455, \mathrm{p}=$ $0.15)$.

Single-subject analysis: Next, we used single-subject GLM analyses to define ROIs based on the congruency contrast in the passive experiment in individual subjects. The details of the resulting ROIs are given in table 4.2. In the left hemisphere, an ROI located on the planum temporale showing the correct response pattern was found in all 13 subjects, and in 11/13 subjects in the right hemisphere. The maximum $\%$ signal change in the individual ROIs (averaged across subjects) plotted in figure $4.5 \mathrm{~B}$ show no effects of congruency or SOA. This observation was confirmed by the results of the ANOVA with maximum $\%$ signal change as dependent variable (Congruency: $\mathrm{F}_{1,12}=0.36, \mathrm{p}=0.58$ (left), $\mathrm{F}_{1,10}=0.14, \mathrm{p}=0.72$ (right); $\mathrm{SOA}: \mathrm{F}_{2,24}=0.81, \mathrm{p}=0.46$ (left), $\mathrm{F}_{2,20}=0.29, \mathrm{p}=0.75$ (right); Congruency*SOA: $\mathrm{F}_{2,24}=0.006, \mathrm{p}=0.99$ (left), $\mathrm{F}_{2,20}=0.01$, $\mathrm{p}=0.99$ (right)). For comparison, the maximum \% signal change for the passive experiment in the same individual ROIs are also shown in figure $4.5 \mathrm{~B}$.

Table 4.2. ROIs in planum temporale defined by passive runs [congruent $>$ incongruent]

\begin{tabular}{|c|c|c|c|c|c|c|c|c|c|c|}
\hline \multirow[b]{2}{*}{ Subjects } & \multicolumn{3}{|c|}{ Left hemisphere } & \multirow[b]{2}{*}{$\begin{array}{c}\text { Cluster } \\
\text { size } \\
\end{array}$} & \multirow[b]{2}{*}{$t$} & \multicolumn{3}{|c|}{ Right hemisphere } & \multirow[b]{2}{*}{$\begin{array}{c}\text { Cluster } \\
\text { size }\end{array}$} & \multirow[b]{2}{*}{$\mathrm{t}$} \\
\hline & $\mathrm{x}$ & $y$ & z & & & $x$ & $y$ & $\mathbf{z}$ & & \\
\hline \multicolumn{11}{|l|}{ Group: } \\
\hline \multicolumn{11}{|c|}{ Individual subjects: } \\
\hline$\# 1$ & -37 & -27 & 16 & 39 & 3.0 & 49 & -25 & 7 & 37 & 2.6 \\
\hline$\# 2$ & -39 & -21 & 11 & 43 & 3.4 & 51 & -21 & 14 & 34 & 3.7 \\
\hline \#3 & -48 & -37 & 16 & 26 & 3.2 & 54 & -27 & 12 & 31 & 3.4 \\
\hline \#4 & -48 & -28 & 20 & 28 & 3.2 & 53 & -16 & 11 & 38 & 3.6 \\
\hline \#5 & -53 & -20 & 7 & 27 & 3.2 & - & - & - & - & - \\
\hline \#6 & -58 & -19 & 17 & 29 & 4.2 & 40 & -19 & 9 & 48 & 3.0 \\
\hline$\# 7$ & -53 & -24 & 6 & 23 & 2.3 & 46 & -13 & 6 & 29 & 2.3 \\
\hline$\# 8$ & -63 & -23 & 2 & 33 & 4.8 & 47 & -21 & 12 & 35 & 2.5 \\
\hline$\# 9$ & -46 & -18 & 4 & 26 & 2.8 & 51 & -25 & 11 & 21 & 2.5 \\
\hline$\# 10$ & -34 & -28 & 14 & 41 & 3.0 & 42 & -27 & 8 & 42 & 2.8 \\
\hline$\# 11$ & -48 & -34 & 18 & 23 & 3.6 & 51 & -14 & 16 & 36 & 3.3 \\
\hline$\# 12$ & -48 & -25 & 4 & 23 & 2.8 & - & - & - & - & - \\
\hline$\# 13$ & -59 & -18 & 5 & 33 & 2.9 & 63 & -19 & 6 & 43 & 4.2 \\
\hline Average & -48.8 & -24.8 & 10.8 & 30.3 & 3.3 & 49.7 & -20.6 & 10.2 & 35.8 & 3.2 \\
\hline (St dev) & 8.6 & 6.0 & 6.3 & 7 & 0.7 & 6.2 & 5.0 & 3.3 & 7.3 & 0.6 \\
\hline
\end{tabular}


A
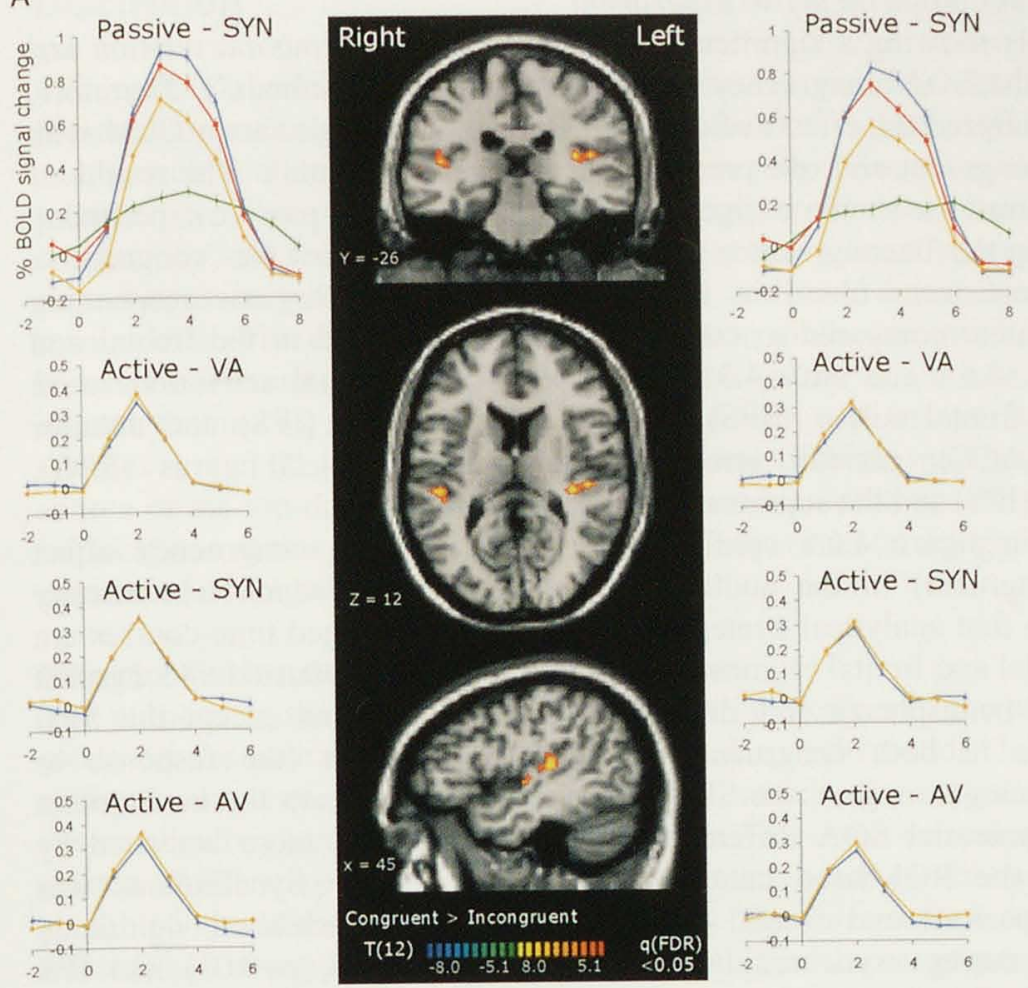

- Visual - Auditory

- Congruent - Incongruent
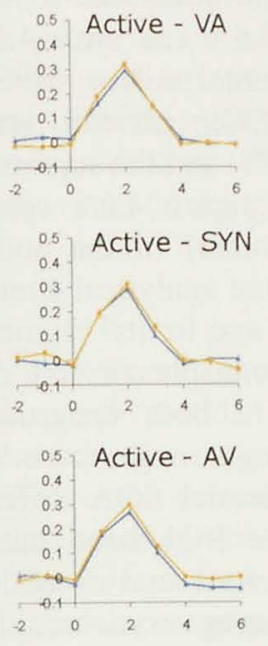

B Left ROIs

Right ROIs
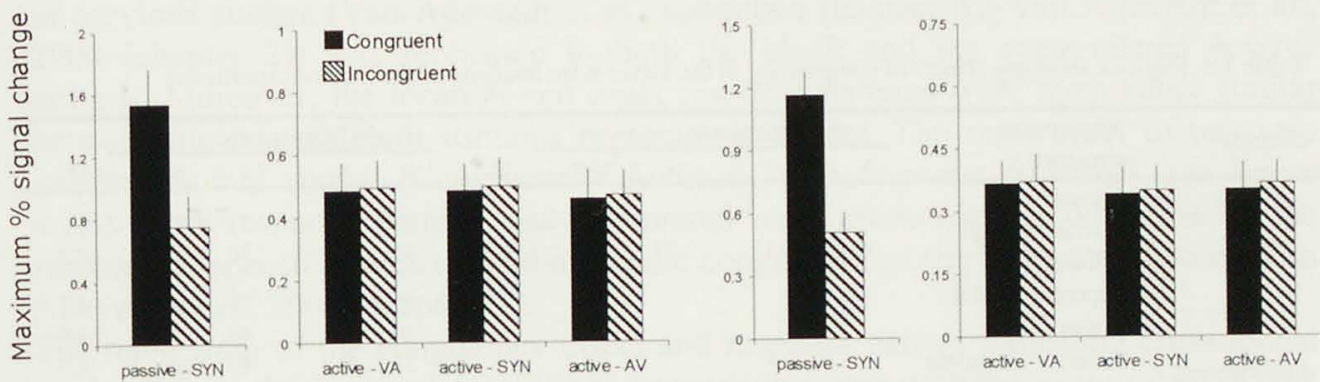

Figure 4.5. fMRI results from study 2: analyses based on the passive experiment.

(A) Group results. Middle panel: statistical map of the congruency contrast, projected on the MNI template brain. Graphs: averaged BOLD response time-courses for the superior temporal regions in left (graphs on the right) and right (graphs on the lefi) hemisphere. Top row: time courses of the passive experiment (Passive SYN), rows below: time courses of the active experiment, plotted separately for the different SOAs (Active $V A$, Active $-S Y N$, Active - AV). SOA = stimulus onset asynchrony, $V A=$ visual precedes auditory $(S O A=-300$ $m s), S Y N=$ synchronous $(S O A=0 \mathrm{~ms}), A V=$ auditory precedes visual $(S O A=300 \mathrm{~ms})$.

(B) Single-subject results. Maximum \% signal change in the individual ROIs averaged across subjects, plotted separately for the passive experiment (Passive - SYN) and the active experiments at different SOAs (Active $V A$, Active - SYN, Active - AV). Error bars indicate SEM across subjects. 
fMRI results: Analyses based on active experiment

We found no voxels showing a significant interaction between temporal relation and congruency using the SOA*congruency factorial model (see "Methods"). Therefore, we subsequently analyzed the effects of congruency using the single factor GLM with one predictor for congruent and one predictor for incongruent stimuli. The results of the congruency contrast are shown in figure 4.6. As opposed to the passive experiment, only voxels showing the "incongruent > congruent" contrast, but not the "congruent $>$ incongruent" contrast were found in the active experiment. Regions responding stronger to incongruent compared to congruent trials were found in the frontal and parietal cortex (fig. 4.6A and table 4.3). More specifically, frontal activations were found in superior frontal sulcus (SFS), inferior frontal sulcus (IFS) and anterior cingulate cortex (ACC), parietal activations in superior parietal gyrus (SPG), intraparietal sulcus (IPS) and the supramarginal gyrus (SMG).

The surface map in figure $4.6 \mathrm{~A}$ confirms the absence of the congruency effect (congruent $>$ incongruent) in the auditory association cortex, which was already demonstrated in the first analytical strategy. In figure 4.6B, averaged time-courses are shown for the parietal and frontal regions exhibiting a stronger response to incongruent trials, pooled over hemispheres and different SOAs. All regions except the SPG showed a response to both congruent and incongruent trials (the response to incongruent trials being stronger), the SPG responded exclusively to the incongruent trials. To explore potential SOA differences within the regions more activated by incongruent trials, the ROI-based contrast of Asynchronous > Synchronous was performed in the parietal and frontal regions. Two regions exhibited significant stronger responses during asynchronous trials: the IFS $(t=2.48, p=0.01)$ and IPS $(t=2.15, p=0.03)$. For these two regions, averaged time-courses for the different SOA conditions are shown separately for congruent and incongruent trials in figure $4.6 \mathrm{C}$.

Table 4.3. Regions showing effects of congruency in the voxel-wise analysis of the active experiment

\begin{tabular}{|c|c|c|c|c|c|c|c|c|c|c|c|}
\hline \multirow{2}{*}{ Contrast } & \multirow{2}{*}{$\begin{array}{l}\text { Anatomical } \\
\text { structure }\end{array}$} & \multicolumn{4}{|c|}{ Left hemisphere } & \multirow[b]{2}{*}{$t^{*}$} & \multicolumn{4}{|c|}{ Right hemisphere } & \multirow[b]{2}{*}{$\mathrm{t}^{*}$} \\
\hline & & $x$ & $y$ & $z$ & size & & $x$ & $y$ & $z$ & size & \\
\hline \multirow{9}{*}{$\begin{array}{l}\text { Incongruent } \\
> \\
\text { Congruent }\end{array}$} & $\begin{array}{l}\text { Fromial } \\
\text { Inferior frontal } \\
\text { sulcus (IFS) }\end{array}$ & -39 & 15 & 27 & 126 & 4.3 & 45 & 9 & 30 & 57 & 4.3 \\
\hline & Superior frontal & & & & & & 46 & 31 & 26 & 64 & 4.3 \\
\hline & sulcus (SFS) & & & & & & 30 & -7 & 49 & 76 & 4.6 \\
\hline & Anterior cingulate & & & & & & 31 & 0 & 49 & 46 & 4.3 \\
\hline & cortex $(\mathrm{ACC})$ & -5 & 14 & 46 & 32 & 4.3 & 8 & 14 & 48 & 231 & 4.5 \\
\hline & Parietal & & & & & & & & & & \\
\hline & $\begin{array}{l}\text { Superior parietal } \\
\text { gyrus (SPG) }\end{array}$ & -16 & -37 & 53 & 32 & 4.2 & 36 & -38 & 55 & 58 & 4.3 \\
\hline & $\begin{array}{l}\text { Intraparietal sulcus } \\
\text { (IPS) }\end{array}$ & -35 & -40 & 39 & 34 & 4.2 & 35 & -46 & 49 & 57 & 4.3 \\
\hline & $\begin{array}{l}\text { Supramarginal } \\
\text { gurus (SMG) }\end{array}$ & -46 & -38 & 32 & 75 & 4.6 & 51 & -41 & 25 & 119 & 4.4 \\
\hline
\end{tabular}

*Average t-value for all voxels in cluster 


\section{Discussion}

The principle aim of the present study was to examine the effects of stimulus presentation mode and top-down task-related factors on the neural integration of letters and speech sounds. Based on our previous results (Van Atteveldt et al., submitted [chapter 3]; Van Atteveldt et al., 2004 [chapter 2]), we explored the effects of congruency and temporal asynchrony in the auditory association cortex in a series of experiments in which stimulus presentation and task demands were systematically manipulated. The effect of stimulus presentation mode was investigated by comparing blocked and event-related experimental designs (study 1), the effect of top-down taskrelated factors by comparing passive and active versions of the same experiment (studies 1 and 2). After discussing the effects of both manipulations, the possible source of the top-down task effects will be considered, as are the specific and general implications of this systematic exploration of experimental design effects for studies of cross-modal integration.

\section{Effect of stimulus presentation mode}

The purpose of the first study was to explore the effect of the stimulus presentation mode on the neural integration of letters and speech sounds. In each subject, three different experiments were conducted in which the experimental design was systematically changed: a passive block, a passive event-related, and an active eventrelated fMRI experiment. In this study, letters and speech sounds were always presented synchronously in the bimodal trials. The intermediate design (passive eventrelated) had two goals: 1) testing the hypothesis that the congruency effect found in the previous studies can be explained by attention or arousal due to the blocked presentation, and 2) controlling for the changes in stimulus presentation mode unrelated to task factors (increased scanner noise and different stimulus duration).

As shown in figure 4.2 , the congruency effect in the auditory association cortex found in previous studies (Van Atteveldt et al., submitted [chapter 3]; Van Atteveldt et al., 2004 [chapter 2]) was replicated in both the block and the event-related passive designs. Moreover, the location and exact response patterns were remarkably similar between the two different stimulus presentation modes. The same order of response amplitudes was shown (Congruent $>$ Auditory $>$ Incongruent $>$ Visual, see figure 4.2B). This response pattern was interpreted as a cross-modal modulation of the response to speech sounds depending on the congruency of the letter-sound pairs (Van Atteveldt et al., 2004 [chapter 2]).

The replication of the congruency effect and response pattern indicating cross-modal modulation in the "unimodal" auditory association cortex with the event-related fMRI design has two important implications. First, it indicates that similar to blocks of lettersound pairs, single letter-sound pairs also elicit enhanced and suppressed responses (relative to speech sounds alone) in the auditory association cortex. This excludes the explanation of the congruency effect in terms of different attention levels in the block experiments, because each stimulus pair has to be integrated to determine the congruency in the event-related experiment. 


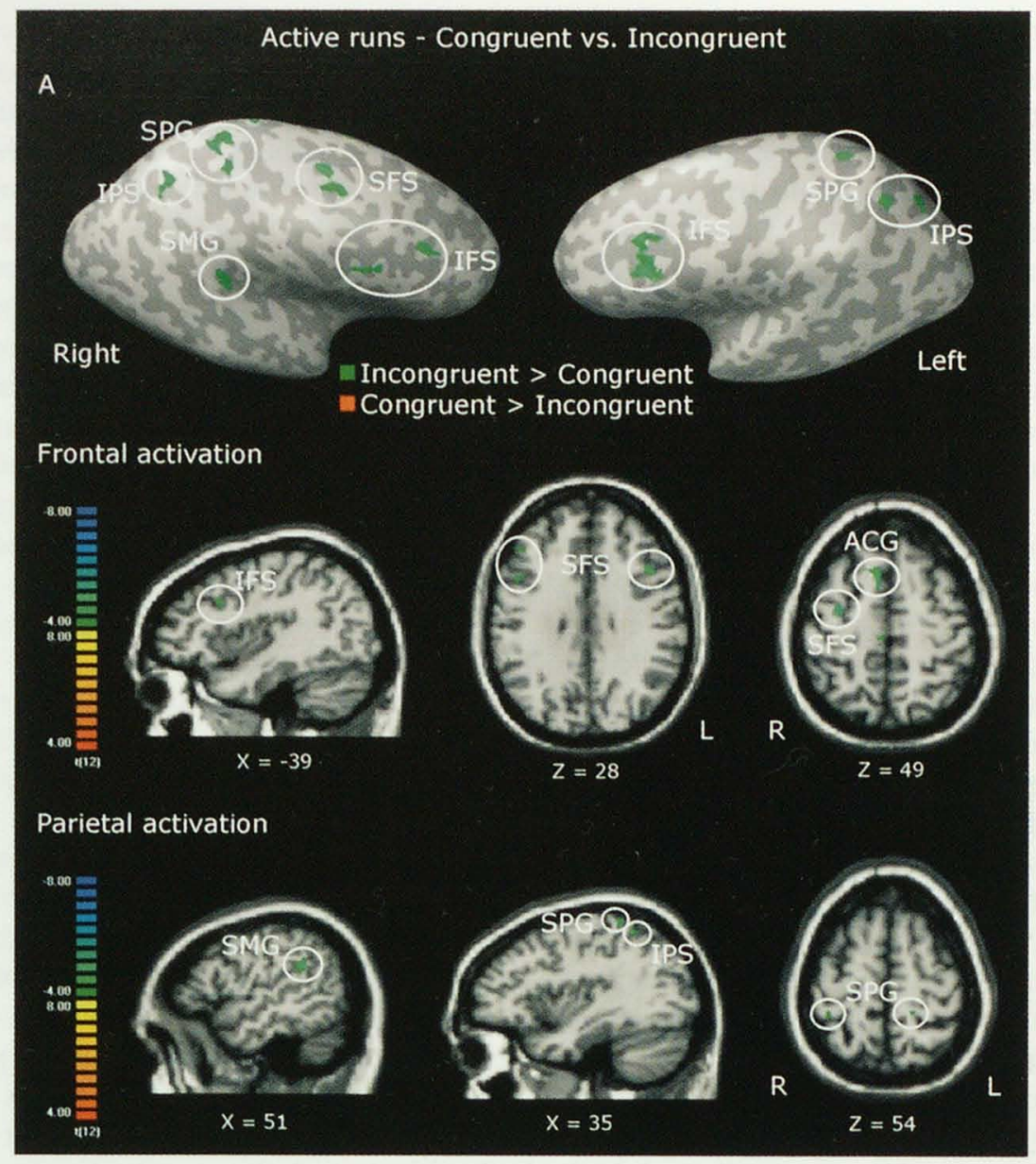

B
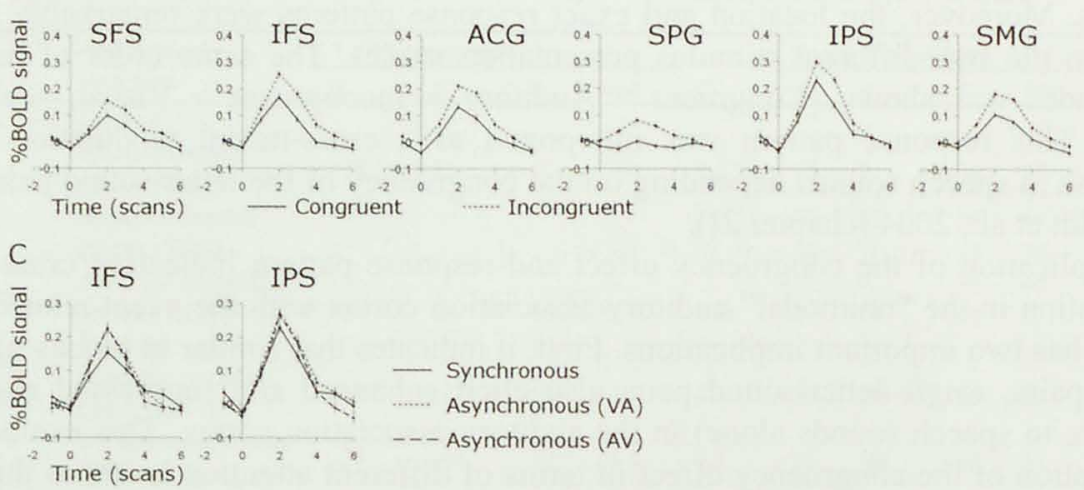

Figure 4.6. fMRI results from study 2: voxel-wise analyses of the active experiment. 
Figure 4.6 (Previous page). $f M R I$ results from study 2: voxel-wise analyses of the active experiment.

(A) Multi-subject statistical maps of the congruency contrast performed on the active runs, superimposed on the inflated cortical surfaces and $3 D$ slices of the MNI brain.

(B) Averaged time-courses of the BOLD response to congruent (solid lines) and incongruent (dashed lines) letter-sound pairs in the regions shown in A, pooled over hemispheres and SOAs.

(C) Averaged time-courses of the BOLD response to synchronous (solid lines) and asynchronous (VA, visual first: dotted lines; $A V$, auditory first: dashed lines) letter-sound pairs in the IFS and IPS, pooled over hemispheres and congruency.

SFS, superior frontal sulcus; IFS, inferior frontal sulcus; $A C G$, anterior cingulate gyrtus; SPG, superior parietal gyrus; IPS, intraparietal sulcus; SMG, supramarginal gyrus.

The second implication is crucial for the second part of the research question, the effect of top-down task-related factors on the neural integration of letters and speech sounds. The replication of the congruency effect with the event-related fMRI experiment excludes the possibility that different response patterns for active integration (using event-related $\mathrm{FMRI}$ ) and passive integration (using a block design) can be attributed to differences in experimental design related to stimulus duration and relative amount of scanner noise. The observation that the same response pattern (Congruent $>$ Auditory $>$ Incongruent $>$ Visual) was present in the event-related passive experiment, in spite of differences in scanner noise and stimulation duration, provides strong support for the suitability of the event-related fMRI experiment to detect differential responses to letters, speech sounds and their congruent and incongruent combinations. Therefore, the intermediate step (passive, event-related) was left out in study 2 , in which the effect of task-related factors was investigated in more detail.

\section{Top-down task effects}

In study 1 , the identical time-courses for congruent and incongruent stimuli in the active event-related runs (figure 4.2B) already indicated that the congruency effect was not present in the auditory association cortex during active matching of letters and speech sounds. The identical experimental design used in the passive and active eventrelated fMRI experiments in study 1 allows direct comparison of the averaged timecourses. Figure $4.2 \mathrm{~B}$ shows that responses in the auditory cortex to both congruent and incongruent stimuli are enhanced in the active experiment (lower row) when compared to the event-related passive experiment (middle row). It is also clear that the response to incongruent stimuli was enhanced the most, which resulted in the disappearance of the congruency effect. The general enhancement of the response in the auditory cortex may be caused by increased demands on attention during the active experiment, which has been reported before (e.g. Grady et al., 1997; Hall et al., 2000). As will be discussed later, the task instruction most likely changed the behavioral relevance of especially the incongruent stimuli, so the extra boosting of the response to incongruent stimuli in the auditory cortex indicates the task-specificity of the top-down influence. In study 2, top-down task effects on the neural mechanism of letter-speech sound integration were investigated in more detail, including the effect of temporal asynchrony. In each subject, the passive block experiment was conducted first, followed by the active event-related experiment. In the active experiment, letter-sound 
pairs were presented with different SOAs and the matching task had to be performed. Two different strategies were used to analyze the functional data from the second study. First, the passive runs were used to map ROIs in the auditory association cortex in which the congruency effect occurred. ROI-based statistics were performed on the active runs in the resulting ROIs (Analysis based on the passive experiment). Second, voxel-wise analyses were performed on the active runs, to further explore the effects of congruency during active integration within and beyond the auditory association cortex (Analysis based on the active experiment).

Analyses based on the passive experiment: The multi-subject congruency contrast performed on the passive block runs replicated the results of our previous studies (figure 4.5A): again, bilateral regions in auditory association cortex (see table 4.2 for details) showed the response pattern (Congruent $>$ Auditory $>$ Incongruent $>$ Visual), indicating cross-modal modulation of the response to speech sounds by simultaneously presented letters (Van Atteveldt et al., submitted [chapter 3]; Van Atteveldt et al., 2004 [chapter 2]). In contrast, the averaged time-courses and ROI-based statistics in the same regions demonstrated identical responses to congruent and incongruent stimuli at all three SOAs in the active experiment. As already indicated in study 1 , no effects of congruency or SOA seem to occur in the auditory association cortex when letters and sounds have to be integrated explicitly.

The congruency contrast on the group level resulted in one large ROI in each hemisphere (figure 4.5A). Because of the individual variability in functional neuroanatomy, potential congruency effects in the active experiment on the individual level may have disappeared in the group analysis because they were on different locations (i.e., in different voxels) within the large ROI. Therefore, the ROI-based group analysis of the active experiment may have lacked sensitivity to detect subtle differences present on the individual level, so we performed the congruency contrast on the passive runs also in single subjects to define individual ROIs in the auditory association cortex. The averaged Talairach coordinates of the resulting ROIs (table 4.2) were remarkably similar to the individual ROIs found for the same contrast in our previous study (Van Atteveldt et al., 2004 [chapter 2]). Figure 4.5B shows that also on the individual level, no effects of congruency and SOA were found in the active experiment.

Analyses based on the active experiment: The absence of the congruency effect in the active experiment could be due to restriction of the analyses to regions found by the passive experiment. This possibility was further investigated by performing wholebrain analyses on the active runs in addition to the ROI-based analyses. The congruency contrast in the active experiment revealed effects only in the direction incongruent $>$ congruent, located in different regions in the frontal and parietal cortex (figure 4.6A, table 4.3). The statistical map shown in figure 4.6A provides converging evidence for the absence of the congruency effect in the direction congruent $>$ incongruent in the auditory association cortex during active integration of letters and speech sounds.

In sum, studies 1 and 2 provide converging evidence for the significant effect of adding an explicit task on the neural mechanism for letter-speech sound integration: the congruency effect in the auditory association cortex, which was the key finding in our previous passive studies (Van Atteveldt et al., 2004 [chapter 2]), disappeared. Furthermore, the strong effect of temporal asynchrony in the auditory association cortex (Van Atteveldt et al., submitted [chapter 3]) was also absent during active 
integration. As was clear from the results of study 1 , the cancellation of the congruency and SOA effects was the result of enhancement of the response in the auditory cortex, especially in the incongruent condition. The lack of the effects of congruency and SOA indicates that the task demands changed the "default" response pattern in the auditory cortex. This may be explained in terms of top-down strategic control caused by the task instruction: the response to incongruent stimuli was enhanced because the task influenced the behavioral relevance of especially the incongruent stimuli.

\section{Frontal and parietal brain regions: source of top-down task effects?}

As mentioned before, the observed general enhancement of the response in the auditory cortex may be related to increased demands on attention during the active experiment (Coull, 1998; Hall et al., 2000). In the matching task, attention is not directed to one condition (congruent or incongruent) or modality (visual or auditory), both conditions and modalities are equally relevant for task performance. Attention differences between the active and passive tasks therefore probably reflect a general difference in the level of (cognitive) arousal or sustained/divided attention, instead of selective or directed attention (Coull, 1998). In addition to this increased level of general attention/arousal, the extra enhancement of the response to incongruent stimuli likely reflects a task-specific (strategic) top-down influence.

An interesting next question is where the influence of increased general attention/arousal and task-related strategic control on the auditory cortex originates. In other words, which network of higher-order brain regions is responsible for the taskrelated modulation of the "default" responses in the auditory cortex. This network should especially be active during the processing of incongruent stimulus pairs, since the response in the auditory cortex was mainly influenced by the task in this condition. Therefore, the activated frontal and parietal regions (figure 4.6, table 4.3) are plausible candidates for the source of the top-down modulation of activity in the auditory association cortex. The averaged time-courses of the response to congruent and incongruent trials in the revealed parietal and frontal regions (figure 4.6B) show significant activation in both conditions (except for the SPG), but stronger for incongruent trials. The SPG showed a somewhat different response pattern: this region was active exclusively during incongruent stimulation.

The postulated role for the revealed parietal and frontal regions in top-down taskrelated effects on the processing in sensory cortex, is consistent with the involvement of similar regions in attentional top-down control reported in the literature (Hopfinger et al., 2000; Posner and Dehaene, 1994), or more specifically, in goal-directed topdown selection of task-relevant stimuli and responses (Corbetta and Shulman, 2002). The ACG and IFS have been reported to be involved in divided attention (e.g. Corbetta et al., 1991). In the matching task of the present study, both visual and auditory modalities were equally relevant for the task and it was unpredictable in which modality the stimulus arrived first. The ACG and IFS may therefore be involved in keeping attention divided over the visual and auditory modalities.

In another study using speech stimuli, subjects also had to divide their attention over visual and auditory modalities and actively integrate written and spoken items (at the lexical level) (Shaywitz et al., 2001). The activated parietal and inferior frontal regions were suggested to be involved in processing potentially confusable linguistic tokens, which is consistent with the extra recruitment of these regions in the present study 
during incongruent letters and speech sounds. The involvement of superior and inferior parietal and superior frontal (premotor) cortices was also reported in a recent fMRI study on cross-modal matching of speech and lip movements (Saito et al., 2005). However, the parietal regions responded stronger to congruent than to incongruent stimuli, which was opposite to our findings.

The revealed frontal and parietal regions may also be related to working memory. During the asynchronous conditions of the letter-sound matching task, the overlearned paired associates have to be integrated cross-temporally. The higher activation of IFS and IPS during asynchronous trials (figure 4.6C), may be related to keeping the representation of the first stimulus active until the second arrives and matching can be performed. The response pattern of IFS and IPS therefore indicates that this activation may be the neural correlate of the faster reactions to asynchronously presented stimulus pairs (figure 4.3). This putative role of IFS and IPS in cross-modal and cross-temporal matching is consistent with findings from other studies (Fuster et al., 2000; Tanabe et al., 2005), although the SOAs in the present study were much shorter than typical delays used for revealing working memory-related brain areas.

To summarize, a frontal-parietal network was recruited during the cross-modal and cross-temporal matching of the letters and speech sounds, with strongest activation during the incongruent trials. This extra recruitment of attention and working memory related brain structures during the processing of incongruent stimuli suggests that these regions may be the origin of the top-down task effects on the auditory cortex: the disappearance of the congruency effect found during passive integration.

\section{Implications for the neural integration of letters and speech sounds}

In the two previous studies on letter-sound integration (Van Atteveldt et al., submitted [chapter 3]; Van Atteveldt et al., 2004 [chapter 2]), subjects passively perceived the stimuli without giving any responses. Since no top-down influences were exerted over the integration process, the previous findings reflect a default or automatic/mandatory integration mechanism for letters and speech sounds. In this default state, the brain only uses stimulus-related information (content/identity and temporal correspondence) in deciding which external stimuli belong together. Congruent and temporally coincident stimuli will be extracted as most relevant, because they provide corresponding information about the same external event. On the contrary, incongruent and asynchronously presented combinations of letters and speech sounds are extracted as not belonging together, and therefore less relevant (Driver and Spence, 2000; Lalanne and Lorenceau, 2004). As the presumed integration site that determines congruency and temporal relation, the STS/STG may play an important role in signaling this relevance (Van Atteveldt et al., 2004 [chapter 2]; Van Atteveldt et al., submitted [chapter 3]). The processing of speech sounds in the auditory cortex is enhanced or suppressed according to the relevance values assigned by the integration process. In this interpretation, the congruency effect in the auditory cortex reflects the result of a default integration process.

In the matching task during the active experiments in the present study, subjects had to make a same/different decision after each stimulus pair. This task instruction changed the behavioral relevance of the stimuli compared to the passive/default situation. Because a decision is required after each stimulus, all stimuli are equally relevant, independent of congruency and temporal offset. Therefore, the (bottom-up) default 
selection of relevant and irrelevant information is overruled by (top-down) task demands. This mainly affects incongruent stimuli, being relevant during active integration, but irrelevant when binding is only based on bottom-up processing. The absence of the effects of the stimulus properties (congruency and temporal relation) in the auditory cortex during the active task supports the idea that the default (bottom-up) integration process is overruled by top-down strategic control during the explicit letterspeech sound matching task. The revealed frontal-parietal network may serve as the source of this top-down strategic control.

\section{General implications}

The finding that changing the task from passive viewing/listening to active matching of the stimuli overruled the stimulus-related integration effects points out how rigorous top-down effects on multisensory integration can be. Therefore, this study provides support for the notion that laboratory tasks contaminate mandatory perceptual processes by inducing post-perception response strategies and decision processes (de Gelder and Bertelson, 2003). This is an important aspect to keep in mind when designing cross-modal experiments.

\section{Limitations of the present study}

Because we did not include within-modality matching conditions (V-V and A-A), the matching task as employed in the present study is not suitable to reveal the brain mechanism specialized for cross-modal matching, like in other studies (Banati et al., 2000; Hadjikhani and Roland, 1998; Saito et al., 2005; Saito et al., 2003). However, for the main goal of the present study, which was to compare response patterns between passive and active integration in prespecified brain regions, within-modality matching conditions were not necessary and would therefore only unnecessarily have increased the duration of the experiments.

Another limitation of the present study is that for comparing active and passive integration, it was limited to the bimodal presentation conditions. Another region of high interest, the heteromodal superior temporal cortex (STS/STG) was identified in our previous studies with a conjunction of the contrasts: (bimodal $>$ unimodal) $\cap$ (unimodal $>$ baseline). In the active experiments of the present study, only bimodal stimuli were presented, eliminating the possibility to search for regions showing enhanced responses to bimodal stimuli as compared to unimodal stimuli. To investigate the effect of top-down task-related factors on the integrative role of the STS/STG, other experiments will be necessary using a different behavioral task, e.g. including withinmodality matching conditions.

Finally, the comparison of passive and active integration might have been more direct if an event-related PMRI design would have been used in the passive experiment in study 2 . However, for achieving equal statistical power, the duration of slow eventrelated runs are much longer than that of block runs. As our previous studies showed the robustness and reproducibility of the congruency effect using a passive block design, and the congruency effect was replicated using an event-related design in the first study, we chose to use the passive block experiment in study 2 to confidently map regions involved in passive integration. 


\section{Conclusions}

The principle results of the present study are:

1. We replicated the congruency effect in the auditory association cortex found in our previous studies (Van Atteveldt et al., submitted [chapter 3]; Van Atteveldt et al., 2004 [chapter 2]) with a different group of subjects and different experimental designs;

2. The congruency effect in the auditory association cortex cannot be explained by attention or arousal due to the blocked stimulus presentation, because the effect was replicated with random stimulus presentation;

3. An event-related fMRI design is suitable for studying integration of letters and speech sounds, since differential responses to single letters, speech sounds and their (congruent or incongruent) combinations can be detected;

4. The effects of congruency and temporal asynchrony in the auditory association cortex were absent during active integration, indicating that top-down task effects overruled the "default" (bottom-up) integration of letters and speech sounds. Because of the task instruction, both congruent and incongruent stimulus pairs were behaviorally relevant, which resulted in the disappearance of the congruency effect in the auditory cortex.

5. The source of this top-down task effect is suggested to be a frontal-parietal network, reflecting a combination of attention and working memory related brain areas, controlling the explicit cross-modal and cross-temporal integration of the overlearned associations between letters and speech sounds. 


\section{References}

Amedi, A., von Kriegstein, K., Van Atteveldt, N. M., Beauchamp, M. S., and Naumer, M. J. (2005). Functional imaging of human crossmodal identification and object recognition. Experimental Brain Research 166, 559-571.

Andersen, T. S., Tiippana, K., and Sams, M. (2004). Factors influencing audiovisual fission and fusion illusions. Cognitive Brain Research 21, 301-308.

Banati, R. B., Goerres, G. W., Tjoa, C., Aggleton, J. P., and Grasby, P. (2000). The functional anatomy of visual-tactile integration in man: a study using positron emission tomography. Neuropsychologia 38, 115-124.

Beauchamp, M. (2005). See me, hear me, touch me: multisensory integration in lateral occipitaltemporal cortex. Current Opinion in Neurobiology 15, 1-9.

Boynton, G. M., Engel, S. A., Glover, G. H., and Heeger, D. J. (1996). Linear Systems Analysis of Functional Magnetic Resonance Imaging in Human V1. Journal of Neuroscience 16, 42074241.

Calvert, G. A. (2001). Crossmodal Processing in the Human Brain: Insights from Functional Neuroimaging Studies. Cerebral Cortex $11,1110-1123$.

Calvert, G. A., Campbell, R., and Brammer, M. J. (2000). Evidence from functional magnetic resonance imaging of crossmodal binding in the human heteromodal cortex. Current Biology J0, 649-657.

Corbetta, M., Miezin, F. M., Dobmeyer, S., Shulman, G. L., and Petersen, S. E. (1991). Selective and divided attention during visual discriminations of shape, color, and speed: functional anatomy by positron emission tomography. Journal of Neuroscience 11, 2383-2402.

Corbetta, M., and Shulman, G. L. (2002). Control of goal-directed and stimulus-driven attention in the brain. Nature Reviews Neuroscience 3, 201-215.

Coull, J. T. (1998). Neural correlates of attention and arousal: insights from electrophysiology, functional neuroimaging and psychopharmacology. Progress in Neurobiology 55, 343-361.

de Gelder, B., and Bertelson, P. (2003). Multisensory integration, perception and ecological validity. Trends in Cognitive Sciences 7, 460-467.

Donaldson, D. I., and Buckner, R. L. (2001). Effective paradigm design. In Functional MRI: an introduction to the methods, P. Jezzard, P. M. Matthews, and S. M. Smith, eds. (Oxford, Oxford University Press).

Driver, J., and Spence, C. (2000). Multisensory perception: Beyond modularity and convergence. Current Biology 10, R731-R735.

Duvernoy, H. M. (1999). The Human Brain: surface, three-dimensional sectional anatomy with MRI, and blood supply, Second, completely revised and enlarged edition (Wien New York, Springer-Verlag)

Ehri, L. C. (2005). Development of sight word reading: phases and findings. In The Science of Reading: A Handbook, M. J. Snowling, and C. Hulme, eds. (Oxford, Blackwell publishing).

Fuster, j. m., Bodner, m., and Kroger, j. k. (2000). Cross-modal and cross-temporal association in neurons of frontal cortex. Nature 45, 347-351.

Grady, C. L., Van Meter, J. W., Maisog, J. M., Pietrini, P., Krasuski, J., and Rauschecker, J. P. (1997). Attention-related modulation of activity in primary and secondary auditory cortex. Neuroreport 8, 2511-2516.

Hadjikhani, N., and Roland, P. E. (1998). Cross-Modal Transfer of Information between the Tactile and the Visual Representations in the Human Brain: A Positron Emission Tomographic Study. The Journal of Neuroscience 18, 1072-1084.

Hall, D. A., Haggard, M. P., Akeroyd, M. A., Summerfield, A. Q., Palmer, A. R., Elliott, M. R., and Bowtell, R. W. (2000). Modulation and task effects in auditory processing measured using fMRI. Human Brain Mapping 10, 107-I 19. 
Hopfinger, J. B., Buonocore, M. H., and Mangun, G. R. (2000). The neural mechanisms of top-down attentional control. Nature Neuroscience 3, 284-291.

Jäncke, L., Wüstenberg, T., Scheich, H., and Heinze, H. J. (2002). Phonetic Perception and the Temporal Cortex. Neurolmage 15, 733-746.

King, A. J., and Calvert, G. A. (2001). Multisensory integration: perceptual grouping by eye and ear. Current Biology 11 , R322-R325.

Lalanne, C., and Lorenceau, J. (2004). Crossmodal integration for perception and action. Journal of Physiology - Paris 98, 265-279.

National Reading Council (1998). Preventing reading difficulties in young children (Washington, DC, National Academy Press).

Posner, M., and Dehaene, S. (1994). Attentional networks. Trends in Neuroscience 17, 1994.

Saito, D., Yoshimura, K., Kochiyama, T., Okada, T., Honda, M., and Sadato, N. (2005). Cross-modal binding and activated attentional networks during audiovisual speech integration: a functional MRl study. Cerebral Cortex 5, 1750-1760.

Saito, D. N., Okada, T., Morita, Y., Yonekura, Y., and Sadato, N. (2003). Tactile-visual cross-modal shape matching: a functional MRI study. Cognitive Brain Research 17, 14-25.

Savoy, R. L., Bandettini, P. A., O'Craven, K. M., Kwong, K. K., Davis, T. L., Baker, J. R., Weiskoff, R. M., and Rosen, B. R. (1995). Pushing the temporal resolution of fMRI: Studies of very brief visual stimuli, onset vriability and asycnhrony, and stimulus-correlated changes in noise. Proceedings of the Society of Magnetic Resonance Third Scientific Meeting and Exhibition 2, 450.

Shaywitz, B. A., Shaywitz, S. E., Pugh, K. R., Fulbright, R. K., Skudlarski, P., Mencl, W. E., Constable, R. T., Marchione, K. E., Fletcher, J. M., Klorman, R., et al. (2001). The Functional Neural Architecture of Components of Attention in Language-Processing Tasks. Neurolmage $13,601-612$.

Tanabe, H. C., Honda, M., and Sadato, N. (2005). Functionally segregated neural substrates for arbitrary audiovisually paired-association learning. The Journal of Neuroscience 25,64096418.

Van Atteveldt, N., Formisano, E., Blomert, L., and Goebel, R. (submitted). The effect of temporal asynchrony on the multisensory integration of letters and speech sounds.

Van Atteveldt, N., Formisano, E., Goebel, R., and Blomert, L. (2004). Integration of letters and speech sounds in the human brain. Neuron 43, 271-282.

Vellutino, F. R., Fletcher, J. M., Snowling, M. J., and Scanlon, D. M. (2004). Specific reading disability (dyslexia): what have we learned in the past four decades? Journal of Child Psychology and Psychiatry 45, 2-40. 


\section{Chapter 5}

\section{General Discussion}

This chapter is partly based on:

Amedi, A.*, von Kriegstein, K.*, Van Atteveldt, N. M.*, Beauchamp, M. S.*, and Naumer, M. J.* (2005). Functional imaging of human crossmodal identification and object recognition. Experimental Brain Research 166, 559-571.

*All authors contributed equally to this work 


\subsection{Summary and discussion of the main findings}

\section{Summary}

In chapter 2, a functional neuro-anatomical mechanism for the passive integration of simultaneously presented letters and speech sounds was revealed (see figure 2.5). Modality-specific processing was observed in superior temporal and occipital-temporal cortices, convergence and integration in the heteromodal STS/STG. A most interesting finding was that subregions of the posterior auditory association cortex involved in speech sound processing, located on Heschl's sulcus (HS) and the planum temporale (PT), were influenced by the congruency of simultaneously presented letters. Because these regions in HS and PT did not respond to letters when presented in isolation, we interpreted this influence as feedback modulation from heteromodal STS/STG regions where integration took place.

In chapter 3, the relative importance of temporal proximity and information content (identity congruency) on the passive integration of letters and speech sounds was investigated, by manipulating both factors within the same fMRI design. The effect of congruency in the posterior auditory association cortex (PT) found in chapter 2 was replicated, and was also found in the anterior auditory association cortex (anterior superior temporal plane, aSTP). The results furthermore revealed significant interactions between temporal proximity and content congruency in PT and aSTP, which indicated that temporal synchrony was critical for the integration of letters and speech sounds. Interestingly, multisensory interactions in the STS were observed over a broader temporal range.

In chapter 4, the effects of stimulus presentation mode and top-down task-related factors on the neural integration of letters and speech sounds were examined. Based on the results of chapters 2 and 3, the effects of congruency and temporal asynchrony in the auditory association cortex were further explored in a series of experiments in which stimulus presentation mode and task demands were systematically manipulated. Using event-related fMRI, we replicated the congruency effect in the PT that was found with block designs in chapters 2 and 3 . This excluded the possible explanation of the congruency effect in terms of attention differences caused by the blocked stimulus presentation. A second important finding was the disappearance of the effects of congruency and temporal proximity in the PT during active matching of letters and speech sounds. This indicated that the task demands overruled the automatic/default multisensory responses to letters and speech sounds, most likely because the task changed the behavioral relevance of the stimuli. The source of this top-down task effect was suggested to be a frontal-parietal network that controls explicit matching of the over-learned associations between letters and speech sounds.

\section{A functional neuro-anatomical mechanism for the integration of letters and speech sounds}

Based on the findings of chapters 2 and 3, we propose the following neural mechanism for the automatic integration of letters and speech sounds: speech sounds are primarily represented and processed in the PT (Griffiths and Warren, 2002; Hickok and Poeppel, 2000 ). The next processing level, the STS, also receives visual information about letters, and integrates both inputs within a broad range of SOAs. Depending on the temporal relationship between the inputs from both modalities, information regarding 
identity congruency is projected back to the auditory association cortex, resulting in the observed temporal profiles of multisensory interaction. In the passive viewing and listening situation of the studies in chapters 2 and 3, basic rules of temporal proximity seem to apply to the automatic binding of letters and speech sounds, and feedback to the auditory association cortex seems only to be provided when the stimuli are presented in temporal synchrony.

In the studies reported in chapters 2 and 3, no top-down influences were exerted over the integration process because subjects passively perceived the stimuli without giving any responses. Therefore, these findings reflect a default or automatic/ mandatory integration mechanism for letters and speech sounds. In this default state, the brain mainly uses stimulus-related information (content/identity and temporal correspondence) in deciding which external stimuli belong together. Congruent and temporally coincident stimuli provide corresponding information about the same external event and will therefore be extracted as most relevant. On the contrary, incongruent and asynchronously presented combinations of letters and speech sounds are extracted as not belonging together, and therefore most likely as less relevant. In chapter 2 and 3, the STS was advanced as integration site that determines the content congruency and temporal relation and may therefore play an important role in the automatic signaling of the relevance of passively perceived audiovisual stimuli. The processing of speech sounds in the auditory cortex is enhanced or suppressed according to the relevance values assigned by the integration process. In this interpretation, the congruency effect in the auditory association cortex reflects the result of a default or automatic integration process.

In the active task in chapter 4, subjects had to make a same/different decision after each stimulus pair. During this explicit matching, the congruency effect in the auditory association cortex was absent. This suggests that the response to letter-sound pairs in the auditory association cortex is depending on the situation (task at hand). The task instruction (chapter 4) changed the behavioral relevance of the stimuli compared to the passive/default situation (chapters 2 and 3). Because a decision was required after each stimulus, all stimuli were equally relevant, independent of congruency and temporal offset. Therefore, the (bottom-up) default selection of relevant and irrelevant information was overruled by (top-down) task demands. A network of frontal-parietal brain regions was suggested as the source of this top-down strategic control.

\section{Unidirectionality of the congruency effect}

The effects of congruency and temporal asynchrony observed in the auditory association cortex (chapters 2 and 3, and passive experiments in chapter 4) were not observed in the visual association cortex. This implies that the outcome of the (passive) integration of letters and speech sounds is projected back to influence only early auditory processing levels, but not lower-level visual processing. In chapter 2 , it was discussed that the direction of the modulatory effects between letters and speech sounds may depend on the temporal synchrony of the stimuli. This possibility was excluded in chapter 3, with the finding that cross-modal modulation of responses to letters in the visual association cortex were also absent when letter-sound pairs were presented at different temporal offsets (in both directions). This finding makes it unlikely that the absence of feedback to the visual cortex is due to timing differences within the processing streams of letters and speech sounds. 
The observed unidirectional influence may reflect the parasitic nature of written language (see $\$ 1.1$ ). Since written language parasites on spoken language and not the other way around, it seems plausible that the visual information travels to the speech processing system and not vice versa. Another possible explanation assumes differences in processing difficulty. Letters are discrete characters with a high signalto-noise ratio. Speech sounds are continuous in time and more variable, and therefore more difficult to recognize (Liberman, 1992). According to the modality appropriateness theory (Welch and Warren, 1980), the modality that provides the most accurate/reliable information dominates the multisensory integration process, e.g. by influencing the other modality. This is consistent with the finding of Sekiyama and colleagues (2003) that visual information exerts a stronger influence on auditory processing when speech is less intelligible. Another hypothesis that seems relevant in this context is the discontinuity hypothesis (Shams et al., 2002) which states that the influence of the discontinuous modality on the continuous modality is stronger than vice versa.

The unidirectional influence of letters on speech sound processing may furthermore be explained from a developmental perspective. During reading acquisition, phoneme processing/awareness and literacy skills are reciprocally related. Learning the letters of the alphabet and the letter-sound mappings strongly influences the processing and representation of single phonemes (Morais and Kolinsky, 2005). This may explain the influence of letters on the processing of isolated speech sounds as observed by the congruency effect in this thesis.

\section{Congruency effect: feedback or feedforward?}

In accordance with other studies advocating a feedback mechanism for audiovisual integration of speech information (Calvert et al., 2000; King and Calvert, 2001), we interpreted the observed congruency effect in the auditory cortex as a feedback modulatory influence from the STS/STG. This idea is consistent with a more general view of distributed semantic processing. In this view, multisensory regions are presumed to interact with unisensory cortices via feedback connections, thereby constituting a cortical network in which semantic knowledge about objects is represented in a distributed fashion (Martin and Chao, 2001). According to this model, brain regions that process a specific type of incoming sensory information also serve as a knowledge store about the attributes of particular objects. For instance, networks of neurons in auditory association cortex that respond to an auditory object are also linked to visual, tactile, and motor information about the same object represented in occipitaltemporal, parietal, and frontal regions. Mesulam (1998) suggests that multisensory associative areas contain "road maps" for binding distributed information in different modalities. In this view, associative areas do not contain multimodal representations but merely link the unimodal representations to make them accessible to each other. Contrary to the view of heteromodal associative or integration regions providing feedback to unisensory cortices, recent animal studies have provided evidence for direct anatomical connections between sensory cortices (Falchier et al., 2002; Rockland and Ojima, 2003), indicating the possibility of direct interactions. Furthermore, recent neuroimaging studies have provided support on a functional level for direct interactions between sensory cortices during multisensory integration without involvement of heteromodal areas (e.g. von Kriegstein et al., 2005). A possible explanation for the 
diverse findings is that the involvement of multisensory associative regions may be dependent on the type of information that has to be bound together.

This view is supported by findings of different types of anatomical connectivity (feedforward vs. feedback) between unisensory and multisensory areas in primates (Schroeder and Foxe, 2005). For example, Schroeder and Foxe (2002) investigated the laminar profile and response latencies of visual and somatosensory input in the posterior auditory association cortex of the macaque monkey. The laminar input profile and response latencies indicated that visual input was of feedback type, while the properties of somatosensory inputs suggested feedforward connections. The feedback nature of the visual input may indicate that integration of auditory and visual inputs is involved in more complex functions requiring more detailed analysis of the incoming information (e.g. audiovisual speech perception). Schroeder and Foxe (2002) suggest that feedback dependent processes may be important for the involvement of low-level sensory areas in conscious experience of stimuli. On the other hand, somatosensory input is suggested to be involved in orienting and localization functions, based on the finding that the input mainly originates from cutaneous representations of the head and neck (Fu et al., 2003). For such functions, it is crucial that integration of incoming information from different senses is fast. This is consistent with the suggested role of direct connections between auditory and visual primary cortices in motion perception and/or alerting responses (Falchier et al., 2002; Rockland and Ojima, 2003). These examples suggest that direct sharing of information between modalities, without recruitment of multisensory regions, seems to be found for information that needs to be identified quickly and automatically (alerting, orienting, and localizing responses, motion perception). On the contrary, more complex or arbitrarily related information (e.g. audiovisual speech, letters, and common objects) might be integrated through associative nodes (e.g. STS) that are able to form more complex and flexible mappings between information from different modalities.

In sum, modality-specific representations in unisensory association cortices are linked either directly or through a heteromodal binding site, depending on the information type and goal or the requirements of the crossmodal combination. In the case of letters and speech sounds, the artificial nature and flexible use of the associations makes the involvement of the STS as heteromodal binding site plausible. On the other hand, we cannot exclude the possibility that the planum temporale itself serves as associative node, which links visual, auditory and motor representations of letters (see chapter 3 ).

\subsection{Implications of the proposed functional neuro-anatomical mechanism for letter-sound integration}

As stated in the introduction, the proposed functional neuro-anatomical mechanism for letter-speech sound integration directly contributes to a better understanding of the neural basis of alphabetic reading (literacy) and cross-modal neural processing. In addition, the results that are reported in this thesis may have more indirect implications for future studies concerning literacy acquisition, developmental dyslexia and the biological validation of training effects. These direct and indirect implications will be discussed in this section. 


\section{Neural basis of alphabetic reading}

The results of the experiments presented in this thesis indicate that in the normally developed, highly automated system, letters and speech sounds are automatically associated/ integrated when they are perceived. The result of the automatic integration process in heteromodal regions of the superior temporal cortex (STS/STG) is projected back to subregions of the posterior (PT, chapters 2-4) and anterior (aSTP, chapter 3) auditory association cortex. Here, speech sound processing is enhanced or suppressed depending on the congruency of the letter-sound pair (congruency effect). Furthermore, the congruency effect in the auditory association cortex was found to be critically dependent on the temporal synchrony of the letters and speech sounds and the passive nature of the integration process. The proposed neuro-anatomical mechanism and the demonstrated effects of stimulus- and task-related factors provide a working model for how letters and speech sounds are associated in literate adults.

\section{Cross-modal information processing}

The findings furthermore increase fundamental insights in how the human brain integrates artificially related multisensory inputs. A first new insight is provided by the revealed narrow temporal windows for multisensory integration in the auditory association cortex in chapter 3. The temporal characteristics of the integration responses strongly resembled those demonstrated for single multisensory neurons in different brain structures and animal species (Meredith et al., 1987; Stein and Wallace, 1996; Wallace et al., 1996). This similarity suggests that basic neural integration rules also apply to the binding of multisensory information that is not naturally related, but over-learned during literacy acquisition.

The demonstrated narrow temporal windows furthermore support the idea that the importance of temporal correspondence is depending on the nature and complexity of the multisensory stimuli (see \$1.2). Calvert and colleagues (Calvert et al., 1998) have suggested that simultaneous onset is a strong binding factor for simple multisensory stimuli with little shared features. More complex stimuli have more shared content, so simultaneous onset is less important. Our results indicate that letter-sound associations seem to behave like the first example, most likely because they lack naturally corresponding properties. On the contrary, temporal coherence appears to be only loosely important for audiovisual speech signals to be integrated (Massaro and Cohen, 1993; Massaro et al., 1996; Munhall et al., 1996; Munhall and Vatikiotis-Bateson, 2004). This fits with the fact that audiovisual speech signals have more inherently corresponding features, such as frequency-amplitude information (Calvert, 2001; Munhall and Vatikiotis-Bateson, 1998). To conclude, the importance of temporal proximity seems to be more important for integration of the artificially related letters and speech sounds, than for integration of the naturally related audiovisual speech signals.

A second insight related to cross-modal information processing is provided by the task effects reported in chapter 4 . Changing the task instruction from passive viewing/listening to active matching overruled the stimulus-related integration effects, which indicates how rigorous top-down task effects on multisensory integration can be. This is an important aspect to keep in mind when designing cross-modal experiments. 


\section{Development of letter-sound associations}

The robustness and reproducibility of our findings on passive integration of letters and speech sounds (especially the congruency effect in the PT) support the use of the proposed working model as a basis for investigating normal and abnormal development of letter-sound associations. An interesting next question is how letter-sound associations normally develop and when they become fully automated. Furthermore, the working model can be used to test the hypothesis that the development of lettersound mappings is deviant in developmental dyslexics, and whether this is caused by an allophonic mode of speech perception.

An important implication of the experiments reported in chapter 4 is that while the passive experiments are suitable to compare automatic integration between different reading and age groups, the active matching task is less suitable because the integration mechanism is less clear. In developmental imaging studies however, it is strongly recommended to use an active paradigm. A behavioral measure keeps track of what subjects were doing and understanding during the functional brain measurements. Without such a measure, the origins of possible group differences in brain activation are difficult to interpret (Casey et al., 2005). More research is needed to select suitable tasks for this purpose.

\section{Biological validation of training effects}

In $\S 1.1$, it was mentioned that training programs are able to improve reading ability, but are most effective if they include letter-sound training. Insights in the neural substrates of such improvements are of major importance (Habib, 2003). If a training program is effective in improving reading skills, it is important to establish the changes in recruited brain areas accompanying the training effects on the behavioral level. Only in this way, it can be determined whether the observed effects are the result of functional brain changes in the direction of the normal pattern.

Several studies have already reported plastic brain changes after relatively short periods of training (Eden et al., 2004; Kujala et al., 2001; Richards et al., 2000; Temple et al., 2003), or even the reversal of the deviant functional brain organization (Simos et al., 2002). However, these studies did not measure changes in neural activation during letter-sound processing. The experiments presented in this thesis provide suitable experimental paradigms for testing letter-sound integration in the brain before and after a period of training.

\subsection{Similar neural mechanisms for reading and lip reading?}

In the general introduction, it was speculated that a neural foundation provided by the evolutionary adapted brain mechanism for audiovisual speech integration may explain the fast learning and efficient use of the culturally defined letter-sound mappings. Although we did not address this hypothesis directly in the present experiments, a comparison of the present fMRI results to what is reported in the literature on audiovisual speech integration may indicate indirectly whether similar neural mechanisms might be involved. 


\section{Audiovisual speech versus letter-speech integration}

A talking person emits both speech sounds and corresponding visible facial movements. Therefore, mouth movements of a speaker form a natural visual component of speech perception. Mouth movements and phonetic information are inherently linked by characteristics such as temporal onset, duration and frequencyamplitude information (Calvert, 2001; Munhall and Vatikiotis-Bateson, 1998). Correspondences between speech sounds and mouth movements are learned implicitly and early in development, by exposure to heard speech together with the sight of the speaker (Kuhl and Meltzoff, 1982). In contrast, the visual representation of spoken language by written language is an artificial, cultural invention. Letters and speech sounds exhibit no natural corresponding characteristics but are related in an arbitrary way. Therefore, associations between letters and speech sounds are not learned automatically but require explicit instruction (Gleitman and Rozin, 1977; Liberman, 1992).

Viewing the face of a speaker during listening enhances speech perception, especially under noisy conditions (Sumby and Pollack, 1954). Lip reading can also change the speech percept, which is illustrated by the McGurk effect (McGurk and MacDonald, 1976). A possible neural basis for these perceptual effects is the activation of auditory processing regions by silent lip reading, as is reported by several fMRI studies (Bernstein et al., 2002; Calvert et al., 1997; Olson et al., 2002; Paulesu et al., 2003; Sekiyama et al., 2003). Consistently reported auditory regions include the STS, STG, and auditory association regions like the planum temporale that surround the primary auditory cortex (A1). The involvement of A1, as reported by Calvert et al. (1997), is a matter of debate (Bernstein et al. 2002; Paulesu et al. 2003).

In addition to studies focusing on the visual component of audiovisual speech, the integration of auditory and visual speech has also been addressed directly. Despite wide variations in methodology, a consistent finding from audiovisual speech studies is the involvement of the left superior temporal sulcus (STS) and middle/superior temporal gyri (MTG/STG) in the integration of heard and seen speech (Callan et al., 2003; Calvert et al., 2000; Macaluso et al., 2004; Sekiyama et al., 2003; Wright et al., 2003), see also figure 5.1). Interestingly, crossmodal effects in the STS seem to be strongest when the auditory information is degraded by noise (Callan et al., 2001; Callan et al., 2003; Sekiyama et al., 2003), which is consistent with earlier behavioral findings (Sumby and Pollack, 1954). A mechanism for audiovisual speech perception has been proposed in which the auditory and visual speech signals are integrated in STS/STG, followed by modulation of the relevant unisensory cortices (auditory association cortex and MT/V5) via feedback projections (Calvert et al., 1999; Calvert et al., 2000).

The neural basis of the associations between speech sounds and their written representations, letters, is much less extensively investigated (see figure 5.1). In the fMRI study presented in chapter 2 (Van Atteveldt et al., 2004), several bilateral STS/STG regions were identified that responded stronger to bimodal congruent letters as compared to their respective unimodal components. Furthermore, the response to speech sounds in early unisensory auditory regions in Heschl's sulcus (HS) and planum temporale (PT) was found to be modulated by the degree of congruency with a simultaneously presented letter. Because these early auditory regions did not respond to unimodal visual letters, we interpreted the modulation as a feedback effect from 
integration sites in the STS/STG. An MEG study provided converging time course information for the processing and integration of letters and speech sounds, and the estimated source localizations in this study also indicated the involvement of STS in the integration of letters and speech sounds (Raij et al., 2000). More evidence for this function of the STS was provided by a recent learning experiment (Hashimoto and Sakai, 2004), in which it was found that the STG/MTG was active during processing of already acquired matching letter-sound combinations.

The post-hoc comparison of neuroimaging results on audiovisual speech versus letterspeech integration indicates that the recruited brain structures and potential feedback mechanism seem similar during cross-modal integration of both types of associations. The overlap of activated superior temporal regions in various neuroimaging studies on audiovisual speech as well as letter-speech sound integration is shown in figure 5.1.

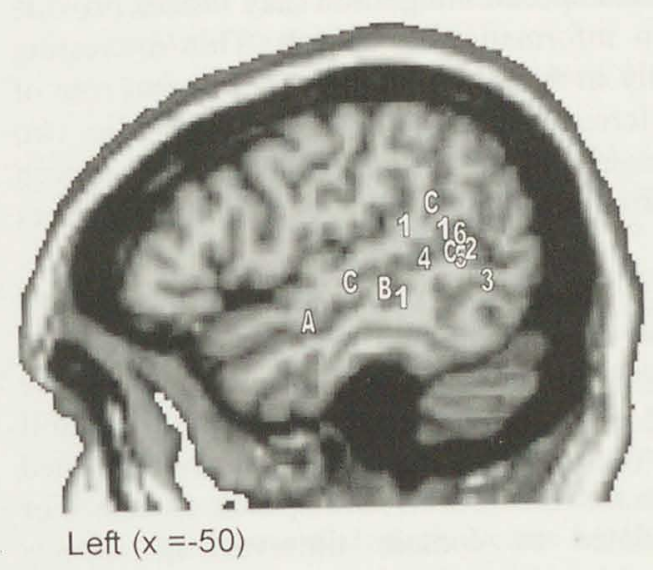

Audiovisual speech:

1) Callan et al. 2003

2) Calvert et al. 2000

3) Macaluso et al. 2003

4) Olson et al. 2002

5) Sekiyama et al. 2003

6) Wright et al. 2003

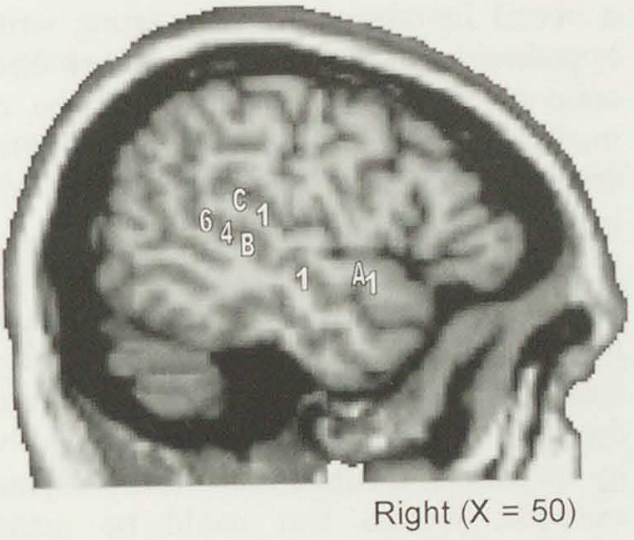

Letters and speech sounds:
A) Hashimoto et al. 2004
B) Raij et al. 2000
C) Van Atteveldt et al. 2004

Figure 5.1 A summary of the reported superior temporal regions (mainly STS, MTG and STG) involved in integration of audio-visual language information. The most important activations of the reviewed studies are projected in sagittal slices of the MNI template brain transformed into Talairach space. LH is shown at $x=-50, R H$ at $x=50$. Circles reflect results from studies using speech and lip movement stimuli (i.e. naturally related audio-visual stimuli), stars reflect results from studies using letters and speech sounds (artificially related audio-visual stimuli). 
However, as already discussed in $\S 5.2$, an important difference between audiovisual speech and letter-speech integration is the importance of temporal proximity. Audiovisual speech integration is known to be relatively unaffected by temporal asynchrony (Massaro and Cohen, 1993; Massaro et al., 1996; Munhall et al., 1996; Munhall and Vatikiotis-Bateson, 2004), while our findings indicated strong temporal constraints for the integration of letters and speech sounds. This apparent discrepancy may be explained by the fact that in audiovisual speech, the visual and auditory inputs share more features, e.g. time-varying aspects such as frequency-amplitude information (Calvert et al., 1998; Munhall et al., 1996; Munhall and Vatikiotis-Bateson, 1998). Because letters and speech sounds lack these naturally corresponding features, it is tentative to assume that simultaneous onset is more critical for their integration.

\section{Implications for learning to read}

The resemblance of the neural mechanisms for letter-speech integration provided by the present experiments to that for audiovisual speech integration reported by other neuroimaging studies indicates that audiovisual speech integration may indeed provide a neural foundation for associating written information to speech. This interesting hypothesis should be addressed more directly in future research. The different role of temporal proximity might reflect the difference in naturalness between the two instances of audiovisual associations. Because of this difference, letters and speech sounds exhibit less inherently shared information, which may explain the stronger importance of temporal proximity.

These observations may lead to the following speculative suggestion: if it would indeed be the case that the neural mechanism for audiovisual speech integration provides a neural foundation for letter-speech sound integration, the use of the audiovisual speech processing system during reading acquisition might be optimized if the cross-modal learning process would be more natural. This could be accomplished by increasing the amount of shared features between letters and speech sounds. For example, written text could be manipulated to contain time-varying aspects corresponding to the speech signal, e.g. size or intensity of the letters corresponding to the amplitude of the speech signal, and continuous transition between letters timelocked to the transitions between phonemes in the speech signal. In designing this "natural script", information from lip movements may be used. In this way, the learning process could be made more "natural", and the use of the evolutionary adapted brain system for audiovisual speech may be optimized. 


\section{References}

Bernstein, L. E., Auer, E. T., Moore, J. K., Ponton, C. W., Don, M., and Singh, M. (2002). Visual speech perception without primary auditory cortex activation. Neuroreport 13, 311-315.

Bertelson, P., and de Gelder, B. (2004). The psychology of multimodal perception. In Crossmodal space and crossmodal attention, C. Spence, and J. Driver, eds. (Oxford, Oxfor University Press).

Callan, D. E., Callan, A. M., Kroos, C., and Vatikiotis-Bateson, E. (2001). Multimodal contribution to speech perception revealed by independent component analysis: a single-sweep EEG case study. Cognitive Brain Research 10, 349-353.

Callan, D. E., Jones, J. A., Munhall, K., Callan, A. M., Kroos, C., and Vatikiotis-Bateson, E. (2003). Neural processes underlying perceptual enhancement by visual speech gestures. NeuroReport $14,2213-2218$.

Calvert, G. A. (2001). Crossmodal Processing in the Human Brain: Insights from Functional Neuroimaging Studies. Cerebral Cortex 11, $1110-1123$.

Calvert, G. A., Brammer, M. J., Bullmore, E. T., Campbell, R., Iversen, S. D., and David, A. S. (1999). Response amplification in sensory-specific cortices during crossmodal binding. Neuroreport 10,2619-2623.

Calvert, G. A., Brammer, M. J., and Iversen, S. D. (1998). Crossmodal identification. Trends in Cognitive Sciences 2, 247-253.

Calvert, G. A., Bullmore, E. T., Brammer, M. J., Campbell, R., Williams, S. C., McGuire, P. K., Woodruff, P. W., Iversen, S. D., and David, A. S. (1997). Activation of auditory cortex during silent lipreading. Science 276, 593-596.

Calvert, G. A., Campbell, R., and Brammer, M. J. (2000). Evidence from functional magnetic resonance imaging of crossmodal binding in the human heteromodal cortex. Current Biology 10, 649-657.

Casey, B. J., Tottenham, N., Liston, C., and Durston, S. (2005). Imaging the developing brain: what have we learned about cognitive development? Trends in Cognitive Sciences 9, 104-110.

Eden, G. F., Jones, K. M., Cappell, K., Gareau, L., Wood, F. B., Zeffiro, T. A., Dietz, N. A., Agnew, J. A., and Flowers, D. L. (2004). Neural changes following remediation in adult developmental dyslexia. Neuron 44, 41 1-422.

Falchier, A., Clavagnier, S., Barone, P., and Kennedy, H. (2002). Anatomical Evidence of Multimodal Integration in Primate Striate Cortex. Journal of Neuroscience 22, 5749-5759.

Fu, K., Johnston, T., Shah, A., Arnold, L., Smiley, J., Hackett, T., Garraghty, P., and Schroeder, C. (2003). Aduitory cortical neurons respond to somatosensory input. Journal of Neuroscience $23,7510-7515$.

Gleitman, L. R., and Rozin, P. (1977). The structure and acquisition of reading I: relations between orthographies and the structure of language. In Towards a psychology of reading: the proceedings of the CUNY conferences, A. Reber, and D. Scarborough, eds. (Hillsdale, N.J, Lawrence Erlbaum Associates).

Griffiths, T. D., and Warren, J. D. (2002). The planum temporale as a computational hub. Trends in Neuroscience 25, 348-353.

Habib, M. (2003). Rewiring the dyslexic brain. Trends in Cognitive Sciences 7, 2003.

Hashimoto, R., and Sakai, K. L. (2004). Learning letters in adulthood: direct visualization of cortical plasticity for forming a new link between orthography and phonology. Neuron 42, 311-322.

Hickok, G., and Poeppel, D. (2000). Towards a functional neuroanatomy of speech perception. Trends in Cognitive Sciences 4, 131-138.

King, A. J., and Calvert, G. A. (2001). Multisensory integration: perceptual grouping by eye and ear. Current Biology 11, R322-R325. 
Kuhl, P. K., and Meltzoff, A. N. (1982). The bimodal perception of speech in infancy. Science 218, $1138-1141$.

Kujala, T., Karma, K., Ceponiene, R., Belitz, S., Turkkila, P., Tervaniemi, M., and Näätänen, R. (2001). Plastic neural changes and reading improvement caused by audiovisual training in reading-impaired children. Proceedings of the National Academy of Sciences 98, 1050910514.

Liberman, A. M. (1992). The relation of speech to reading and writing. In Orthography, Phonology, Morphology and Meaning, R. Frost, and L. Katz, eds. (Amsterdam, Elsevier Science Publishers B.V.).

Macaluso, E., George, N., Dolan, R., Spence, C., and Driver, J. (2004). Spatial and temporal factors during processing of audiovisual speech: a PET study. Neurolmage In Press.

Martin, A., and Chao, L. L. (2001). Semantic memory and the brain: structure and processes. Current Opinion in Neurobiology $1 /, 194-201$.

Massaro, D. W., and Cohen, M. M. (1993). Perceiving asynchronous bimodal speech in consonantvowel and vowel syllables. Speech Communication 13, 127-134.

Massaro, D. W., Cohen, M. M., and Smeele, P. M. (1996). Perception of asynchronous and conflicting visual and auditory speech. Journal of the Acoustical Society of America 100 , $1777-1786$.

McGurk, H., and MacDonald, J. (1976). Hearing lips and seeing voices. nature 263, 747.

Meredith, M. A., Nemitz, J. W., and Stein, B. E. (1987). Determinants of multisensory integration in superior colliculus neurons. I. Temporal factors. Journal of Neuroscience 7, 3215-3229.

Mesulam, M. (1998). From sensation to cognition. Brain 121, 1013-1052.

Morais, J., and Kolinsky, R. (2005). Literacy and cognitive change. In The science of reading - a handbook, M. Snowling, and C. Hulme, eds. (Oxford, Blackwell Publishing).

Munhall, K., Gribble, P., Sacco, L., and Ward, M. (1996). Temporal constraints on the McGurk effect. Perception \& Psychophysics 58, 35 I-362.

Munhall, K., and Vatikiotis-Bateson, E. (1998). The moving face during speech communication. In Hearing by eye II: advances in the psychology of speechreading and auditory-visual speech, R. Campbell, ed. (Hove, Psychology Press), pp. 123-139.

Munhall, K., and Vatikiotis-Bateson, E. (2004). Spatial and Temporal Constraints on Audiovisual Speech Perception. In The Handbook of Multisensory Processes, G. A. Calvert, C. Spence, and B. E. Stein, eds. (Cambridge, MA, The MIT Press), pp. 177-188.

Olson, I. R., Christopher Gatenby, J., and Gore, J. C. (2002). A comparison of bound and unbound audio-visual information processing in the human cerebral cortex. Cognitive Brain Research 14, 129-138.

Paulesu, E., Perani, D., Blasi, V., Silani, G., Borghese, N. A., De Giovanni, U., Sensolo, S., and Fazio, F. (2003). A Functional-Anatomical Model for Lipreading. Journal of Neurophysiology 90, 2005-2013.

Raij, T., Uutela, K., and Hari, R. (2000). Audiovisual integration of letters in the human brain. Neuron 28, 617-625.

Richards, T. L., Corina, D., Serafini, S., Steury, K., Echelard, D. R., Dager, S. R., Marro, K., Abbott, R. D., Maravilla, K. R., and Berninger, V. W. (2000). Effects of a phonologically driven treatment for dyslexia on lactate levels measured by proton MR spectroscopic imaging. American Journal of Neuroradiology 21, 916-922.

Rockland, K. S., and Ojima, H. (2003). Multisensory convergence in calcarine visual areas in macaque monkey. International Journal of Psychophysiology 50, 19-26.

Schroeder, C. E., and Foxe, J. J. (2002). The timing and laminar profile of converging inputs to multisensory areas of the macaque neocortex. Cognitive Brain Research 14, 187-198.

Schroeder, C. E., and Foxe, J. J. (2005). Multisensory contributions to low-level, 'unisensory' processing. Current Opinion in Neurobiology 15, 1-5. 
Sekiyama, K., Kanno, I., Miura, S., and Sugita, Y. (2003). Auditory-visual speech perception examined by PMRI and PET. Neuroscience Research 47, 277-287.

Shams, L., Kamitani, Y., and Shimojo, S. (2002). Visual illusion induced by sound. Cognitive Brain Research 14, 147-152.

Simos, P. G., Fletcher, J. M., Bergman, E., Breier, J. I., Foorman, B. R., Castillo, E. M., Davis, R. N., Fitzgerald, M., and Papanicolaou, A. C. (2002). Dyslexia-specific brain activation profile becomes normal following successful remedial teaching. Neurology 58, 1203-1213.

Stein, B. E., and Wallace, M. T. (1996). Comparisons of cross-modality integration in midbrain and cortex. Progress in Brain Research 112, 289-299.

Sumby, W. H., and Pollack, I. (1954). Visual contribution to speech intelligibility in noise. The Journal of the Acoustical Society of America 26, 212-215.

Temple, E., Deutsch, G. K., Poldrack, R. A., Miller, S. L., Tallal, P., Merzenich, M. M., and Gabrieli, J. D. (2003). Neural deficits in children with dyslexia ameliorated by behavioral remediation: Evidence from functional MRI. Proceedings of the National Academy of Sciences I00, 28602865.

Van Alteveldt, N., Formisano, E., Goebel, R., and Blomert, L. (2004). Integration of letters and speech sounds in the human brain. Neuron 43, 271-282.

von Kriegstein, K., Kleinschmidt, A., Sterzer, P., and Giraud, A. (2005). Interaction of Face and Voice Areas during Speaker Recognition. Journal of Cognitive Neuroscience 17, 367-376.

Wallace, M. T., Wilkinson, L. K., and Stein, B. E. (1996). Representation and integration of multiple sensory inputs in primate superior colliculus. Journal of Neurophysiology 76, 1246-1266.

Welch, R. B., and Warren, D. H. (1980). Immediate perceptual response to intersensory discrepancy. Psychological Bulletin 88, 638-667.

Wright, T. M., Pelphrey, K. A., Allison, T., McKeown, M. J., and McCarthy, G. (2003). Polysensory Interactions along Lateral Temporal Regions Evoked by Audiovisual Speech. Cerebral Cortex 13, 1034-1043. 


\section{SAMENVATting}

In dit proefschrift wordt onderzocht hoe letters en spraakklanken in de hersenen van volwassenen die goed kunnen lezen, met elkaar geassocieerd worden. Het eerste doel is om het inzicht in de neurale basis van alfabetisch lezen te vergroten, het tweede om bij te dragen aan de fundamentele kennis over hoe de hersenen informatie uit verschillende zintuigen verenigen (cross-modale of multisensorische integratie). We hebben gebruik gemaakt van de neuro-imaging techniek fMRI (functional Magnetic Resonance lmaging), waarmee het mogelijk is met hoge ruimtelijke nauwkeurigheid dynamische hersenprocessen tijdens sensorische of cognitieve activiteiten in kaart te brengen.

\section{De relatie tussen spraak en schrift}

Men schat dat spreken ongeveer honderdduizend jaar oud is, terwij! schrijven pas zo'n vijfduizend jaar bestaat en het daarna nog een flinke tijd heeft geduurd voordat lezen en schrijven gemeengoed werden. Gesproken taal heeft zich dus veel langzamer en natuurlijker kunnen ontwikkelen; het is een evolutionair ontwikkelde hersenfunctie. Geschreven taal daarentegen is een culturele uitvinding: onze hersenen zijn niet door de evolutie gevormd om te kunnen lezen en schrijven.

We vinden het leeftijdsverschil tussen spraak en schrift terug in onze persoonlijke ontwikkeling. We leren gesproken taal vanaf onze geboorte automatisch aan, terwijl lezen en schrijven expliciete instructie vereisen: we moeten leren hoe we de al verworven gesproken taal kunnen weergeven door schrift. In op spraak gebaseerde alfabetische schriften, zoals het Nederlands en het Engels, wordt gesproken taal weergegeven op het niveau van betekenisloze spraakeenheden: de letters van het alfabet staan elk voor een losse spraakklank. Denk aan het woord "vis" (uit het rijtje boom-roos-vis): de letters " $v$ ", " $i$ ", en "s" geven de klanken /v/, /i/ en /s/ weer. Letters en klanken zijn daarom de meest elementaire eenheden waarop spraak en schrift met elkaar overeenkomen en zij vormen zo de basis van lezen in alfabetische schriften. Leren lezen is dus eigenlijk een cross-modale taak: auditieve spraakeenheden moeten geassocieerd worden met visuele symbolen (letters). Het aanleren en automatiseren van letter-klankkoppelingen blijkt vooral erg moeilijk voor kinderen met ontwikkelingsdyslexie.

\section{De vereniging van de zintuigen}

Onze zintuigen lijken losse apparaten die onafhankelijk van elkaar hun werk doen. We zien met onze ogen, horen met onze oren en ruiken met onze neus. Ook in de hersenen lijkt de verschillende informatie in aparte gebieden verwerkt te worden. Toch wordt wat we zien ook weer verenigd met wat we horen: alleen dán nemen we alles in de omgeving als kloppend geheel waar. Dit wordt cross-modale of multisensorische integratie genoemd. Deze eigenschap van de hersenen maakt het mogelijk om letters aan klanken, en zo schrift aan spraak, te koppelen.

Schrift vormt een kunstmatige visuele component van spraak; het is niet natuurlijk ontstaan maar door de mens uitgevonden. Naast schrift heeft spraak ook een natuurlijke 
visuele component: de lipbewegingen van de spreker. Liplezen tijdens luisteren naar spraak wordt audiovisuele spraakwaarneming genoemd en de neurale basis hiervan is uitvoerig onderzocht in neuro-imaging studies. Calvert en collega's stelden aan de hand van fMRI-experimenten een model op waarbij auditieve en visuele spraak geïntegreerd wordt in de superieure temporale sulcus (STS). De verwerking in sensorische hersengebieden, zoals de auditieve cortex, wordt vervolgens beïnvloed door feedbackprojecties vanuit de STS. Over het neurale mechanisme waarmee spraak en schrift geïntegreerd worden, is nog maar weinig bekend.

Een belangrijke vraag bij cross-modale integratie is hoe de hersenen weten welke beelden bij welke geluiden horen. Er zijn diverse factoren gevonden die een rol spelen in dit proces: bindingsfactoren. Zo worden twee stimuli eerder aan elkaar gekoppeld als ze tegelijk optreden (temporele relatie), uit dezelfde locatie komen (spatiële relatie), of overeenkomen in betekenis of identiteit (betekeniscongruentie). Naast deze stimulus-gerelateerde bindingsfactoren kan cross-modale integratie ook beïnvloed worden door de vereisten van een bepaalde experimentele taak. In dit proefschrift zijn de rol van betekeniscongruentie (hoofdstuk $2,3 \& 4$ ), temporele relatie (hoofdstuk 3 ) en taakfactoren (hoofdstuk 4) op de integratie van letters en spraakklanken in de hersenen onderzocht.

\section{De experimenten}

Hoofdstuk 2 doet verslag van een studie waarin we de betekeniscongruentie van letters en klanken hebben gemanipuleerd: proefpersonen kregen zowel congruente (bv. letter " $a$ " en klank /a/) als incongruente (letter "e" en klank /a/) letter-klankcombinaties te zien en horen, terwijl hun hersenen gescand werden. Ook werden de letters en klanken afzonderlijk aangeboden. Een hersengebied in de auditieve cortex dat specifiek op spraakklanken reageert, de planum temporale (PT), bleek sterk te worden beïnvloed door letters: congruente letters leidden tot een verhoogde activiteit, incongruente letters tot een verzwakte. De STS reageerde sterker op de letter-klankparen dan op de afzonderlijke letters en klanken, wat wijst op integratie.

In hoofdstuk 3 de weergave van ons onderzoek naar hoe de temporele relatie de integratie van letters en klanken beïnvloedt. De letter-klankcombinaties werden behalve tegelijk, nu ook aangeboden met een kort interval ertussen (150 of $300 \mathrm{~ms}$ ). Resultaat: de congruentie-effecten in de auditieve cortex treden alleen op als de letterklankparen tegelijk worden aangeboden, terwijl de STS letters en klanken waarschijnlijk over een groter tijdsinterval kan integreren.

In hoofdstuk 4 ons onderzoek naar letter-klank-integratie bij verschillende manieren van stimulusaanbieding en verschillende takkvereisten. In de experimenten van hoofdstuk 2 en 3 werden de stimuli in blokken aangeboden van dezelfde conditie (bv. congruent), in deze studie kwamen ze één voor één in willekeurige volgorde. Terwijl de proefpersonen in de voorafgaande experimenten alleen hoefden te kijken en luisteren naar de letters en de klanken, moesten ze nu tegelijk een taak uitvoeren: beslissen of de letter-klankparen kloppend waren of niet. Dezelfde congruentie-effecten werden gevonden in de auditieve cortex voor de verschillende manieren van stimulusaanbieding. We vonden een tegengesteld resultaat als de proefpersonen de taak moesten uitvoeren: de effecten van congruentie en temporele relatie bleven uit. 


\section{Discussie en conclusies}

Uit de in hoofdstuk 2 en 3 beschreven resultaten hebben we het volgende neurale mechanisme voor de integratie van letters en spraakklanken opgesteld. Spraakklanken worden primair verwerkt in de auditieve cortex (PT). In het volgende verwerkingsniveau, de STS, komt ook visuele informatie over letters binnen. Spraakklanken en letters worden hier geïntegreerd over een groter tijdsinterval. Alleen als letters en klanken tegelijk worden aangeboden, koppelt de STS informatie over de betekeniscongruentie terug naar de auditieve cortex, waar de verwerking van spraakklanken wordt beïnvloed.

De resultaten uit hoofdstuk 4 wijzen erop dat het mechanisme anders werkt als er een actieve taak moet worden uitgevoerd: de auditieve cortex wordt dan niet beïnvloed door de betekeniscongruentie en temporele relatie van de letters en klanken. De taakvereisten lijken de stimulus-gerelateerde bindingsfactoren te domineren: omdat bij elke stimulus een beslissing moet worden genomen zijn allen van gelijke relevantie, onafhankelijk van congruentie of temporele relatie.

Het onderzoek in dit proefschrift beoogde het inzicht te vergroten in de neurale basis van alfabetische lezen. Door de beschreven experimenten weten we nu hoe letterklankkoppelingen worden geïntegreerd in de hersenen van volwassen, ervaren lezers. Tevens hebben we de invloed van verschillende factoren (temporele relatie en taakvereisten) in kaart gebracht. Dit biedt een werkmodel om verder te onderzoeken hoe de letter-klank-integratie in de hersenen zich ontwikkelt in normale lezers en kinderen met ontwikkelingsdyslexie.

Het tweede doel was de fundamentele kennis over cross-modale integratie in de menselijke hersenen te vergroten. Door de onnatuurlijkheid van letter-klankkoppelingen zijn ze een bijzonder geval van cross-modale associaties. Terwijl er al veel onderzoek gedaan is naar natuurlijk gerelateerde cross-modale informatie, zoals audiovisuele spraak, is er nog maar weinig bekend over integratie van artificieel gerelateerde informatie. Uit onze experimenten is gebleken dat het neurale mechanisme voor integratie van letter-klankkoppelingen, en de rol van temporele relatie, overeenkomsten vertoont met de eigenschappen van multisensorische neuronen in verschillende diersoorten. Dit wijst erop dat als letter-klankkoppelingen geleerd en geautomatiseerd zijn, ze geïntegreerd worden door een basaal neuraal mechanisme.

Het in dit proefschrift voorgestelde mechanisme voor de integratie van letters en spraakklanken lijkt sterk op het in de literatuur voorgestelde mechanisme voor audiovisuele spraakwaarneming. Dit ondersteunt het interessante idee dat het natuurlijke, evolutionair ontwikkelde hersenmechanisme voor audiovisuele spraakintegratie een neurale basis zou kunnen bieden voor het vormen van de kunstmatige koppeling van spraak aan schrift. Dit zou een verklaring kunnen bieden voor waarom de meeste mensen zo efficiënt kunnen lezen, terwijl het zo'n onnatuurlijke taak is. 


\section{ACKNOWLEDGEMENTS}

More than five years ago, I packed my things and moved from Utrecht to Maastricht. Looking back, it has been a major part of my life so far, of which the end result is not only this book. I have met and worked together with a lot of inspiring people, made new friends, and fortunately also kept old friends. Both scientifically and personally it has been a great time, to which many people contributed.

First of all, I am very grateful to my "team" of supervisors: Leo Blomert, Elia Formisano, and Rainer Goebel. You were not only a perfect mix of expertise, but also of enthusiasm, inspiration, and realism. I'm very much looking forward to work with you a little longer!

Many other (ex-) colleagues made life in- and outside the university very enjoyable, through conversations, lunches, parties and musical happenings: Alard, Alex, Amanda, Anke, Bernadette, Bettina, Elia, Federico, Francesco, Henk (J), Holger, Ingrid, Jessica, Joël, Judith, Lourens, Mart, Michael, Milene, Nadia, Peter (V), Peter (de W), Sandra, Vera, Vincent, Wilfred and all others.

Amanda, collega, huisgenoot maar bovenal vriendin, we hebben samen veel gelachen en gegriend. Hopelijk kennen we elkaar nog als we twee oude vrouwtjes zijn. Milene, je bent een hele fijne vriendin. Het was goed je al die tijd zo dicht in de buurt te hebben. Sandra, Jut (of Jul?), ik mis je nog steeds in Maastricht! Gelukkig is de afstand tussen ons alleen praktisch gezien groter geworden. Jessica en Vera, mijn gezellige en lieve kamergenoten. Jessica, jou moeten we helaas binnenkort weer missen. Veel plezier met je nieuwe baan. Vera, ik heb zin in onze samenwerking, en natuurlijk ook in onze wijntjes en reisjes. Holger, bedankt voor je behulpzaamheid en je onweerstaanbare chocolademousse. Michael, jij weet altijd precies wat je moet zeggen om me goed te laten voelen over mezelf. Ik hoop dat we nog veel zullen kletsen, fietsen en biertjes drinken. Peter V, bedankt voor de druiven. En dat je me af en toe transcendeerde naar jouw niveau van denken over wetenschap, inclusief mijn eigen onderzoek.

Alex Sack en Peter de Weerd wil ik nog extra bedanken voor de grondige feedback op eerdere versies van hoofdstuk 1 en 5. Mijn "schrijfgroepje" met Han Beijersbergen in het bijzonder, en Dimitri Tokmetzis wil ik bedanken voor het nuttige commentaar op de Nederlandse samenvatting. Annemie, bedankt voor je geweldige hulp bij alle "regeldingen".

During the last few years, I was also fortunate to experience science internationally. Many thanks go to Amir Amedi, Michael Beauchamp, Katharina von Kriegstein and Marcus Naumer for working together in the symposium at IMRF 2004 and writing the review paper together that is the basis of chapter 5. I'm looking forward to future collaborations! Furthermore, I would like to thank BJ Casey for the hospitality and Bruce McCandliss for the interesting discussions during my visit at the Sackler 
Institute in New York in 2002. My time at the Sackler has definitely contributed to the ideas that formed the basis of this thesis.

En buiten de wetenschap was er gelukkig ook nog leven in Maastricht. Fraukje, biologie in Utrecht, "iets anders" in Maastricht, guinness in Dublin, shisha's in Istanbul... Wat wordt onze volgende bestemming? Maastricht zou niet hetzelfde zijn geweest als ik niet zo vaak zo hard had kunnen lachen met jou. Madelief, bedankt voor alle gezelligheid. Mijn mede drama- en dancing queens, Annie, Loes en Joel, met jullie heb ik me de afgelopen jaren goed uit kunnen leven als het nodig was.

De vrienden die ik heb "achtergelaten". Machteld, nóg een gezegde is onwaar gebleken: "uit het oog, ...". Astrid, Machteld, Majorie, Suzan: De Wijf beleeft misschien wat minder avonturen, maar zijn zeker nog bij elkaar! En daar ben ik ontzettend blij mee. Ik kom écht nog wel een keer terug...

Papa en mama, jullie aanmoediging heeft zeker een rol gespeeld in mijn keuze naar Maastricht te gaan. Bedankt voor alle interesse en het heerlijke (werk-) plekje op Texel waar ik altijd terecht kan. Bas, ik ben blij dat jij mijn grote broer bent. Wouter, broertje, mede-wetenschapper, ik hoop dat we nog menige flesjes wijn samen legen en dat de van Atteveldt \& van Atteveldt er ooit van komt.

Lieve Dimitri, wat ben ik blij met jou. De laatste loodjes waren zoveel zwaarder geweest zonder jouw interesse en vertrouwen, als jij me niet op de goede momenten aan het lachen had gemaakt, warm had gehouden, en ik dat ideale plekje bij je schouder niet had gehad. 


\section{Curriculum Vitae}

Nienke van Atteveldt was born on September $23^{\text {rd }} 1977$ in IJsselstein, The Netherlands. She attended the O.S.G. De Hogeberg in Den Burg (Texel) and obtained her V.W.O. diploma in 1995. In 1996 she obtained her propadeutic degree in Biology at Utrecht University. From 1996 until 2000 she studied Fundamental Biomedical Sciences at the Faculty of Biology, Utrecht University and graduated in August, 2000. In this period, she specialized in neurosciences and performed two research traineeships. In the first, she investigated the effect of divided attention on visual motion detection in a series of psychophysical experiments, under supervision of Prof. Dr. F. Verstraten (Functional Neurobiology, Utrecht University, 1998-1999). In her second traineeship, she investigated top-down modulation of sensory brain areas during bimodal selective- and divided attention in schizophrenia using fMRI, under supervision of Dr. N. Ramsey (Dept. of Psychiatry, University Medical Center Utrecht, 1999-2000). From 2000 until 2006 she was Ph.D.-student and junior lecturer at the department of Cognitive Neuroscience, Faculty of Psychology, Maastricht University. During this period, she conducted the experiments described in this thesis at the F.C. Donders Centre for Cognitive Neuroimaging (Nijmegen) and the Maastricht Brain Imaging Center (MBIC, Maastricht University), under supervision of Dr. L. Blomert, Dr. E. Formisano and Prof. Dr. R. Goebel. She will continue her work at the department of Cognitive Neuroscience as a post-doc researcher. 